\title{
The EfFect of Particle Surface Area to Volume Ratio on ION RELEASE FROM COCR SPHERES
}

\author{
A Thesis \\ presented to \\ the Faculty of California Polytechnic State University, \\ San Luis Obispo
}

\author{
In Partial Fulfillment \\ of the Requirements for the Degree
}

Master of Science in Engineering, with Specialization in Materials Engineering

by

Darin Joseph Grandfield

June 2009 
(C) 2009

Darin Joseph Grandfield

ALL RIGHTS RESERVED 


\section{COMMITTEE MEMBERSHIP}

TITLE:

AUTHOR:

DATE SUBMITTED:

COMMITTEE CHAIR:

COMMITTEE MEMBER: Paul Rainey, Ph.D

Faculty, Materials Engineering

California Polytechnic State University, San Luis Obispo

COMMITTEE MEMBER: Lanny Griffin, Ph.D

Chair, Biomedical Engineering

California Polytechnic State University, San Luis Obispo 


\section{ABSTRACT}

The EfFect of Particle Surface Area to Volume Ratio on Ion Release from COCR SPHERES

\section{Darin Grandfield}

In 2005, over 200,000 Americans underwent a hip arthroplasty, the replacement of a hip joint with an artificial prosthesis. Of these arthroplasties, metal-on-metal type implants represent an increasing usage percentage. Metal-on-metal implants are selected largely for their low volumetric wear rate, durability, and resistance to corrosion. In spite of these advantages, little is known concerning the long-term consequences of heavy metal alloy use in the body, although early research indicates potentially carcinogenic results. This thesis is a preliminary investigation into these long term effects and their root causes.

An improved comprehension of the corrosion kinetics and the rate of ion production from the high surface energy wear debris released by implant articulation can assist in illustrating the relative clinical significance of exposure to these metallic bodies over time. This thesis primarily focuses on developing a test methodology for the detection and analysis of ion dissociation in simulated body fluids. In order to validate this test methodology, the ion dissociation rates and surface characteristics of several predetermined diameters of cobalt chromium alloy spherical particles were analyzed.

The effect of changing particle diameter, and thus surface area to volume ratio, on ion dissociation rate was determined to be significant when not affected by localized agglomeration. Additionally, preferential corrosion of cobalt within individual grains was observed and correlated to elevated cobalt concentrations in the electrolyte. These results suggest that ion dissociation kinetics for true wear particles can be determined through the refinement and application of the methodology developed.

Key Words: CoCrMo, CoCr, Cobalt Chromium Alloy, Orthopaedic Implant, Arthroplasty, Corrosion, Ion Dissociation, Kinetics. 


\section{ACKNOWLEDGMENTS}

- Trevor Harding for his endless patience, wealth of knowledge, and continuous support.

- The Materials Engineering Department, Cal Poly SLO for providing equipment and space to work.

- $\quad$ Pat Campbell for her expertise, advice, and supplies.

- Materials Research Facilities Network (MRFN) program at the University of California, Santa Barbara, funded by National Science Foundation grant DMR0520415, for providing instrumentation support used to obtain materials characterization of samples.

- Anika Odukale for her assistance with grants and the facilities at UCSB.

- Joe Doyle for his selfless sharing of equipment at UCSB.

- Dr. Paul Rainey and Dr. Lanny Griffin for being part of the defense committee. 


\section{TABLE OF CONTENTS}

LIST OF TABLES........................................................ viii

LIST OF FIGURES........................................................... ix

\section{CHAPTER}

1. INTRODUCTION .......................................................

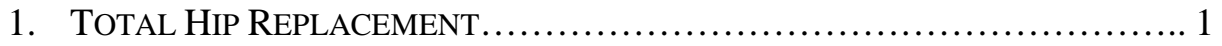

2. LIMITATIONS OF THR........................................... 2

3. COMPARISON OF PRIMARY IMPLANT DESIGNS........................... 4

4. Limitations of THE Metal-ON-PolyethylENE DeSign................ 5

5. Alternative IMPLANT DESIGN: The MoM ARTICULATION ............. 7

6. WEAR DEBRIS GENERATION IN MOM IMPLANTS.........................9

7. Systemic Wear Debris Distribution............................ 12

8. CORROSION OF SYSTEMICALLY DISPERSED COBALT CHROMIUM ALLOY DEBRIS...................................................... 14

9. CONSEQUENCES OF WeAR Debris AND ReSUlting METALLiC IONS......16

10. RESEARCH STATEMENT.............................................. 19

2. MATERIALS AND METHODS......................................... 20

1. Test Plan Overview........................................... 20

2. Materials ........................................................ 20

3. SAMPle PreParation............................................. 22

4. Test Methodology............................................... 23

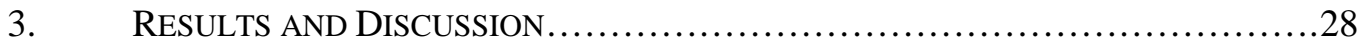

1. OVERVIEW OF OBJECTIVES....................................... 28

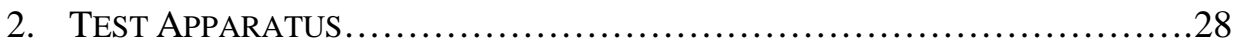

3. DISCUSSION OF EXPERIMENTAL METHODOLOGY IMPROVEMENTS........ 30

4. DATA ANALYSIS AND ION RELEASE RATE FOR INITIAL FOUR VALIDATION STUDIES............................................32

1.Experiment 1: Initial Exploratory study of methodology......... 33

2.Experiment 2: Proving detectable corrosion.................. 35

3. Experiment 3: Low statistical power, broad range of conditions.. 40

4. Experiment 4: Extended duration with higher statistical power... 44

5. EXPERIMENT 5: HIGH STATISTICAL POWER WITH INCREASED LEVELS..... 48 
1. Experiment 5 Results...................................... 49

2.Experiment 5 Discussion ................................ 55

6. Evaluating Surface Morphologies..............................66

4. CONCLUSIONS....................................................... 70

1. OVERVIEW OF OBJECTIVES .................................... 70

2. OBJECTIVE 1: APPARATUS CONSTRUCTION........................... 70

3. OBJeCtive 2: EXPERIMENTAL METHOdOLOGY..........................71

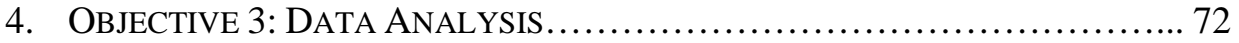

5. Correlation between Surface Area to Volume Ratio AND

DISSOCIATION RATE............................................. 72

1. Cobalt Dissolution...................................... 73

2.Chromium Dissolution.................................... 73

3. Discussion of $\mathrm{pH}$ Modifications............................... 74

6. Concluding StATEMEnT.............................................74

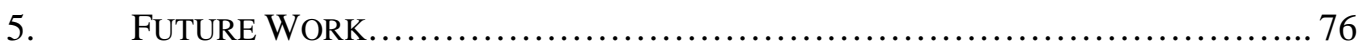

6. WORKS CITED ....................................................... 80 


\section{LIST OF TABLES}

\section{TABLE}

2.1. Comparison between as received samples and ASTM F75 standard for $\mathrm{CoCr}$.......... 21

3.1. Experiment 1 results, illustrating a gradual accumulation or memory effect within the AAS, particularly noticeable in Cr samples. The samples highlighted orange were above $0.07 \mathrm{ppm}$, and the highest detected concentration was highlighted red

3.2. Composition of corroded and uncorroded $400 \mu \mathrm{m}$ diameter spheres as compared to

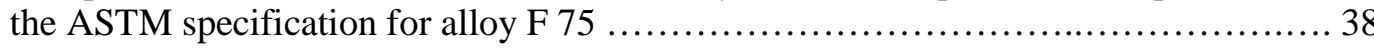

3.3. Tabled results from the third experiment. Results coinciding with the hypothesis of higher dissociation rates for smaller diameter debris are highlighted in red.

Absorptivity measurements were used because only comparisons between samples were made and concentration calculations were unnecessary and introduce error .......442

3.4. Physical features of simulated $\mathrm{CoCr}$ wear debris used in this experiment .............. 56 


\section{LIST OF FIGURES}

\section{FIGURE}

1.1. A healthy biological hip is displayed on the left, showing the interface between the acetabular cup and the femoral head. The hip on the right is an example of

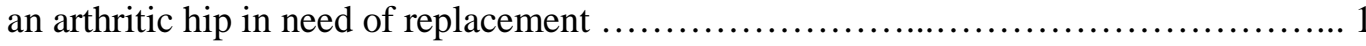

1.2. Radiograph of an implanted THR, illustrating the placement of the implant in relation to the original hip joint

1.3. A contrast between MoM (left) and MoP (right) implants. The clear distinction being the polyethylene liner between the acetabular shell and femoral head on the MoP design

1.4. Resultant forces on the hip during unilateral weight bearing $\ldots \ldots \ldots \ldots \ldots \ldots \ldots \ldots \ldots . \ldots \ldots$

1.5. Polyethylene wear debris from articulation against a $\mathrm{CoCr}$ femoral head. A high percentage of light-colored fibril type debris can be seen

1.6. A) Typical calvarial histology died in toluidine blue. Image displays scar tissue 7 days after implant surgery on a mouse skull. B) Granulation tissue overlying the calvarium 7 days after surgery and polyethylene particle implantation. Marked bone resorption can clearly be identified and key osteolysis locations have been labeled

1.7. Representation of volumetric wear for $\mathrm{CoCr}$ alloy metal on metal implants. Note the initial wear in period followed by steady state wear debris production $\ldots \ldots \ldots \ldots \ldots \ldots .7$

1.8. The principle advantages of the larger femoral head diameter allowed by the elimination of the PE liner include a longer travel distance for the user's femur and a greater contact diameter, which allows for lessened pressure on the femoral head component and a lower resultant wear rate

1.9. Transmission Electron Microscope (TEM) photomicrographs of wear debris generated by hip simulator testing for 2 million cycles. (1) Chromium oxide particle from passivation layer, (2) enriched chromium carbides, and (3) bulk alloy matrix wear debris

1.10. Illustration of microscopic rendering of asperity interaction ath the head-cup interface

1.11. A scratch defect on the surface of a CoCr femoral head during articulation. EDS inset shows foreign particle on edge to be Al2O3. Mag: 1000x

2.1. $40 \mu \mathrm{m}$ (left) and $400 \mu \mathrm{m}$ (right) diameter CoCr spheres used for testing. Note the cast dendritic structure, geometrically spherical shape, and similarity between samples 
2.2. Blending the red colored HBSS with $0.1 \mathrm{M} \mathrm{HCl}$ resulted in a yellow colored $\mathrm{pH} 3$

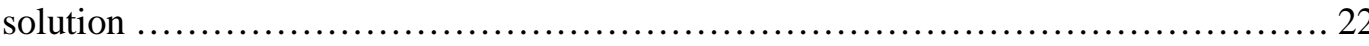

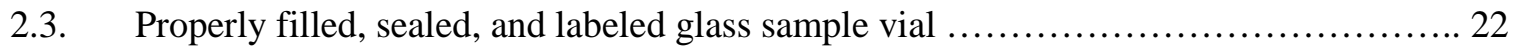

2.4. HDPE sample vials placed at random in the sample holder prior to compression fastening. The four vertical threaded rods allowed for the use of nuts to compress the samples, effectively holding them in place

2.5. New Brunswick G76D incubating shaker. The samples were loaded into the sample holder and attached to the four agitation arms for testing (Image courtesy of New Brunswick)

2.6. Base level of fabricated sample holder. Samples were laid horizontally and secured through compression of aluminum plates above. Flow holes were drilled to allow for adequate distribution of the incubating bath water ................................ 24

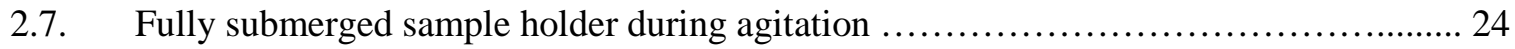

2.8. Vacuum filtration system used to separate debris from electrolyte post test. The Millipore filtration system is on the right

2.9. The millipore system used for particle filtration. The electrolyte was drawn through the filter paper into the flask by a pressure differential ............................. 25

2.10. $40 \mu \mathrm{m}$ diameter debris caught in the $0.2 \mathrm{um}$ filter paper used in the experiments ....... 26

3.1. The New Brunswick G76D Gyratory Shaker Bath, used to repeatably agitate samples at body temperature. Note the 4 arms extending into the bath cavity, to which a sample holder was attached

3.2. In order to validate the uniform heating capacity of the G76D water bath, two thermocouples were placed in the bath. One was placed on the bottom, immediately proximal to the heating element, and the other was placed at the top of the water

3.3. The cobalt ion concentrations detected in the $\mathrm{HCl}$ electrolyte after only 1 day were statistically significant

3.4. Uncorroded CoCr spheres, showing a cast, dendritic structure (A \& B), as compared to the same spheres after 1 day of immersion in $\mathrm{HCl}(\mathrm{C} \& \mathrm{D})$. Clear preferential corrosion can be identified well below the surface of the sphere

3.5. EDS compositional mapping of the surface of a sphere corroded in $\mathrm{HCl}$. In the left image (A), red pixels represent a location of predominantly chromium and green pixels highlight cobalt. In this case, chromium and cobalt are approximately equal, confirming the compositional results that $\mathrm{HCl}$ immersion led to a significant drop in cobalt content, implying preferential cobalt corrosion. Additionally, the right image (B) includes blue pixels, which represent oxygen. The relatively high surface oxygen concentrations led to further investigation into oxide formation during corrosion 
3.6. (A) EDS elemental mapping of exclusively cobalt on the surface of an uncorroded sample. Note the clarity of grain boundary definition. (B) EDS elemental mapping of chromium on the same image with the same contrast. The definition of the grain boundaries is much less clear, indicating a generally higher $\mathrm{Cr}$ concentration around the boundaries

3.7. EDS rendering of a composite map, illustrating regions with the highest $\mathrm{Cr}$ content as those lightest in color

3.8. Cobalt molar absorptivity results as detected by the AAS. A trend towards elevated dissociation rates at lower $\mathrm{pH}$ was observed

3.9. Cobalt molar absorptivity results as detected by the AAS. A trend towards elevated dissociation rates at higher mass loadings was observed

3.10. Cobalt molar absorptivity results as detected by the AAS. A trend towards elevated dissociation rates at smaller particle diameters was observed

3.11. Graphical results from experiment 4. A statistically significant difference was detected between the diameters of the spheres tested as well as the comparative dissociation rates of cobalt and chromium for each diameter level ... 45

3.12. Representation of the effects of electrostatic forces on debris of decreasing diameter ... 47

3.13. A representation of the effect of agglomeration (red line) on the expected particle corrosion rate (blue line). As debris reaches a critical size with a critical interparticle spacing, (which is related to the particle density in solution) the Van der Wals and other electrostatic forces between particles gain significance until they agglomerate. This agglomeration leads to a reduction in total exposed surface area and surface energy, which directly decreases the corrosion rate

3.14. Detected cobalt concentrations at each mass loading level and for each sphere diameter. The significant trend of increasing concentration in relation to both increasing surface area to volume ratio and increasing mass loading can be discerned here.

3.15. Extremely low concentrations of chromium were detected, leading to the conclusion that negligible chromium dissolution occurred during the test period, as all results were below the theoretical detection limit

3.16. An interactions analysis of $\mathrm{Co}$ and $\mathrm{Cr}$ concentrations at each mass loading and sphere diameter level for $\mathrm{pH} 7$ samples. No significant interactions were detected. The potential interaction seen in the $\mathrm{Cr}$ graph was determined to be negligible

3.17. Co ion concentrations detected at the $\mathrm{pH} 3$ level. Potential agglomeration effects can be seen in the decreased ion concentrations at the $40 \mu \mathrm{m}$ level

3.18. Cr ion concentrations detected at the $\mathrm{pH} 3$ level. Potential agglomeration effects can be seen in the decreased ion concentrations at the $40 \mu \mathrm{m}$ level 
3.19. An interactions analysis of $\mathrm{Co}$ and $\mathrm{Cr}$ concentrations at each mass loading, $\mathrm{pH}$, and sphere diameter level. Strong evidence of an interaction exists between $\mathrm{pH}$ and sphere diameter as well as between mass loading and sphere diameter at $\mathrm{pH} 3$

3.20. The dendritic structure of the spherical ASTM F75 CoCr alloy particles used to simulate wear debris

3.21. Estimated dissociation mass (ug) for micro-scale wear debris. Browne's linear fit curve (blue line) was extrapolated to $0.005 \times \mathrm{E} 10 \mu \mathrm{m} 2$ (red line) in order to include the large-diameter samples with low total exposed surface areas used in this experiment

3.22. For particulate debris samples, the theoretical surface area to volume ratio rises dramatically at particle diameters below $50 \mu \mathrm{m}$. This represents an increase in the relevance of the surface area term

3.23. The predicted ion concentrations for $400 \mu \mathrm{m}$ and $40 \mu \mathrm{m}$ particles compared to the actual results

3.24. Plot of results of experiment 5 and the fit curve used to extrapolate data to true wear debris, based on knowledge of the significance of the surface area term in the surface area to volume ratio at particle diameters below $40 \mu \mathrm{m}$

3.25. The power fit curve used to incorporate the data from this experiment (blue points), work by Browne et al. (orange points), and an extrapolation (red line) to true wear debris dimensions (red points)

3.26. In vitro serum concentrations of Co in a wear and dissociation study. Serum ion levels were shown to become relatively saturated after a 24 hour period

3.27. Bar chart of clinical and empirical results for particulate dissociation rates .............66 66

3.28. Mild corrosion effects were visible at close magnifications when comparing uncorroded samples (top) to samples corroded in $\mathrm{pH} 7$ for 50 days

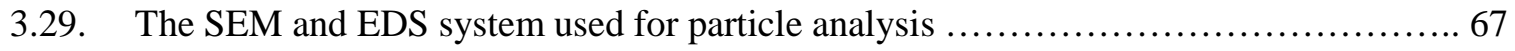

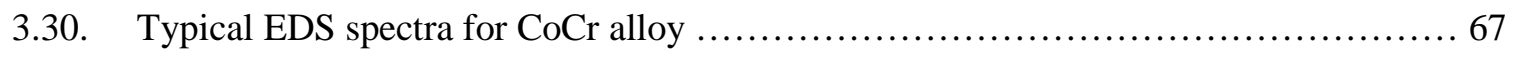

3.31. FIB cross sections of the CoCr spheres. Cross sections were performed with the intention of characterising a distinctive oxide layer depth

3.32. Backscatter detection of the FIB cross section seen in figure 4.26. The lighter region near the sample surface potentially indicates a compositional difference and possible oxide layer

4.1. Extrapolation of exponential fit curve for empirical data to true wear debris diameters. The blue points indicate experimental data from this experiment, while the red data point is a suggested dissociation rate for true wear debris of $400 \mathrm{~nm}$ diameter. This extrapolation (red point) emphasizes the exponential relationship between total particle surface area to volume ratio and decreasing particle diameter 
5.1. The simplified procedure (blue) used for this series of tests and the addition of necessary steps and improvements (red) for the application of this test methodology

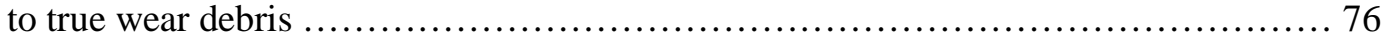

5.2. Photomicrograph of synovial tissue with large amount of particulate metal, giant cells, and reactive fibrosis. (Hematoxylin-eosin stain,) (original magnification $\mathrm{x} 400$ ) 



\section{INTRODUCTION}

\subsection{TOTAL HIP REPLACEMENT}

Total hip replacement (THR) is one of the most effective orthopedic procedures performed today. Also referred to as hip arthroplasty, THR is a surgically invasive procedure during which one or both of the patient's biological hip joints (Figure 1.1) are replaced with near fully

functional artificial prosthesis'.

The Center for Disease Control

and Prevention reported that

235,000 total hip replacements
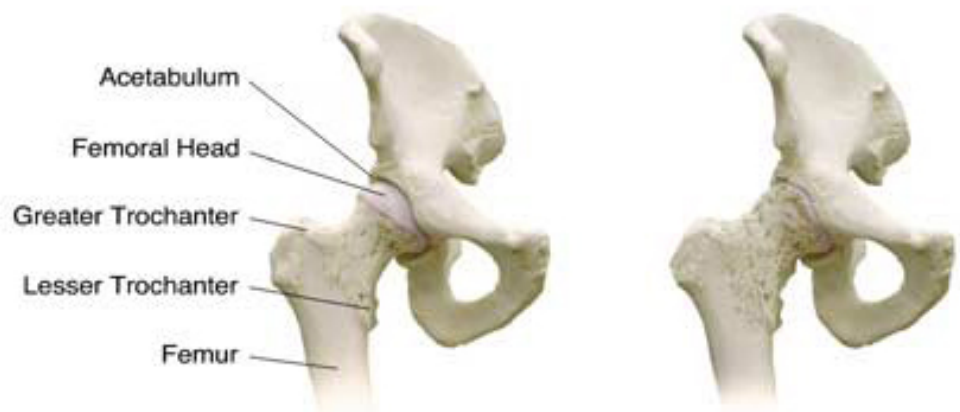

were performed in the U.S. in 2005

(DeFrances \& Hall, 2007). This

Figure 1.1: A healthy biological hip is displayed on the left, showing the interface between the acetabular cup and the femoral head. The hip on the right is an example of an arthritic hip in need of replacement (Hip Anatomy, 2008).

number is rising as implant technology improves and patient recovery time is reduced.

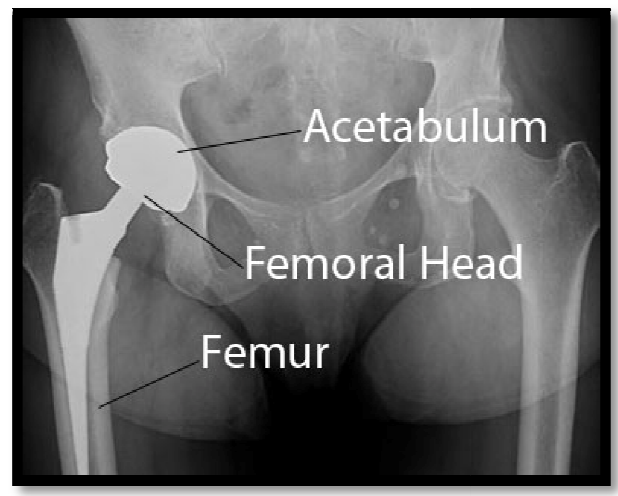

Figure 1.2: Radiograph of an implanted THR, illustrating the placement of the implant in relation to the original hip joint (Dunn, Peters, \& Hofmann, 2000).
The modern hip onsists of individual acetabular and femoral components. The acetabular component is a cup shaped cavity, which is inserted in the human acetabulum. The femoral component consists of two pieces: a ball that replaces the femoral head and a stem, which is inserted into the femur (Figure 1.2). The implantation of the femoral

prosthesis requires the permanent removal of the biological femoral head. There are numerous bearing articulation options for patients undergoing a THR, including metalon-metal (MoM), metal-on-polyethylene (MoP), ceramic-on-ceramic $(\mathrm{CoC})$, and 
ceramic-on-polyethylene (CoP). The two most common prosthesis varieties and those that will be discussed in this document are the MoM and the MoP bearing types (Figure $1.3)$.
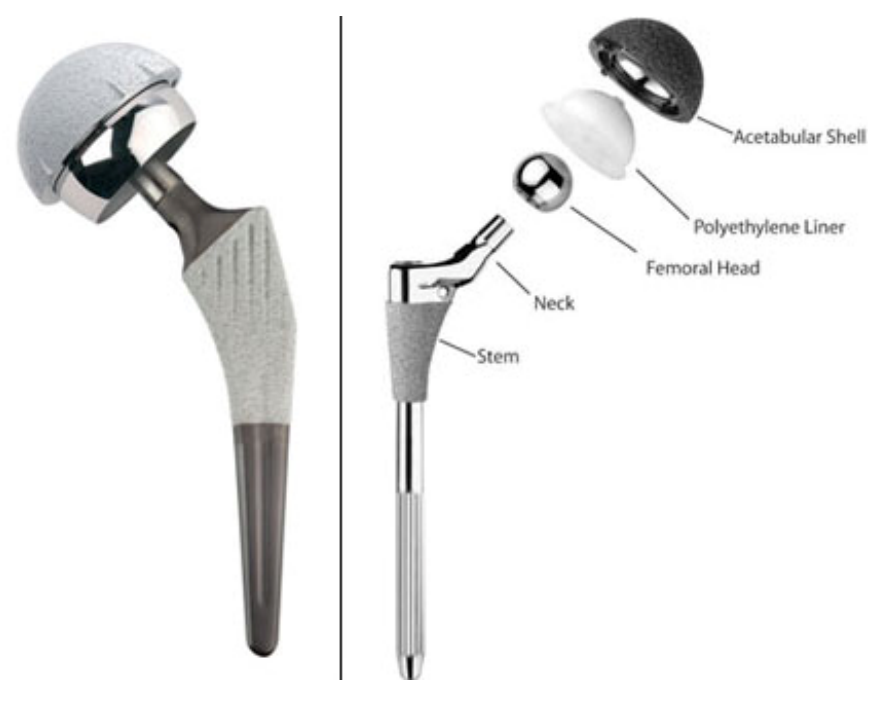

Figure 1.3: A contrast between MoM (left) and MoP (right) implants. The clear distinction being the polyethylene liner between the acetabular shell and femoral head on the MoP design (Direct Healthcare International, 2008).

\subsection{LIMITATIONS OF THR}

While patients of older age are the primary recipients of artificial hip joints, hip

disorders requiring surgery are not restricted to the elderly. There are increasing numbers of young patients that require an artificial hip joint as a result of degenerative joint diseases or injuries. However, according to the Swedish National Hip Arthroplasty Register, the outcomes of THR in patients under the age of fifty are generally not as successful as in older patients (Herberts, Kärrholm, \& Garellick, 2004). Youthful patients are typically far more active than their older counterparts, implying that their joint prosthesis' endure many more cycles per year at the potentially higher loads caused by activities such as running and jumping. Youthful patients also require an extended implant life as they are expected to live longer. 
Due to concerns regarding the long-term implantation of any foreign object in the often hostile environment that is the human body, the safety of orthopaedic implants has been researched in depth for many years. Implant safety can be separated into two key functional areas: (1) mechanical usability and (2) long-term health of the patient. This document will focus exclusively on the long term health implications of prosthetic hips in terms of adverse effects on the natural biological function of the human anatomy. A significant portion of the viability of an implant can be correlated to the materials used for such an implant. The materials used must be biocompatible in a way that both the bulk implant and any associated materials, including released wear debris, do not have significant adverse effects on routine bodily function. Wear is of particular concern in hip prosthetics due to their high loading and frequency of use. In routine activity, the human hip undergoes approximately 1,000,000 cycles annually and is subjected to a maximum of 2.33 times the weight of the human body during loading (Figure 1.4) (Quesnel, Gueritey, \& Gonon, 1995; Carr \& Goswami, 2007).

Historically, the types of materials used in hip prosthetics have varied widely. The modern material standard for a hip arthroplasty is cobalt-chromiummolybdenum alloy $(\mathrm{CoCr})$, which is employed in the

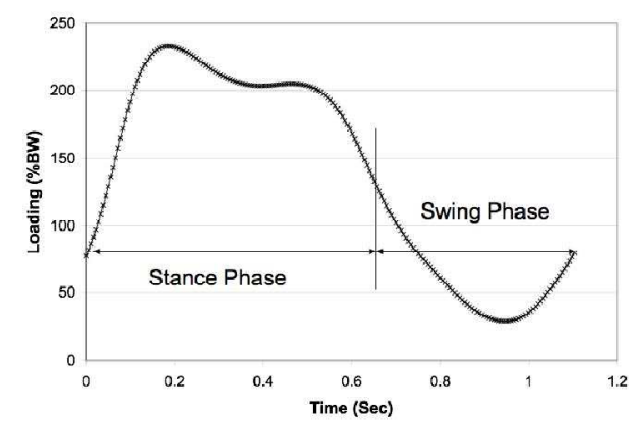

Figure 1.4: Resultant forces on the hip during unilateral weightbearing (Quesnel, Gueritey, \& Gonon, 1995).

femoral head as well as the acetabular cup by both the MoM

and MoP prosthetic designs. The critical difference between the two designs is the ultra high molecular weight polyethylene (UHMWPE) liner found in the acetabular component of the MoP bearing (Figure 1.3). This liner is an effective replacement for the cartilage in 
a biological hip in terms of coefficient of friction and shock absorption, however recent research has shown a correlation between failure of MoP implants and the polyethylene wear debris released during use (Campbell, Amstutz, Kossovsky, \& Clarke, 1992). The MoM design bypasses the issues associated with this liner and utilizes a metal-to-metal interface with a larger femoral head to fill the space occupied by the polyethylene liner.

\subsection{COMPARISON OF PRIMARY IMPLANT DESIGNS}

Sir Charles Charnley's development of the ultra high molecular weight polyethylene (UHMWPE) liner for hip implants in 1962 was heralded as a new era in orthopaedic science (Kurtz, 2004). The UHMWPE acetabular liner was used to replace the function of cartilage in the biological hip joint and operated in conjunction with the extremely hard and biocompatible CoCr alloy (Gray, 1974). The new liner provided both an impact absorbing cushion as well as a tremendously smooth articulating interface that yielded significantly less wear and more biocompatibility than previous implants.

Orthopaedic surgeons quickly embraced Charnley's UHMWPE liner as an alternative to the rapidly wearing polytetraflouroethylene (PTFE). Although it underwent a great deal of development, including polymer cross-linking, the basic metal-on-UHMWPE (MoP) interface remains the dominant implant type used after 45 years (Kurtz, 2004).

A common alternative to MoP is the metal-on-metal (MoM) design. While MoM implants were introduced in the early 1970's, they were not commonly used until recently. Early design flaws and manufacturing difficulties in MoM implants led to rapid failures and low incentive for continued development in the presence of successful MoP designs. Currently, with increasing data concerning wear-related failures of UHMWPE liner implants, MoM designs have been reintroduced as an implant with longer life 
expectancy and potentially higher performance than its MoP counterpart (Campbell, Amstutz, Kossovsky, \& Clarke, 1992). This reintroduction was also a result of the rapidly decreasing age of the average joint replacement patient. Only $8 \%$ of patients undergoing hip replacement procedures were under 60 years of age in 1993 and this number rose to $23 \%$ in 2005 (Chidambaram \& Cobb, 2007). With the ages of hip implant recipients declining, new, longer lasting implants had to be developed that would outlive their users and prevent costly revision surgeries.

\subsection{Limitations of THE Metal-ON-PolyethylENe Design}

The UHMWPE liner implant design continues to be used in implants today, but has gradually lost favor with orthopaedic surgeons, due in part to studies implicating wear induced osteolysis as a primary failure mechanism in MoP implants (Baslé, Bertrand, Guyetant, Chappard, \& Lesourd, 1996). A study by Campbell et al. found that "The clinical consequences of wear debris cover a broad spectrum from radiolucencies to massive osteolysis and implant failure" (Campbell, Amstutz, Kossovsky, \& Clarke, 1992). This and other studies found that the large volume of outsized wear particles (Figure 1.5) released from MoP implants could have significant negative results for the patient, including osteolysis, which is the gradual dissolution and resorption of bone through artificially increased

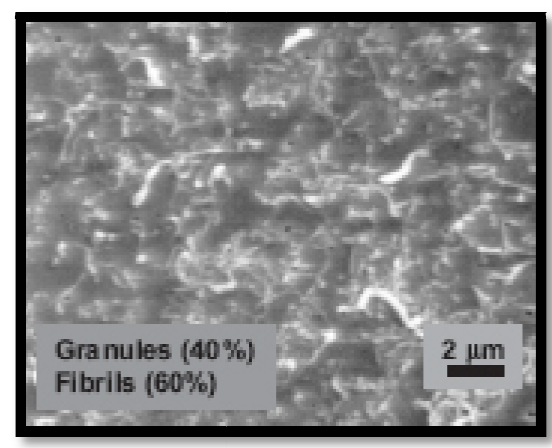

Figure 1.5: Polyethylene wear debris from articulation against a CoCr femoral head. A high percentage of light-colored fibril type debris can be seen (Liaoa, McKellopa, Lua, Campbell, \& Benyaa, 2003).

osteoclast activity. According to a time duration study by Savarino et al. in the year 2000, few MoP implants survived an implantation period in excess of 25 years. Several of Savirono's studies implicated polyethylene wear particles (Figure 1.6) in implant failures. 


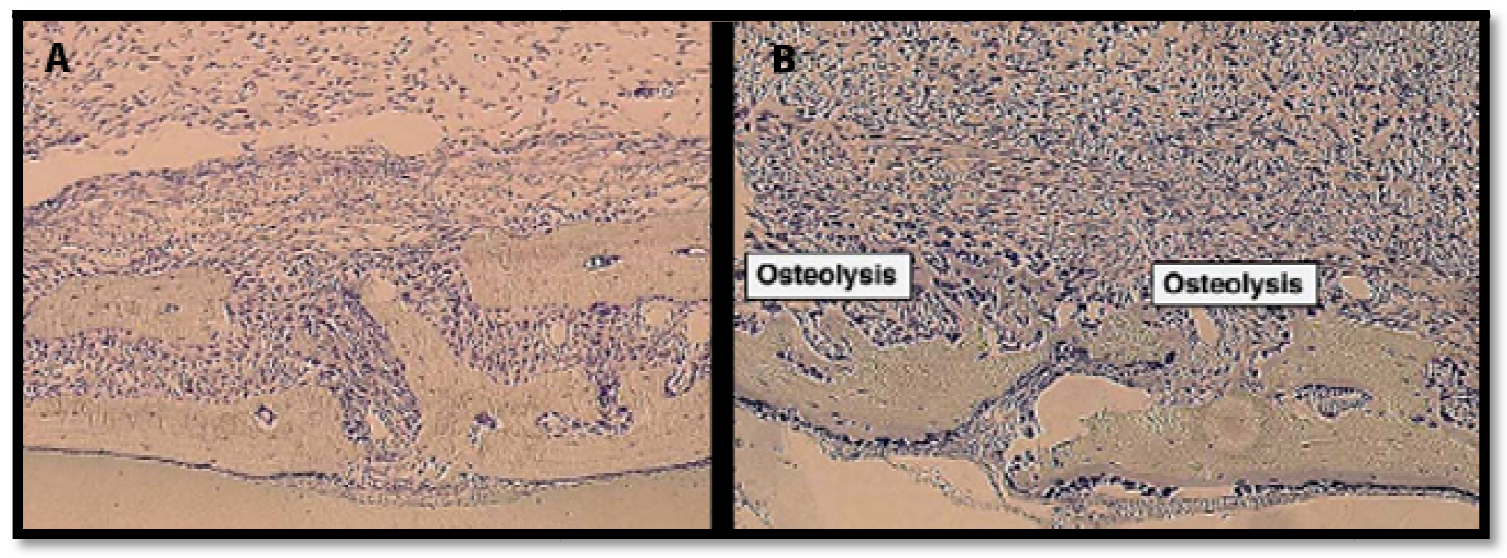

Figure 1.6: A) Typical calvarial histology died in toluidine blue. Image displays scar tissue 7 days after implant surgery on a mouse skull. B) Granulation tissue overlying the calvarium 7 days after surgery and polyethylene particle implantation. Marked bone resorption can clearly be identified and key osteolysis locations have been labeled (Von Knoch, 2004).

These studies claim that polyethylene wear particles lead to a foreign-body reaction with macrophage activation. The inability of human macrophages to digest the inert polyethylene particles leads to release of cytokines and other inflammatory substances, which induce osteoclast production, bone resorption, osteolysis, and eventually the onset of aseptic loosening (Savarino, et al., Ion Release in Patients with Metal-on-Metal Hip Bearings in Total Joint Replacement, 2002). Osteolysis often results in aseptic loosening of implants and ultimately requires a revision surgery, in which the surgeon must operate on significantly less viable bone, which leads to a higher implant failure rate in addition to a more painful implantation for the patient.

Given the high number of daily loading cycles hip prosthetics are subjected to, gradual wear of the bearing surfaces is a primary concern in their design. Both the MoM and MoP bearing systems are designed in order to minimize wear rates. The UHMWPE liner wears at approximately 50x the volumetric rate of the MoM system (Cuckler, 2005), releasing large, chemically inert, wear debris into the periprosthetic tissue at the implant site. Many studies implicate the massive quantity of polyethylene wear debris released by 
MoP implants in several types of failures, particularly aseptic loosening caused by osteolysis.

\subsection{Alternative Implant Design: the Metal-ON-Metal articulation}

The MoM bearing design eliminates the problem of osteolysis related failures

from polyethylene wear debris by entirely removing the UHMWPE liner in favor of a

larger femoral head comprised entirely of cobalt chromium alloy. The potential

advantages of MoM over MoP implants consist of a lower volume of wear particles

generated, lower risk of dislocation, and increased implant longevity (Cuckler, 2005).

The production of wear particles at the acetabular interface in MoM implants is regulated by numerous factors, including surface roughness, nature of the biofilm, and coefficient of friction between the acetabular cup and the femoral head (Hallab, Skipor, \& Jacobs, 2003). The joint fluids present during normal operation of a biological hip continue to lubricate the acetabular interface post operation. This biofilm of joint fluids and proteins formed on the CoCr alloy surface lead to a lower coefficient of friction than MoP interfaces (Lin \& Bumgardner, 2004). While the initial volumetric wear rate of MoM implants is similar to MoP designs, the MoM wear rate reduces to 20 to 100 times less than MoP after a polishing "wear-in" period of 1-2 years (Figure 1.7) (Chan, Bobyn, Medley, Krygier, \& Tanzer, 1999). MoP implants may wear

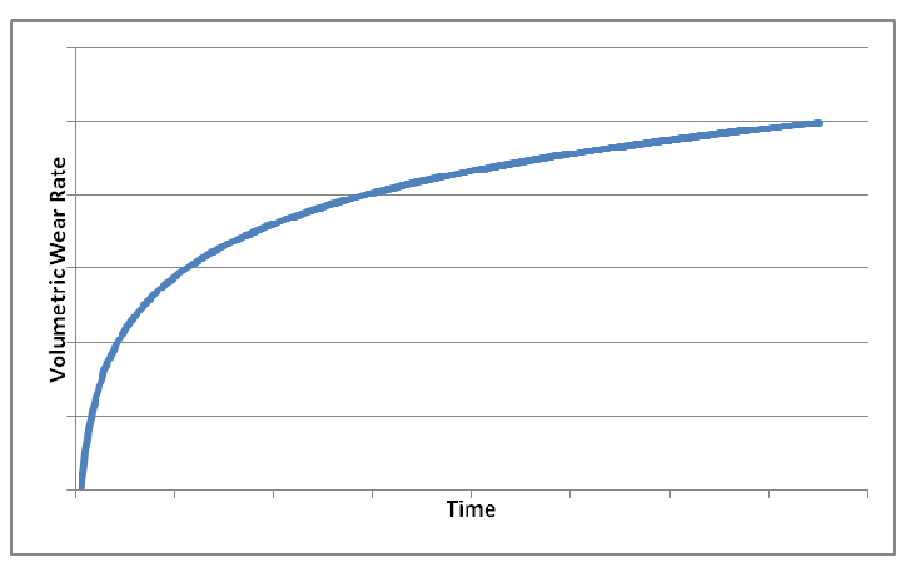

Figure 1.7: Representation of volumetric wear for CoCr alloy metal on metal implants. Note the initial wear in period followed by steady state wear debris production (Dowson, 2006). 
much as 200um/year, while MoM implants typically have a linear wear rate of approximately 5um/year (MacDonald, et al., 2003). It is hypothesized that the lower volume of wear particles released by MoM bearings is directly responsible for a lower risk of aseptic stem loosening than MoP bearings (Naudie, Roeder, Parvizi, Berry, Eggli, \& Busato, 2009). Also, with no polyethylene liner present, the femoral head size can be increased dramatically, further depressing wear. The wear rate is lowered due to a larger bearing surface, which allows for a thicker layer of lubricious joint fluid to fill the microscopic void at the interface (Surin, 2009), as well as lower contact stresses between the acetabulum and the femoral head (Figure1.8).

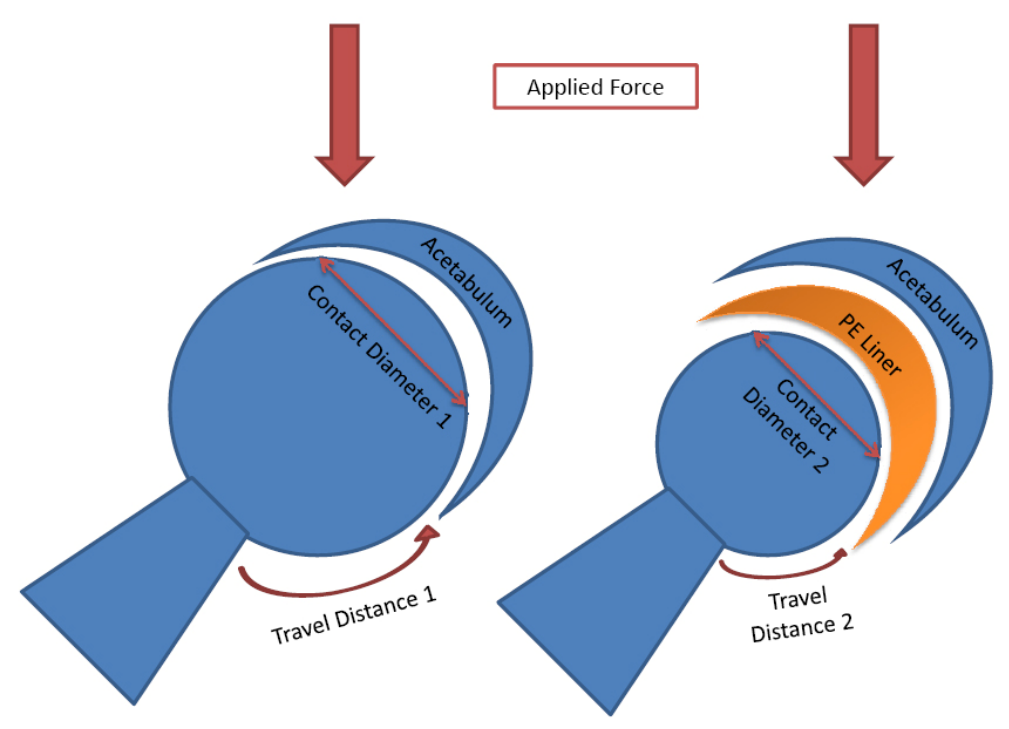

Figure 1.8: The principle advantages of the larger femoral head diameter allowed by the elimination of the PE liner include a longer travel distance for the user's femur and a greater contact diameter, which allows for lessened pressure on the femoral head component and a lower resultant wear rate.

In spite of the advantages of low wear, increased range of motion, and fewer dislocations, metal wear particles released by MoM implants introduce new concerns for the medical community and implant patients. Various studies have found that MoM hip prostheses increase systemic blood serum cobalt and chromium ion concentrations by 3 
to 100 times (Naudie, Roeder, Parvizi, Berry, Eggli, \& Busato, 2009). The long-term effects of these ions are completely unknown, but current concerns include hypersensitivity, toxicological effects, mutagenicity, and carcinogenicity (Dumbleton \& Manley, 2005) (Savarino, et al., 2000). Since the local and systemic biologic response to these ions due to long exposure to the metal implants is unknown, this issue is more critical in younger patients who have greater life expectancy but demand the long term high performance of a MoM implant.

\subsection{WEAR DEBRIS GENERATION IN MOM IMPLANTS}

The variety of wear mechanisms at the MoM interface in implants is responsible for the assortment of wear particle morphologies observed during routine implant use (Liaoa, McKellopa, Lua, Campbell, \& Benyaa, 2003). The seemingly random assortment of MoM wear debris size and shape (Figure 1.9) makes the actual ion production rate

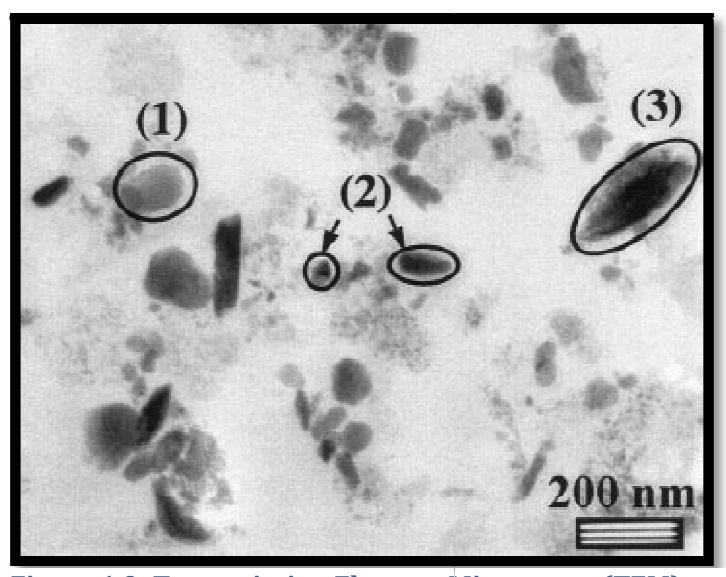

Figure 1.9: Transmission Electron Microscope (TEM) photomicrographs of wear debris generated by hip simulator testing for 2 million cycles. (1) Chromium oxide particle from passivation layer, (2) enriched chromium carbides, and (3) bulk alloy matrix wear debris (Isabelle, Medley, Campbell, Huk, \& Bobyn, 2004). from the implant wear particles difficult to model.

The production and release of potentially toxic metal particles in MoM implants is primarily governed by tribological wear at the acetabular interface. The generation of wear debris that is consequently released into the circulatory,

lymph, and digestive systems is a function of the loading, wear mechanism, and biological factors present at the implant interface. Although there are numerous potential wear mechanisms responsible for the generation of particulate debris in orthopaedic 
implants, the two primary wear mechanisms are abrasive and adhesive wear. Abrasive wear is the interference interaction of asperities, or the nanoscale peaks that represent a true surface at the microscopic level (Figure 1.10), during articulation. Abrasive wear is

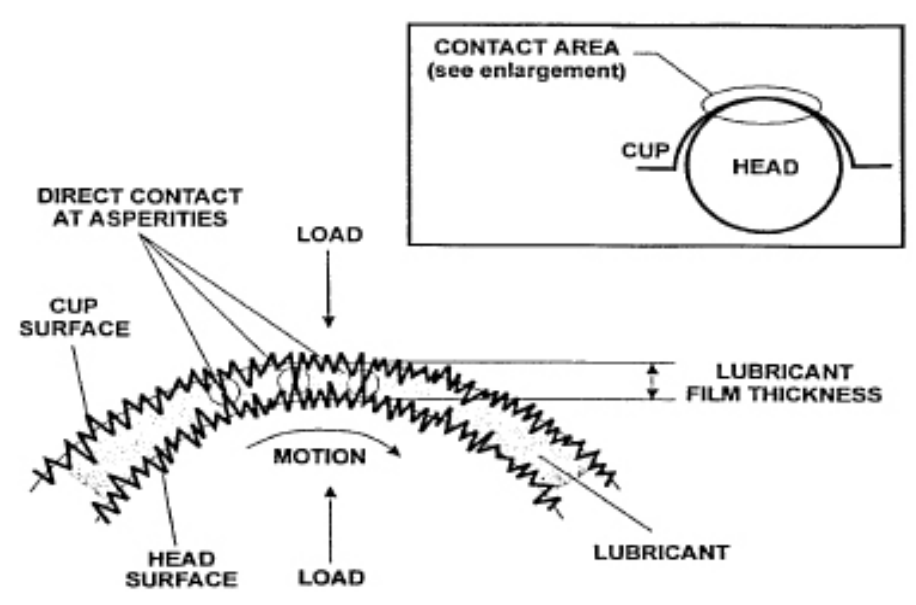

Figure 1.10: Illustration of microscopic rendering of asperity interaction ath the head-cup interface (Chan, Bobyn, Medley, Krygier, \& Tanzer, 1999). responsible for the microscopic

scratches seen on retrieved

implants (Figure1.11) (Willert,

et al., 1996) and is the primary

wear mode present in MoM

orthopaedic implants, generating

a majority of the wear debris

released into the body. This

wear debris is typically needle-

shaped or spherical in shape, depending on the quantity of polishing that has already

occurred on the implant bearing surface. As the implant articulates thousands of times

every day, the two surfaces of the femoral

head and the acetabular cup contact each

other under an applied load. During this

articulation, the surface asperities of each

side of the interface collide (Figure 1.10),

leading to fracture and debris release.

Abrasive wear is particularly high during

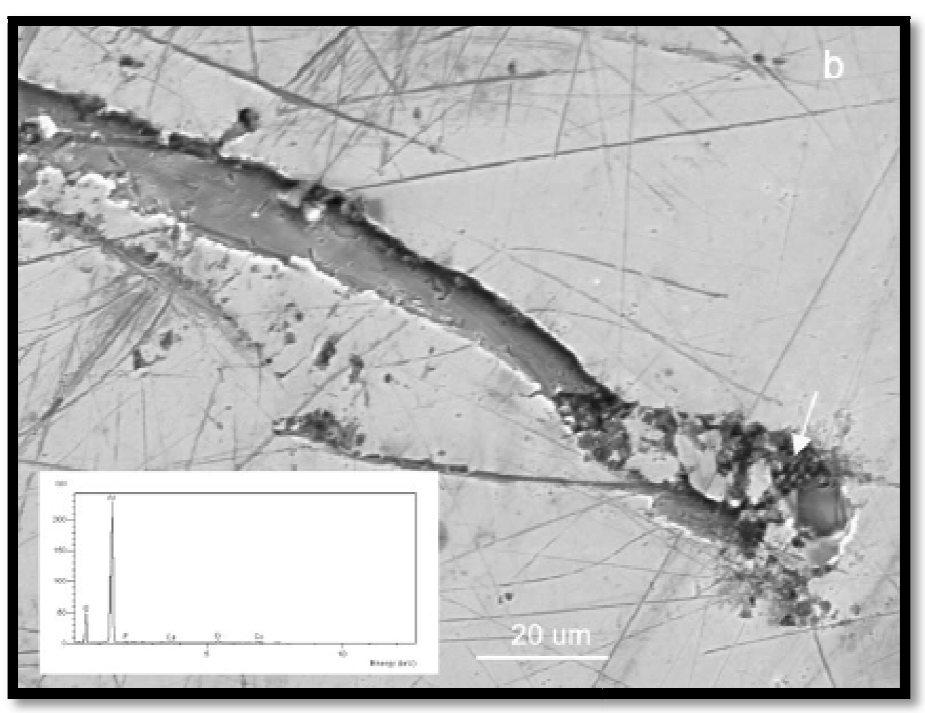

the two year "run-in" period encountered by

all implants, although it reduces

Figure 1.11: A scratch defect on the surface of a CoCr femoral head during articulation. EDS inset shows foreign particle on edge to be Al203. Mag: 1000x. (Milose, Trebse, Kovac, Cor, \& Campbell, 2005). 
significantly once the largest asperities have been removed and the implant surfaces have been polished under load.

Adhesive wear is characterized by the temporary alignment and bonding of asperity peaks at the metal-metal interface. Adhesive wear is generally a larger factor in the production of metal wear debris during intermittent human activity, but can also be a wear generation mechanism during full motion. During adhesive wear, the asperity peaks that are in alignment under load represent the true contact area at the interface and are subjected to significantly greater stress than the nominal contact area of the entire interface (Figure 1.10) (Williams, 2005). At the contact points between asperities, the high pressure leads to physical bonding between the asperity peaks. Because these bonds must be broken for the implant to resume motion, the implant coefficient of friction is increased (Wimmer, Nassutt, Sprecher, Loos, Täger, \& Fischer, 2006). At the asperity bonding site, the lateral shearing of the bonded asperity may take place in a location on the peak other than the bond itself, which can lead to released wear debris if the bond ultimately breaks (Williams, 2005).

The wear particles generated through the abrasive and adhesive wear at the acetabular interface in vivo are typically of nonuniform dimensions and submicron size (Catelas, Campbell, Bobyn, Medley, \& Huk, 2006). The actual diameter of the released wear particles is a subject of some debate, but has been seen to increase with longer implantation times (Catelas, Campbell, Bobyn, Medley, \& Huk, 2006). The majority of recent studies have found metallic wear debris to be in the range of 20 to $100 \mathrm{~nm}$ (Brown, Williams, Tipper, Fisher, \& Ingham, 2006) (Catelas, Medley, Campbell, Huk, \& Bobyn, 2004). In a characterization study, particles were qualified as irregular, but generally 
spherical (Lee, Salvati, Betts, DiCarlo, Doty, \& Bullough, 1992). Regarding particle densities in periprosthetic tissues, Hirakawa et al. (1996) examined tissue samples proximal to failed implants and established an average of $8.5 \times 10^{8}$ to $5.7 \times 10^{11}$ particles per gram (dry weight) of tissue, which was significantly different from the particle concentrations found in the study's control samples at the $95 \%$ confidence level (Hirakawa, Bauer, Stulberg, \& Wilde, 1996). It is important to note that these concentrations were found in failed implants, which often generate significantly more wear than successful implants due to other complicating factors, such as misalignment or non-sphericity (Hirakawa, Bauer, Stulberg, Wilde, \& Secic, 1996). In 1999, Chan et al. found a strong correlation between wear rates and implant surface roughness, microstructure, alignment, and sphericity. In the same study, total volumetric wear rates for the commonly used high carbon cast implants (ASTM F75-92) were determined to be $0.40 \pm 0.11 \mathrm{~mm}^{3} / 3$ million cycles (Chan, Bobyn, Medley, Krygier, \& Tanzer, 1999). By industry standards, these wear rates are considered to be excellent and vastly superior to MoP volumetric wear rates.

\subsection{SYSTEMIC WEAR DEBRIS DisTRIBUTION}

The 10 million wear particles produced annually by an average hip prosthesis (Savarino, et al., Ion Release in Patients with Metal-on-Metal Hip Bearings in Total Joint Replacement, 2002) are dealt with by the body in a variety of ways. In MoP implants, the vast majority of wear debris generated is polyethylene in composition. Polyethylene wear particles are chemically inert and significantly larger than the metal debris released by MoM designs (Hirakawa, Bauer, Stulberg, Wilde, \& Secic, 1996). Because of their large volume, polyethylene wear particles rarely leave the periprosthetic tissue at the implant 
site and gather in significant numbers, ultimately leading to macrophage response and osteolysis. Metal wear debris, primarily cobalt and chromium based particles, can be in the range of $50 \mathrm{~nm}$ (Campbell, Amstutz, Kossovsky, \& Clarke, 1992) and can thus be transported through cell walls, facilitating their exodus from the implant location. This lack of aggregation at the periprosthetic tissue can be both beneficial and harmful to the patient, as it prevents localized immunological response and resulting osteolysis but leads to the potential consequences associated with systemic distribution of wear debris and the consequential corrosion byproducts. These potential consequences are discussed in detail in section 1.9 .

A 1990 study by Campbell et al. found that metal wear particles from implants rapidly spread throughout the body. Metal particulate was found in both the urine and blood serum within days of implantation. Also of note, patients with implant ages in excess of 20 years were found to have 9-fold elevations in serum chromium, 35-fold elevations in urine chromium, and at least 3-fold elevations in serum cobalt when compared with controls. The same study found that short term patients with less than a two year implantation time had 3-fold elevations in serum $\mathrm{Cr}$, 4-fold elevations in urine $\mathrm{Cr}$, and 4-fold elevations in serum Co concentrations when compared to the long-term patients. The authors concluded that slightly higher metal concentrations for the newer implants were likely a product of the run-in phenomenon (Dowson, 2006) discussed previously, which suggests that wear rates are initially high until the implant interfaces smooth themselves and wear rates level off to their lower steady state rates. The statistically significant differences found in implant patient cobalt and chromium concentrations suggest systemic exposure to wear debris corrosion byproduct throughout 
the lymph, circulatory, and excretory systems in the body (Dumbleton \& Manley, 2005). Additional research has shown a sevenfold increase in metal ions in synovial fluid around loose Co-Cr-Mo implants as compared to well-fixed devices, implying that the increase in debris production caused by loose implants leads directly to elevated systemic ion concentrations (Naudie, Roeder, Parvizi, Berry, Eggli, \& Busato, 2009).

Although the volume of wear debris production from MoM implants is low enough to be relatively well tolerated by the body (Merritt \& Brown, 1996), the accumulation over implant times greater than thirty years do concern researchers. In order to prevent gradual accumulation, the body is capable of removing some of the wear debris generated by an implant in the excretory system. Cobalt ions can be removed relatively easily, while chromium debris and ions are stored in tissue and are released more slowly (Liu, Liu, Chang, Yang, \& Rong-Sen, 1998). A study by Merrit et al. showed that almost all of the cobalt ions, when delivered to an animal as a bolus, were recovered in the urine within 48 hours. Chromium is of greater concern, because it is not readily transported to the blood and removed from the body in the urine. Chromium accumulates gradually in tissues and red blood cells, where cellular attempts to eliminate the chromium debris are only partially effective.

\subsection{Corrosion of Cobalt Chromium Alloy Wear Debris}

The generation of wear debris through articulation of orthopaedic implants is a well documented effect and has been thoroughly investigated. This study aims to fill the void in research performed on the actual kinetics of in vivo corrosion or ion dissociation at the submicron particle level once the particulate wear debris has left the implant site and is an independent body. Particles on this scale require a sub-section of corrosion 
studies due to their extremely high surface area to volume ratios and unique interaction effects (Eremenko, 1959). While the basics of corrosion remain identical in terms of the formation of an electrochemical corrosion cell and the development of a current leading to ion dissociation and material loss from the base metal, the rates and ion release may differ drastically enough to induce unanticipated consequences in the body. It is important to note that the process of ions leaving the surface of metal wear debris does not occur through a singular event. Although an excellent umbrella term, corrosion is not always precisely the correct diction when referring to metal ion surface release. The word corrosion implies an electrochemical cell and the occurrence of an oxidation-reduction reaction. Ion dissociation from wear debris can occur as a process driven purely by a free energy differential. While less common than corrosion, ion dissociation is a factor in the production of ions in the body and will be differentiated briefly here.

The driving force for the rise in reactivity and ion dissociation rates of increasingly small particulate matter is a difference in surface energy at the submicron level (Gladstone, 1941). As particles of decreasing diameters are investigated, the role of surface energy gains significance in the reaction cell. A high surface area to volume ratio, as found in submicron particles, leads to a high reactivity because the surface effects dominate the ion dissociation reaction (Eremenko, 1959) (MacRitchie, 1990). Because metal wear debris in orthopaedic implants is on the submicron scale, it will be significantly more reactive than the bulk implant and will lead to a higher than anticipated rate of ion dissociation. This phenomena of increased reactivity helps to explain why metal ion concentrations in MoM implant patients are high, despite minor or nonexistent corrosion of the implant itself. Additionally, wear debris released from the 
implant is often the target of immunological responses by the body and attempts to phagocityze the individual particles lead to depressed localized $\mathrm{pH}$ and further increased corrosion rates (Willert, et al., 1996).

The debris released from MoM implants is varied in nature. Pure chromium, cobalt, and molybdenum particles have been identified (Milose, Trebse, Kovac, Cor, \& Campbell, 2005) in addition to oxidation byproducts such as chromium orthophosphate (Papageorgiou, et al., 2007). These particles leave the surface of the implant through a variety of wear mechanisms and are transported systemically by the circulatory and lymph systems. Although elevated cobalt concentrations may have negative effects, the debris variety that most concerns researchers is chromium, as it is gradually accumulated in the body tissues, rather than released in the urine as cobalt and molybdenum are. Chromium particles that accumulate in the red blood cells and body tissues are known to gradually dissociate into principally hexavalent chromium $\left(\mathrm{Cr}^{+6}\right)$ and trivalent chromium $\left(\mathrm{Cr}^{+}\right.$) (Merritt \& Brown, 1996). This is reason for concern because hexavalent chromium is considered to be among the most toxic of chromium valence states (Occupational Safety \& Health Administration, 2008).

\subsection{CONSEQuences of Wear Debris and Resulting Metallic Ions}

The systemic elevation in metal ion concentrations after a MoM hip replacement is a well researched fact (Campbell, Amstutz, Kossovsky, \& Clarke, 1992) (Baslé, Bertrand, Guyetant, Chappard, \& Lesourd, 1996) (Catelas, Medley, Campbell, Huk, \& Bobyn, 2004); however, the body's response to these ions remains unclear. Metal ions introduce an additional risk beyond polyethylene wear debris; because they may be released at different sites in varying amounts, due to the systemic nature of the metal 
wear products of implant articulation. As a consequence of this completely systemic ion distribution, many biological functions are affected, inducing both local and broad effects that can influence the implant success. Adverse tissue reactions, cytotoxicity, carcinogenesis (Michel, Noite, Reich, \& Loer, 1991), and metal sensitivity are the principle undesired effects of metal wear debris and its corrosion products in the body (Savarino, et al., 2000).

The effects of elevated ion concentrations can be classified as either local or systemic. Local effects are typically characterized by the metal wear debris covering the tissue surrounding the implant, which is known as metallosis. Systemic effects are caused by dissolved ions or transported debris travelling to other regions of the body. In 1995, J.D. Bumgardnerl and L.C. Lucas performed an applicable study on cellular response to metal ions from surgical implants. The results of their testing showed that while the presence of metal ions did decrease cellular proliferation, they did not affect cell morphology or viability. This study is applicable to cellular response to metal ions throughout the body. A contradictory study, performed in 1997, simulated systemic distribution of metal ions by incubating human macrophage cells with high concentrations of $\mathrm{Cr}^{+3}$ and $\mathrm{Cr}^{+2}$ ions. Cell damage was determined to be linearly related to time and necrosis was predominant after only 48 hours, although the concentrations of ions used were far in excess of those seen in MoM patients (Lee, Brennan, Jacobs, Urban, Ragasa, \& Glant, 1997).

In terms of localized effects, a 2007 study by Papageorgiou et al. tested whether patients exposed to worn cobalt chrome hip replacements show an increase of chromosome aberrations in the bone marrow adjacent to the implant and an increase of 
chromosome translocations and aneuploidy, or incomplete chromosome reproduction, in the peripheral blood. After 15 days of exposing human fibroblasts of varying ages to significant quantities of CoCr alloy particles, DNA damage and chromosome translocations could be identified. The cellular reaction to this DNA damage included loss of viability, induction of senescence, and a lesser rate of mitosis (Papageorgiou, et al., 2007).

With respect to the potential for an elevated cancer risk for implant patients, a 1996 study found that the long-term biological effects of a MoM implant could include chemical carcinogenesis and that the total risk of cancer was found to be 1.23 -fold higher in patients who had MoM THR than that experienced by patients with MoP implants (Savarino, et al., 2002). Additionally, evidence of a statistically significant increase in leukemia as well as both prostate and kidney cancers have been shown in MoM patients (Granchi, et al., 1998). In spite of these studies, a study of 31,651 Finnish hip replacement recipients showed no statistically significant indication of cancer risk for MoM patients (Rae, 1981). Although $\mathrm{Cr}^{+6}$ and $\mathrm{Cr}^{+3}$ are classified as class 1 and 2 carcinogens by the International Agency for Research on Cancer and strong evidence exists to show that cell necrosis and DNA damage can occur through exposure to elevated concentrations of metal ions (Jakobsen, et al., 2007), there is yet to be conclusive evidence that the specific quantity of metal ions released by MoM implants causes carcinogenesis or other adverse reactions. There is also little research that can accurately portray how many ions are truly released by the wear debris ejected from MoM implants.

\subsection{RESEARCH STATEMENT}


The intent of this research is to determine the dependence of ion dissociation rate and corrosion kinetics on the surface area to volume ratio of wear debris ejected from bulk MoM hip implants. The completion of this goal will require the development and use of an experimental methodology that will be verified through the corrosion of a variety of particle sizes, the results of which will be extrapolated to actual implant wear debris. The specific objectives of this research can be summarized in the following four goals:

1) Design, build, and test an apparatus for the quantification of an ion release rate from orthopaedic wear in simulated in vivo conditions.

2) Create an experimental methodology to accurately and repeatably evaluate the ion release rate of a specimen that represents $\mathrm{CoCr}$ wear debris.

3) Analyze data in order to establish an actual ion release rate from representative cobalt chromium alloy particles.

4) Relate the specimen ion release rate to particle surface area to volume ratio. 


\section{MATERIALS AND METHODS}

\subsection{TeSt Plan Overview}

The validation of the experimental method designed for the first goal of this research project was performed by immersing ASTM F75 cobalt chromium alloy, hereafter $\mathrm{CoCr}$, spheres of known geometric dimensions and tolerances into an electrolyte and measuring ion release using spectrophotometry. The CoCr samples were divided into two separate groups by diameter: $400 \mu \mathrm{m}$ and $40 \mu \mathrm{m}$. Samples of each diameter group were weighed to specific mass loadings and added to an electrolyte solution in a closed sample vial for testing. Test samples were agitated for predetermined time durations, after which the electrolyte ion concentration was analyzed and the particles were investigated for corrosion effects using visual, compositional, and mass loss analysis.

\subsection{MATERIALS}

The CoCr spheres used as representative wear particles were provided by the UCLA Joint Replacement Institute. The two diameters of spheres utilized for the experimentation were $40 \mu \mathrm{m}$ (standard deviation: $12.7 \mu \mathrm{m}$ ) and $400 \mu \mathrm{m}$ (standard deviation: $9.8 \mu \mathrm{m}$ ) (Figure 2.1). This difference in sphere diameter was considered to be relevant enough to produce significantly different ion dissociation rates and validate the test methodology through comparison. The simulated wear debris was reported to be of ASTM F75 specifications (Table 2.1) (Krokslätts, 2007), which was confirmed using energy dispersive spectroscopy analysis. 


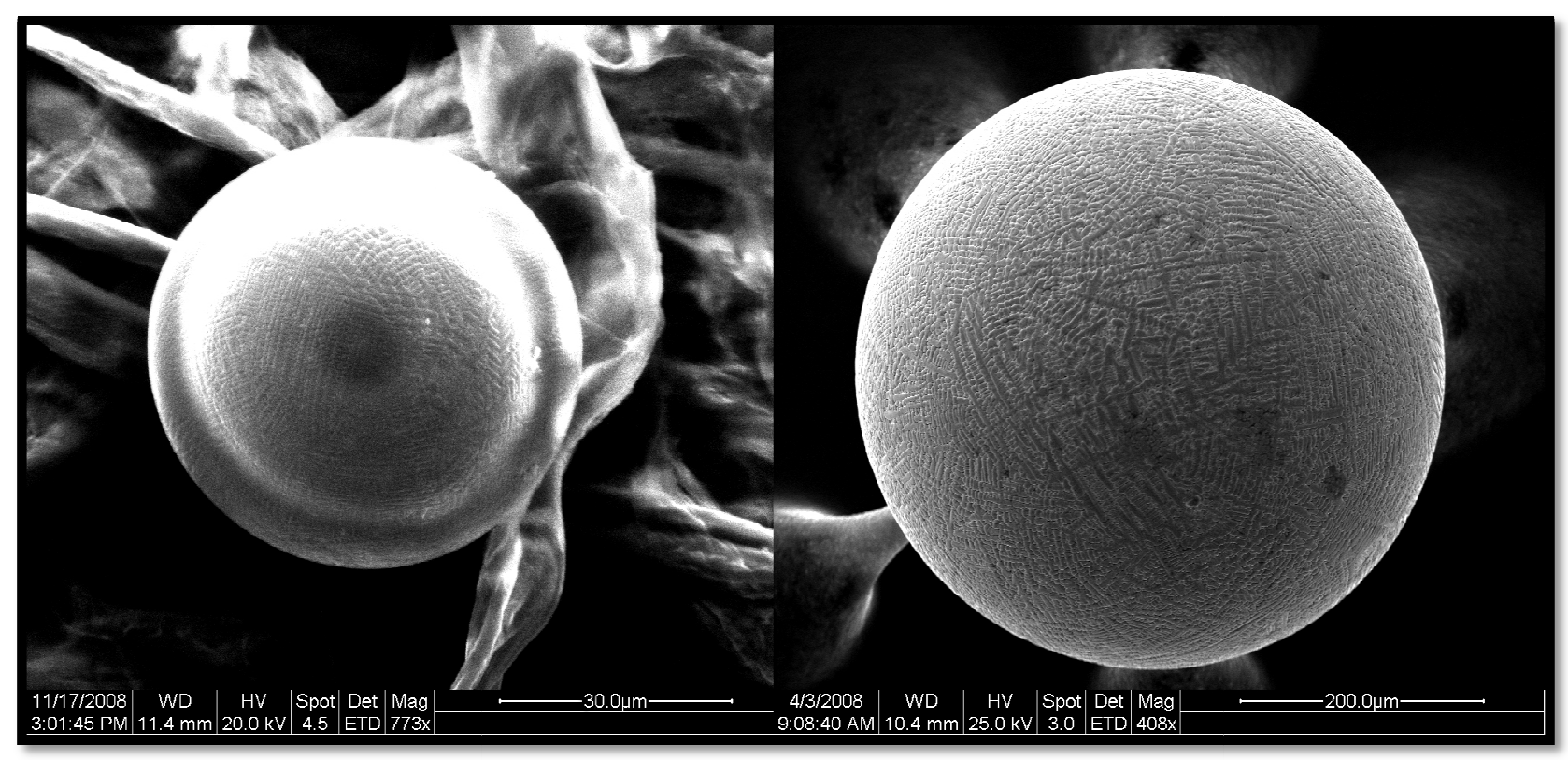

Figure 2.12: $40 \mu \mathrm{m}$ (left) and $400 \mu \mathrm{m}$ (right) diameter CoCr spheres used for testing. Note the cast dendritic structure, geometrically spherical shape, and similarity between samples.

\begin{tabular}{|l|l|l|l|l|}
\cline { 2 - 5 } \multicolumn{1}{c|}{} & \multicolumn{3}{l|}{$\begin{array}{l}\text { Table 2.1: Comparison between as } \\
\text { received samples and ASTM F75 } \\
\text { standard for CoCr. }\end{array}$} \\
\cline { 2 - 5 } \multicolumn{1}{c|}{ Element (Wt. \%) } \\
\hline Sample & Co & Cr & Mo & C \\
\hline ASTM F75 Standard & 66.15 & 27.5 & 6 & 0.35 \\
\hline As received 400um sphere & 64.23 & 27.79 & 4.97 & 1.43 \\
\hline
\end{tabular}

While CoCr alloy implants are typically cast, annealed, and polished according to proprietary treatment schedules, the spheres used to simulate wear debris in this experiment were prepared using a spray solidification method, in which molten $\mathrm{CoCr}$ is sprayed into air or other cooling medium and rapid solidification occurs. The spheres were then briefly solution annealed and sorted according to mesh size.

The electrolytic solutions used for debris immersion were all based on Hank's Balanced Salt Solution (HBSS). Bulk samples of the HBSS were blended with 0.1 molar 
hydrochloric acid to achieve a variety of $\mathrm{pH}$ levels, including $1,3,3.4$, 4, and 7. Blending was performed by adding $1 \mathrm{~mL}$ increments of $\mathrm{HCl}$ to 1 L bulk samples of HBSS (Figure 2.2). The bulk pH was monitored using an Oakton PCD650 pH meter.

Preparing bulk samples of electrolyte prior to immersing the simulated wear debris allowed for negligible variation between samples.

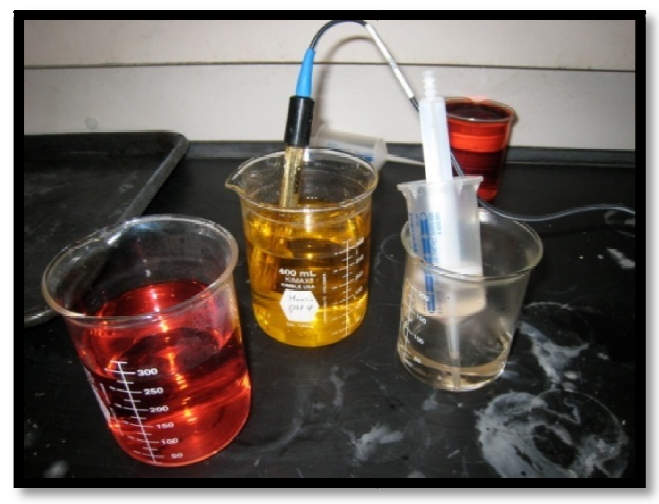

Figure 2.2: Blending the red colored HBSS with $0.1 \mathrm{M}$ $\mathrm{HCl}$ resulted in a yellow colored $\mathrm{pH} 3$ solution.

The $33 \mathrm{~mL}$ HDPE and $22 \mathrm{~mL}$ glass sample vials used for sample containment were purchased from Fisher-Scientific and were commercially sterilized and sealed prior to use, preventing the need for in-house cleaning and potential contamination.

\subsection{SAMPLE PREPERATION}

Initially, the $\mathrm{CoCr}$ sphere samples of different diameters were weighed into 100 mg subgroups, although later tests utilized a variety of mass loadings. After separation, the samples were placed in HDPE or glass vials prior to the addition of the selected electrolytic medium. This weighing and particle transfer was performed as quickly as possible in order to prevent the unnecessary growth of oxides unrelated to the electrolytic medium. A small quantity of oxide formation is considered to be acceptable and does not affect the gradual oxide formation in solution (Virtanen, Kurz-Hochstrasser, \& Hodgson, 2001). The vials were filled with electrolyte in such a way that no air was left remaining

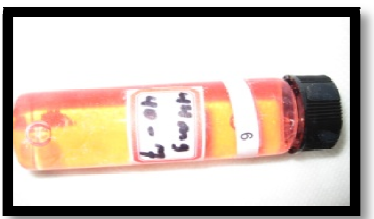

in the headspace and each vial contained exclusively $\mathrm{CoCr}$ spheres and electrolyte (Figure 2.3) . Each vial was shaken to 
separate particles, labeled with the required

information concerning its contents, and placed at

random in the incubating shaker sample holder

(Figure 2.4). The tests were initiated promptly after

loading of the sample holder in order to prevent

aggregation at the vial bottom.

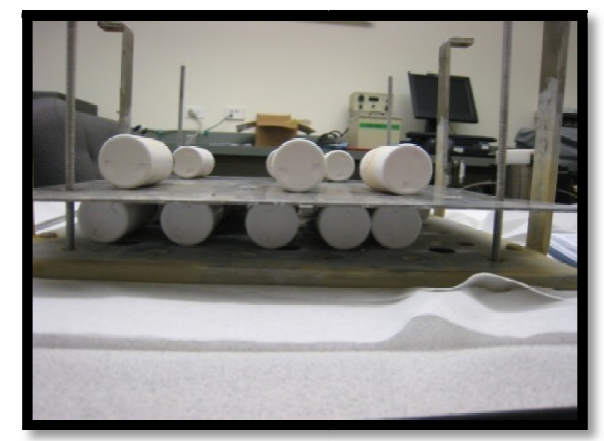

Figure 2.4: HDPE sample vials placed at random in the sample holder prior to compression fastening. The four vertical threaded rods allowed for the use of nuts to compress the samples, effectively holding them in place.

\subsection{Test Methodology}

The first goal of this research study was to design, build, and test an apparatus for the quantification of an ion release rate from orthopaedic wear in simulated in vivo conditions. Therefore, a testing apparatus was constructed to incubate and agitate numerous samples simultaneously at average human body temperature, $37^{\circ} \mathrm{C}$. The incubating apparatus was a New Brunswick G76D (Figure 2.5) incubating orbital shaker in conjunction with a custom fabricated sample holder (Figure 2.6). The sample holder

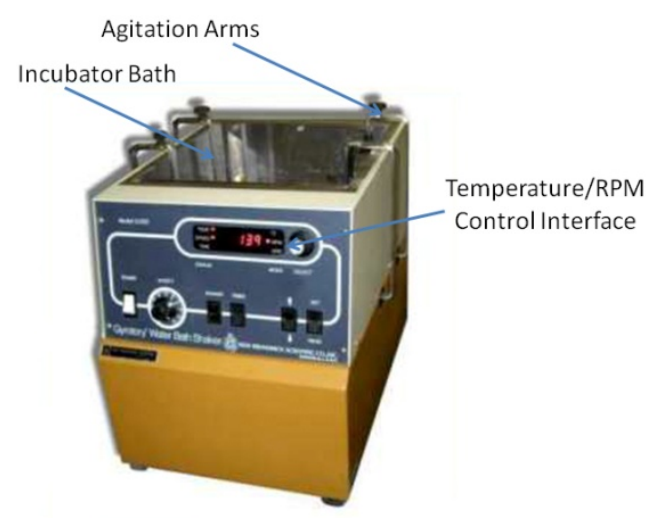

Figure 2.5: New Brunswick G76D incubating shaker. The samples were loaded into the sample holder and attached to the four agitation arms for testing (Image courtesy of New Brunswick). was loaded at random with samples prepared

according to section 2.3. The sample holder, once

loaded, was secured into place via attachment to each of the four agitation arms of the shaker, which was previously filled with $6 \mathrm{~L}$ of $37^{\circ} \mathrm{C}$ water and 8 oz of ethylene glycol as a corrosion inhibitor. The tank was checked for full immersion of the samples (Figure 2.7) prior to initiating the agitation cycle. 
The samples were agitated in the orbital shaker at rates ranging from 100 and $150 \mathrm{rpm}$. Test durations varied from 1 to 42 days and were performed at $37^{\circ} \mathrm{C} \quad 1^{\circ} \mathrm{C}$. A wide variety of $\mathrm{pH}$ levels, including $1,3,3.4,4$, and 7 , were used in the various tests performed. At the end of each test, the simulated $\mathrm{CoCr}$ wear particles were filtered from solution and examined for

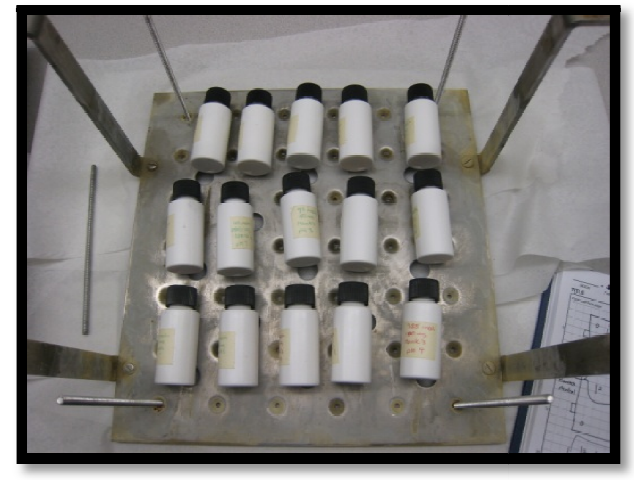

Figure 2.6: Base level of fabricated sample holder. Samples were laid horizontally and secured through compression of aluminum plates above. Flow holes were drilled to allow for adequate distribution of the incubating bath water.

corrosion and wear effects.

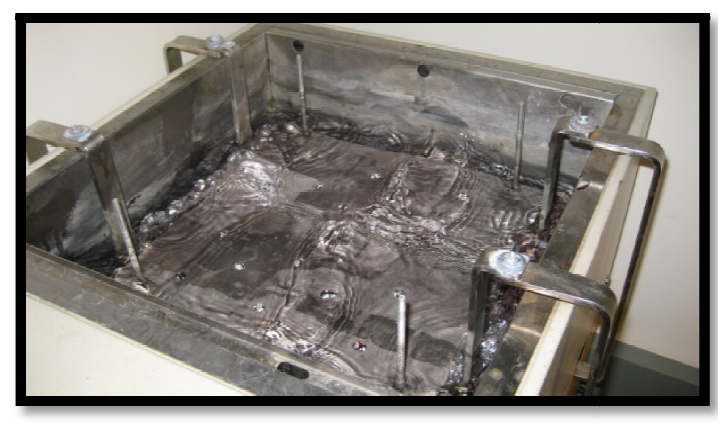

Figure 2.7: Fully submerged sample holder during agitation.
The filtration process selected for consistent and complete particle separation was vacuum filtration using a Millipore filtration system (Figure 2.8), a Hitachi vacuum pump, and $0.2 \mu \mathrm{m}$ filter papers. The filtration system

was prepared by clamping the receiving chamber to the sintered base and sealing the stopper into the Erlenmeyer flask, which was connected to the vacuum pump via reinforced polyvinyl tubing. Each sample vial exterior was individually rinsed with deionized water to prevent external contamination during pouring. Individually, the entire contents of each test vial were poured into the filtration system receiving

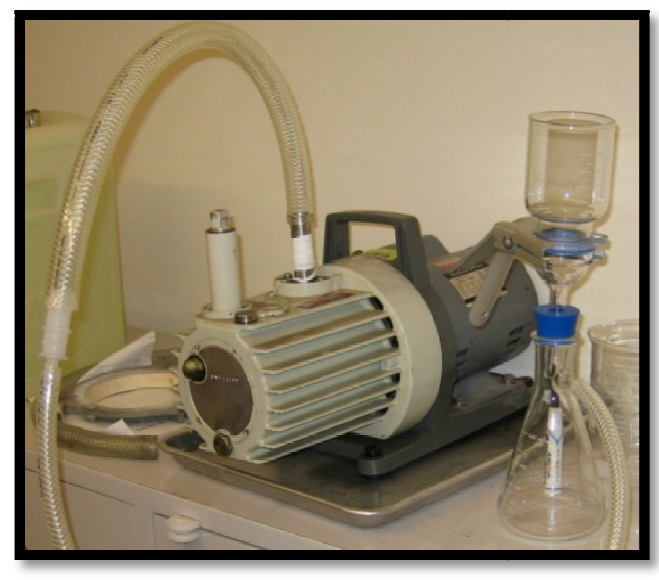

Figure 2.8: Vacuum filtration system used to separate debris from electrolyte post test. The Millipore filtration system is on the right. 
chamber. The empty vials were resealed and set aside for disposal while the vacuum pump was switched into the on position, drawing a vacuum in the Erlenmeyer flask below the filter paper at the sintered base of the receiving chamber (Figure 2.9). Within two seconds, the pressure differential led to the electrolyte being drawn through the filter paper in its entirety, leaving the CoCr spheres on the filter surface. Figure 2.10 shows both a high and low magnification image of the spheres after filtration, illustrating the thorough nature of the separation method. The vacuum pump was left in the on position

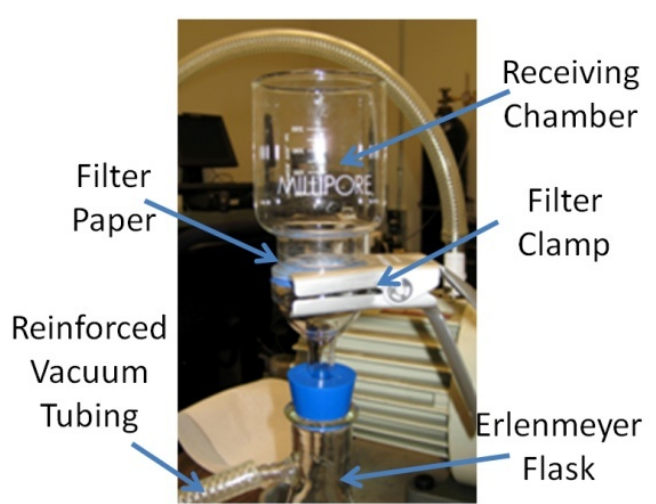

Figure 2.9: The millipore system used for particle filtration. The electrolyte was drawn through the filter paper into the flask by a pressure differential. for a total of seven seconds for each sample in

order to ensure complete electrolyte filtration.

The electrolyte sample within the Erlenmeyer

flask was then emptied into a labeled and commercially sterilized high density

polyethylene (HDPE) medical-grade sample vial, which was then sealed and stored for

prompt ion concentration evaluation. The debris-carrying filter paper was removed from the vacuum filter and isolated in a sealed culture dish for later investigation. The receiving chamber was thoroughly rinsed with deionized water prior to clamping back into position. Once emptied, the Erlenmeyer flask was again secured to the Millipore system and $50 \mathrm{~mL}$ of deionized water was drawn through the system without filter paper in place. This step was performed between every sample in order to clean the filter assembly and prevent cross contamination between samples. Six Erlenmeyer flasks were used in alternating order for the sample extractions. Between each individual vacuum extraction, the Erlenmeyer flasks were thoroughly cleaned with soap and water, rinsed 


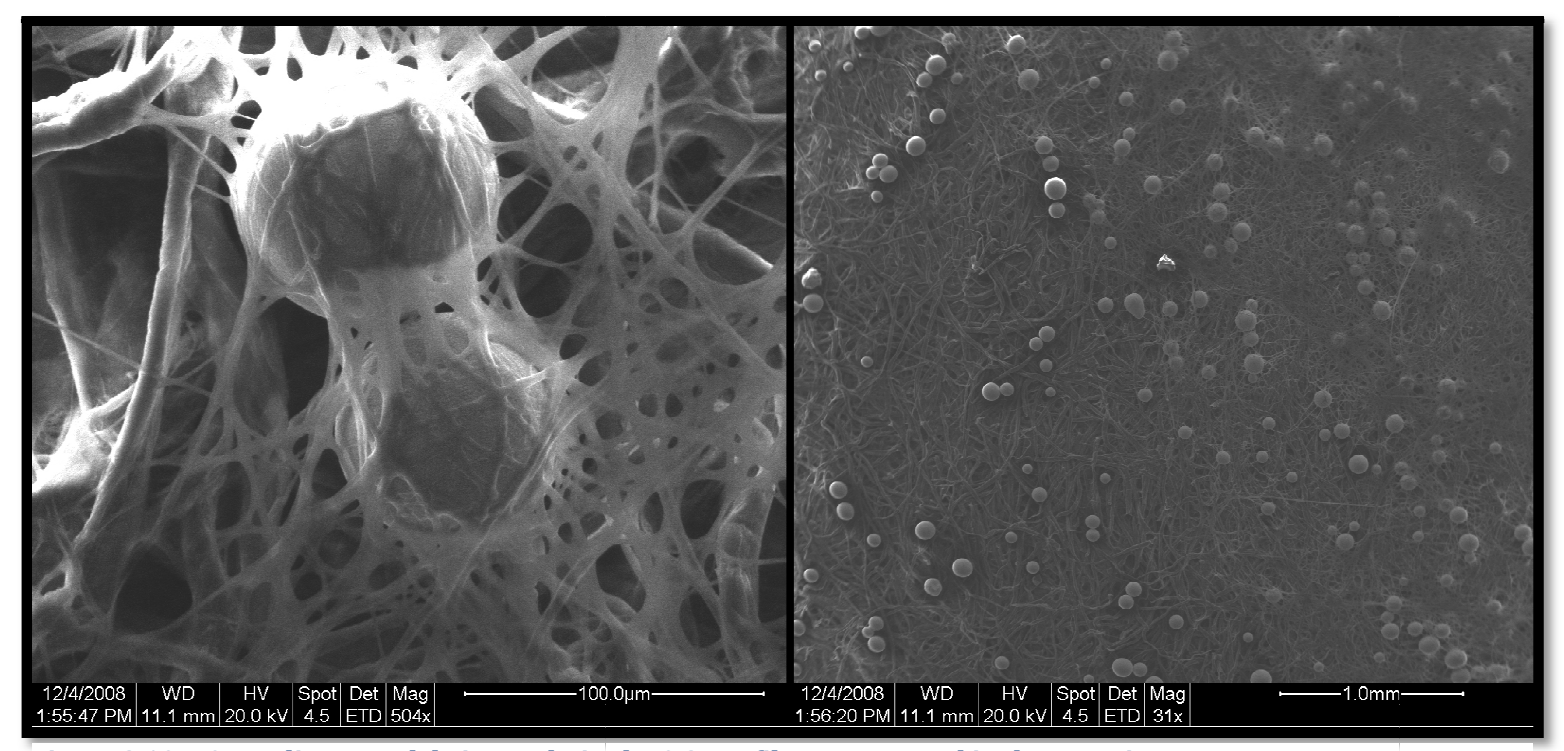

Figure 2.10: $40 \mu \mathrm{m}$ diameter debris caught in the $0.2 \mathrm{um}$ filter paper used in the experiments.

with deionized water, and finally rinsed with ethanol. After cleaning, the flasks were dried on a hot plate prior to each use.

The electrolyte from each sample vial was promptly analyzed for ion content using either a flame atomic absorption spectrophotometer (AAS) or an inductively coupled plasma atomic emission spectrometer (ICP). Both systems require the use of calibration solutions to evaluate concentrations based on light absorbance spectra. Upon instrument calibration, electrolyte samples are drawn in a random run order into the ICP or AAS in $10 \mathrm{~mL}$ increments directly from the sample vial. The AAS and ICP perform analyses on each individual sample, providing concentration results at the completion of each sample run.

Additionally, the wear debris stored on the filter papers was selectively mounted on carbon tape and placed on 3/8" post scanning electron microscope (SEM) mounts. SEM images of samples were used to visually compare surfaces, energy dispersive spectroscopy (EDS) was used to analyze compositional surface morphology, and a focused ion beam (FIB) was used to cross section and analyze sample spheres for oxide 
development. The SEM, EDS, and FIB were used at voltages ranging from 15 to $20 \mathrm{kV}$ and spot sizes ranging from 3 to 4.5 . These parameters were varied during use in order to yield images of optimal quality. 


\section{RESULTS AND DISCUSSION}

\subsection{OVERVIEW OF OBJECTIVES}

The intent of this research is to determine the dependence of ion dissociation rate and corrosion kinetics on the surface area to volume ratio of wear debris ejected from bulk MoM hip implants. The completion of this goal will require the development and use of an experimental methodology that will be verified through the corrosion of a variety of particle sizes, the results of which will be extrapolated to actual implant wear debris. The specific objectives of this research can be summarized in the following four goals:

1) Design, build, and test an apparatus for the quantification of an ion release rate from orthopaedic wear in simulated in vivo conditions.

2) Create an experimental methodology to accurately and repeatably evaluate the ion release rate of a specimen that represents $\mathrm{CoCr}$ wear debris.

3) Analyze data in order to establish an actual ion release rate from representative cobalt chromium alloy particles.

4) Relate the specimen ion release rate to particle surface area to volume ratio.

\subsection{TEST APPARATUS}

The mandatory initial step for the complete project was the design and construction of an experimental apparatus for use in the intended experiments. The testing apparatus was the core of each experiment and its effective operation was critical to the production of repeatable results.

The functional requirements for the test apparatus were listed as follows: 
- Agitate samples between 50 and $200 \mathrm{rpm}$.

- Utilize an orbital radius of no more than 3" and no less than 0.5 ".

- Maintain constant sample temperature of $37^{\circ} \mathrm{C} \pm 2^{\circ} \mathrm{C}$.

- Capable of testing a minimum of 40 individual samples.

- Capable of continuous testing for a minimum of 42 days.

- Complete isolation of sample test medium from heating agent.

Several designs were considered for the testing configuration, but ultimately a modified commercially available system utilizing a heated water bath and employing an orbital agitation system was deemed the best alternative. A New Brunswick Model G76D Orbital Water Bath Shaker was chosen as the foundation for the custom test apparatus

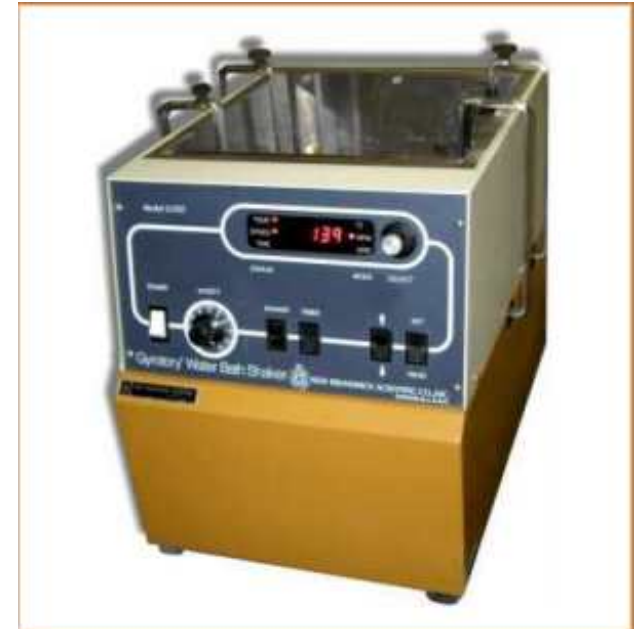

Figure 3.1: The New Brunswick G76D Gyratory Shaker Bath, used to repeatably agitate samples at body temperature. Note the 4 arms extending into the bath cavity, to which a sample holder was attached.
(Figure 3.1). The G76D utilizes a feedback loop between a quartz temperature sensor and a heating element to maintain a constant and uniform bath temperature within $1^{0} \mathrm{C}$. Figure 3.2 illustrates the thermocouple responses for two thermocouples monitoring the bath temperature during the initial heating period to the steady state temperature, which was reached after approximately 80 minutes. Samples were placed in the constant temperature bath and agitated for the test duration

using the equipped 4-arm agitation system. A customized sample holder (Figure 2.6) was manufactured from 1/8" aluminum sheet metal, 3/8" - 24 aluminum threaded rods, and matching aluminum nuts. The sample holder was designed to secure vials through a 
clamping force of approximately $6 \mathrm{~kg}$. The holder was capable of securing in excess of 40 samples with the option for expansion and maintained uniform spacing between sample vials in order to allow for adequate fluid circulation between samples. Additionally, in order to allow for adequate fluid flow vertically between the samples, the sample holder was manufactured with uniformly spaced $1 / 2$ " diameter holes in each of the plates.

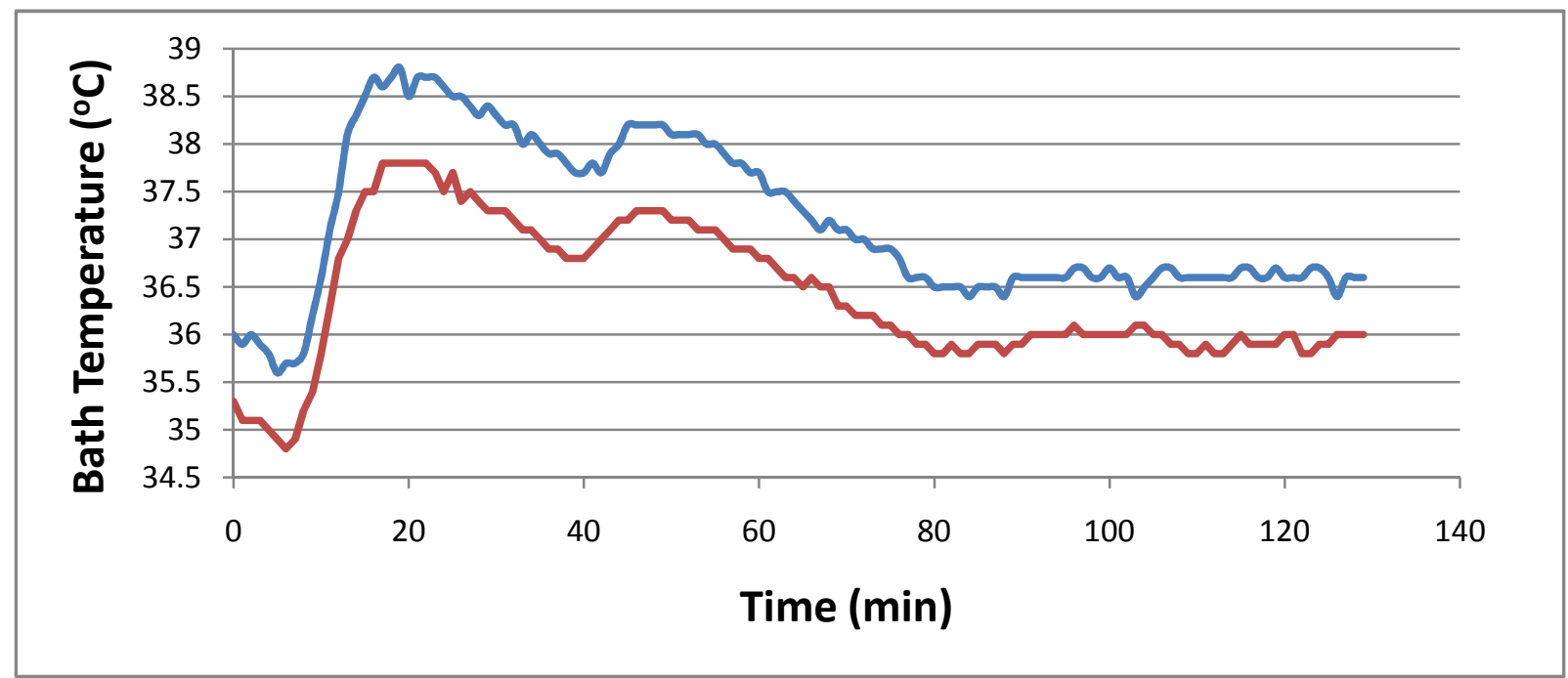

Figure 3.2: In order to validate the uniform heating capacity of the G76D water bath, two thermocouples were placed in the bath. One was placed on the bottom, immediately proximal to the heating element, and the other was placed at the top of the water

The aluminum sample holder was secured to the four steel arms of the G76D agitation system using wing nuts and rubber gaskets in order to prevent the formation of a galvanic cell between the dissimilar metals. The completed test apparatus satisfied the functional requirements specified at the outset of construction and proved to be an effective means of testing $\mathrm{CoCr}$ particles that were representative of wear debris in a simulated body environment.

\subsection{DISCUSSION OF EXPERIMENTAL METHODOLOGY IMPROVEMENTS}

The experimental methodology, described in full in section 2.4 of this document, evolved throughout the project in response to unanticipated changes in the experimental 
environment as well as improvements in the equipment available for use. A research agreement through the Materials Research Laboratory (MRL) at the University of California, Santa Barbara (UCSB) allowed for the use of an improved ion detection system, the sample vials were upgraded to glass for lessened interaction effects, the blends and $\mathrm{pH}$ of the HBSS electrolytes used were modified numerous times, and the test duration was adjusted. All modifications made to the original test plan were performed with the aim of improving the quality of the data in terms of accuracy and repeatability.

The MRL provided the use of an Inductively Coupled Plasma (ICP) for ion detection. An ICP yields results with a $10 \mathrm{ppb}$ detection limit and provides data that is repeatable with a standard deviation of less than $0.001 \mathrm{ppm}$. This vast improvement of detection capability over the Atomic Absorption Spectrometer (AAS) used in initial experiments allowed for better accuracy and precision, as well as assurance that results were not incorrectly reported.

While the original experimental design called for $33 \mathrm{~mL}$ High Density Polyethylene (HDPE) sample containers, this requirement was changed to $22 \mathrm{~mL}$ sterile glass vials with a taller, thinner profile. This design change was made for several reasons, but primarily because the HDPE, although guaranteeing inertness, appeared to have an internal coating that lost adhesion during the extended duration of the test periods. This coating interfered with the data collection process and was deemed undesirable for proper experimental procedure. Additionally, the lesser volume of the glass vials allowed for greater ion concentrations to accumulate over less time with equivalent $\mathrm{CoCr}$ mass loadings. 
As a response to inconclusive experimental data, both the test duration and the $\mathrm{pH}$ of the electrolyte were modified numerous times throughout the course of the project, with the intent of better mimicking the bodily conditions, as well as improving the quantity of ion detection during analysis. Modifying the $\mathrm{pH}$ to accelerate the corrosion of CoCr was supported by several research studies (Virtanen, Kurz-Hochstrasser, \& Hodgson, 2001) (Metikos-Hukovic \& Babic, 2007), but ultimately did not prove necessary. Lengthening the test durations was deemed the ideal way to increase the number and statistical significance of the ions detected, without disrupting the normal ion dissociation kinetics of the sample. Eventually, in accordance with the second research goal, the test methodology discussed in detail in section 2.4 was prepared to accurately and repeatably evaluate the ion release rate of a specimen that represents $\mathrm{CoCr}$ wear debris. This accuracy and repeatability is further discussed in section 3.5.

\subsection{Data Analysis and Ion Release Rate FOR Four METHOdOLOGy VALIDATION STUDIES}

Data from the experiments performed was gathered in several varieties. The majority of the data collected was in the form of ion concentrations within the electrolytic solutions, in which samples were placed for a given duration of testing. Additionally, microscopic visual analysis and a variety of compositional analyses were performed on the samples in order to develop a more complete comprehension of the corrosion and ion dissociation kinetics at the microscopic level. A total of six experiments were performed in progression, with each experiment capitalizing on information gained from its predecessor. 


\subsubsection{EXPERIMENT 1: INITIAL EXPLORATORY STUDY OF METHODOLOGY}

The initial testing was intended to provide basic information about the reliable detection limits and approximate ion yield of the test system. Test data was to be evaluated for opportunities to optimize and control the test procedure. The first test performed consisted of the following test conditions:

Containment: $33 \mathrm{~mL}$ high density polyethylene (HDPE) vials were used for the containment of each individual sample.

Sample sizes: 8 samples were prepared with $400 \mu \mathrm{m}$ diameter $\mathrm{CoCr}$ alloy spheres and 2 samples were prepared as control samples containing no particles.

Loadings: The mass loading levels of spherical particles within each sample were $5 \mathrm{mg}, 10 \mathrm{mg}, 15 \mathrm{mg}$, and $20 \mathrm{mg}(0.15 \mathrm{~g} / \mathrm{L}, 0.3 \mathrm{~g} / \mathrm{L}, 0.45 \mathrm{~g} / \mathrm{L}$, and $0.6 \mathrm{~g} / \mathrm{L})$. Two replications of each loading were used.

Electrolyte: All samples contained Hank's Balanced Salt Solution at a neutral pH of 7 .

Test duration: 21 days

Agitation rate: $46 \mathrm{rpm}$

These conditions were chosen as preliminary testing parameters to provide relevant information regarding the detection limits for the experiment and initial ion dissolution rates. Immediately after conclusion of the test, the sample vials were removed and the media was filtered from the simulated wear debris. Placed in sterile vials for transport, the electrolyte was taken to the AAS and analyzed for ion concentration (Table 3.1). The ion concentration results are discussed below. 
Table 3.1: Experiment 1 results, illustrating a gradual accumulation or memory effect within the AAS, particularly noticeable in $\mathrm{Cr}$ samples. The samples highlighted orange were above $0.07 \mathrm{ppm}$, and the highest detected concentration was highlighted red.

\begin{tabular}{|l|l|l|l|}
\hline $\begin{array}{l}\text { Sample } \\
\text { Description }\end{array}$ & $\begin{array}{l}\text { Sample } \\
\text { Loading } \\
\text { (g/L) }\end{array}$ & $\begin{array}{l}\text { Chromium } \\
\text { lon Conc. } \\
\text { (ppm) }\end{array}$ & $\begin{array}{l}\text { Cobalt lon } \\
\text { Conc. } \\
\text { (ppm) }\end{array}$ \\
\hline Hanks 1 & 0.15 & 0.066 & 0.010 \\
\hline Hanks 2 & 0.3 & 0.065 & 0.011 \\
\hline Hanks 3 & 0.45 & 0.067 & 0.019 \\
\hline Hanks 4 & 0.6 & 0.066 & 0.033 \\
\hline Hanks 5 & 0.15 & 0.069 & 0.021 \\
\hline Hanks 6 & 0.3 & 0.072 & 0.021 \\
\hline Hanks 7 & 0.45 & 0.072 & 0.014 \\
\hline Hanks 8 & 0.6 & 0.076 & 0.037 \\
\hline Control 1, Hanks & - & 0.074 & 0.024 \\
\hline Control 2, Hanks & - & 0.081 & 0.033 \\
\hline
\end{tabular}

There was no statistical difference found between the loaded samples and the control samples, which was not anticipated. While inconclusive, the results from the first experiment did introduce several critical concepts regarding the experimental methodology.

The foremost concern regarding the results can be seen in the high $\mathrm{Cr}$ and $\mathrm{Co}$ concentrations detected in the control samples. This finding indicates four possibilities: the ion levels were below the AAS detection levels, the solutions used to calibrate the AAS were incorrectly prepared, the experimental methodology was out of control in terms of isolating the experiment from environmental contamination, or the AAS uptake system gradually built up ions that were not flushed and falsely increased subsequent measurements, which is illustrated by the color gradient in Table 3.1. Without further analysis, none of these possibilities could be proven statistically, indicating the need for a new array of experiments to isolate the causes for error. 


\subsubsection{EXPERIMENT 2: PROVING DETECTABLE CORROSION}

Due to the lack of statistically significant results found in the initial experiment, there was considerable concern that the experimental methodology was out of control or contained grievous errors, or that the detection limits of the atomic absorption spectrophotometry equipment used were grossly overestimated. Evidence was required to confirm that metal ions could be detected accurately and at levels significantly greater than control samples. The second experiment conducted was directed primarily at proving the detectable nature of corrosion within the sample cell. The conditions used for the second test were:

Containment: $33 \mathrm{~mL}$ high density polyethylene (HDPE) vials were used for the containment of each individual sample.

Sample sizes: 6 samples were prepared with $400 \mu \mathrm{m}$ diameter $\mathrm{CoCr}$ alloy spheres and 1 sample was prepared as a control sample with no spheres.

Loadings: Each sample was prepared with $50 \mathrm{mg}$ of $\mathrm{CoCr}$ alloy, equivalent to a mass density of $1.5 \mathrm{~g} / \mathrm{L}$ in solution.

Electrolyte: All samples contained $30 \% \mathrm{HCl}$ and $70 \% \mathrm{H}_{2} \mathrm{O}$ with a pH of 0.1 . Test duration: 1 day Agitation rate: $0 \mathrm{rpm}$

The second test was performed by placing $50 \mathrm{mg}$ of CoCr spheres into HDPE sample vials and adding $33 \mathrm{~mL}$ of hydrochloric acid. Do to the hazardous nature of the contents and the existing lack of applicability to the human body; the sample was not agitated, but left immobile in a fume hood. The corroded spheres were filtered from the electrolyte after 24 hours, and the electrolyte was then analyzed in the AAS. The cobalt 
ion concentration was statistically different from the control sample analyzed (p-value: 0.000), as shown in Figure 3.3.

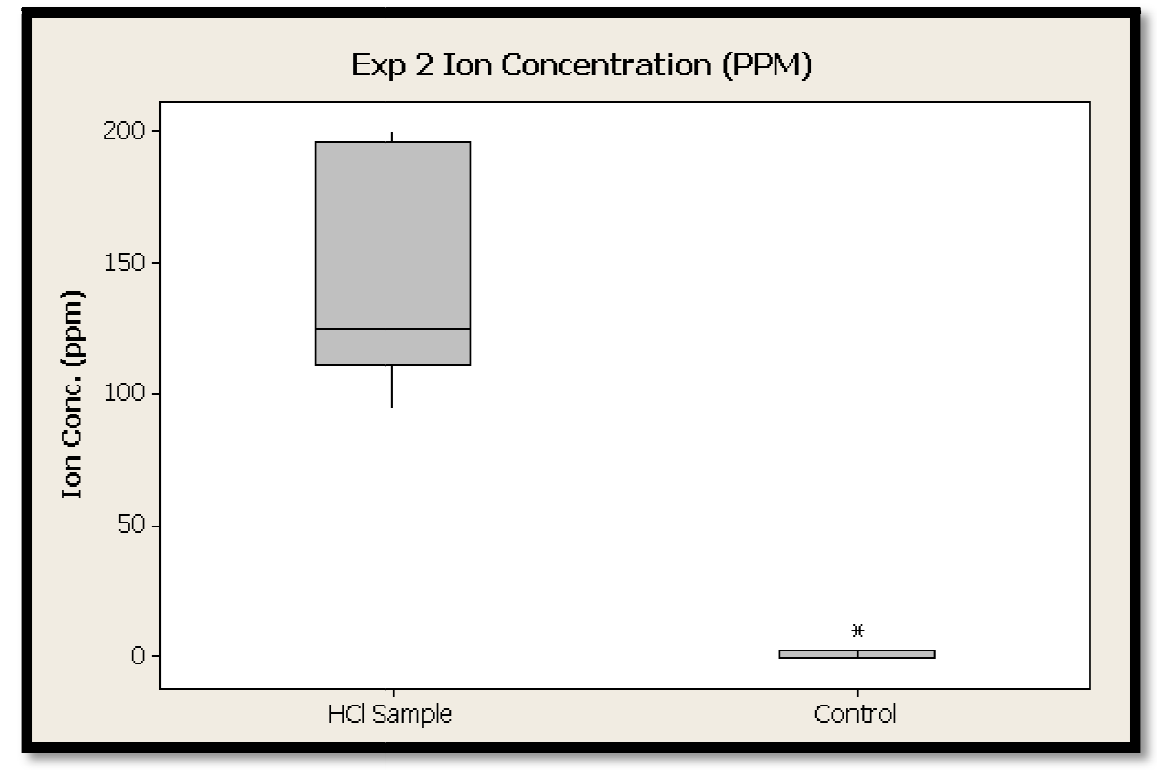

Figure 3.3: The cobalt ion concentrations detected in the $\mathrm{HCl}$ electrolyte after only 1 day were statistically significant.

While the results of the second experiment appeared to confirm the ability of the instrumentation to detect ions in solution, further analysis was required to validate that the spheres were truly corroding. An FEI scanning electron microscope (SEM) was used to investigate the nature of the corrosion that took place at the surface of the representative wear particles. When compared to original, uncorroded $\mathrm{CoCr}$ spheres, the particles from hydrochloric acid immersion (Figure 3.4) exhibited clear preferential corrosion within the dendrite structures, confirming corrosion of the particles, as suggested by the AAS analysis. While it was clear that corrosion had occurred preferentially, it was not immediately apparent what the precise mode of mass loss was or which constituents of the alloy had become most susceptible to corrosion. 


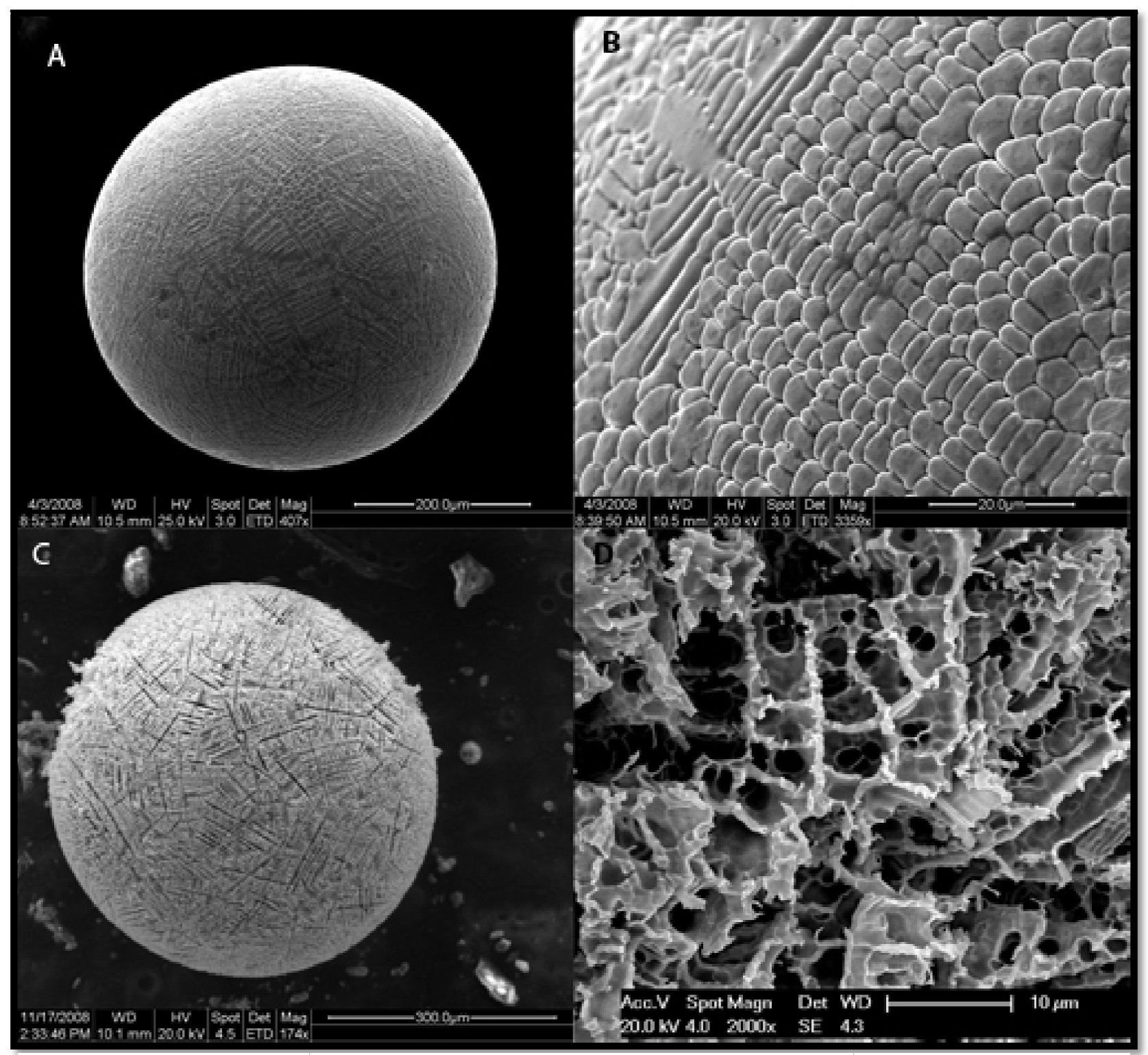

Figure 3.4: Uncorroded CoCr spheres, showing a cast, dendritic structure (A \& B), as compared to the same spheres after 1 day of immersion in $\mathrm{HCl}$ (C \& D). Clear preferential corrosion can be identified well below the surface of the sphere.

After documenting the extensive corrosion and mass loss that occurred during the particle immersion in $\mathrm{HCl}$, energy dispersive spectrophotometer (EDS) was used to develop a further understanding of the preferential corrosion mechanism and kinetics of ion dissociation. The EDS provides compositional data in the form of spectral analysis 
via characteristic radiation ejected from the sample surface during electron bombardment. EDS analysis was performed on both the uncorroded spheres as well as the samples submerged in $\mathrm{HCl}$ (Table 3.2) in order to draw comparative conclusions about the compositional changes during immersion in an electrolyte.

Table 3.2: Composition of corroded and uncorroded $400 \mu \mathrm{m}$ diameter spheres as compared to the ASTM specification for alloy F 75.

\begin{tabular}{|l|c|c|c|c|c|}
\cline { 2 - 7 } \multicolumn{1}{c|}{} & \multicolumn{5}{c|}{ Element (Wt. \%) } \\
\hline \multicolumn{1}{c|}{ Sample } & Co & Cr & Mo & C & 0 \\
\hline ASTM F75 Standard & 66.15 & 27.5 & 6 & 0.35 & - \\
\hline Uncorroded 400um & 64.23 & 27.79 & 4.97 & 1.43 & 1.01 \\
\hline Corroded (HCl) 400um & 35.32 & 33.87 & 13.24 & 15.05 & 1.56 \\
\hline
\end{tabular}

Compositionally, all uncorroded samples compared favorably with ASTM F-75 standard for medical grade cobalt chromium molybdenum alloy (Table 3.2). A notably significant difference occurred between the samples immersed in $\mathrm{HCl}$ and the uncorroded samples. According to the EDS results for the specimens in $\mathrm{HCl}$, cobalt weight percent in the alloy had fallen from approximately $61 \%$ to $35.32 \%$. During the same immersion period, little to no apparent mass loss of chromium or other constituents had taken place. A visual representation of the elemental composition at the sample surface was provided by EDS elemental mapping, which overlays maps of the detected locations of different elements, represented by different colors, onto the original SEM image (Figure 3.5). The overlaid maps in Figure 3.6 illustrate the approximately equal nature of the $\mathrm{Co}$ and $\mathrm{Cr}$ concentrations at the alloy surface, confirming the spectral analysis in Table 3.2 and corroborating the suggestion that $\mathrm{Co}$ is the primary dissociated species. 


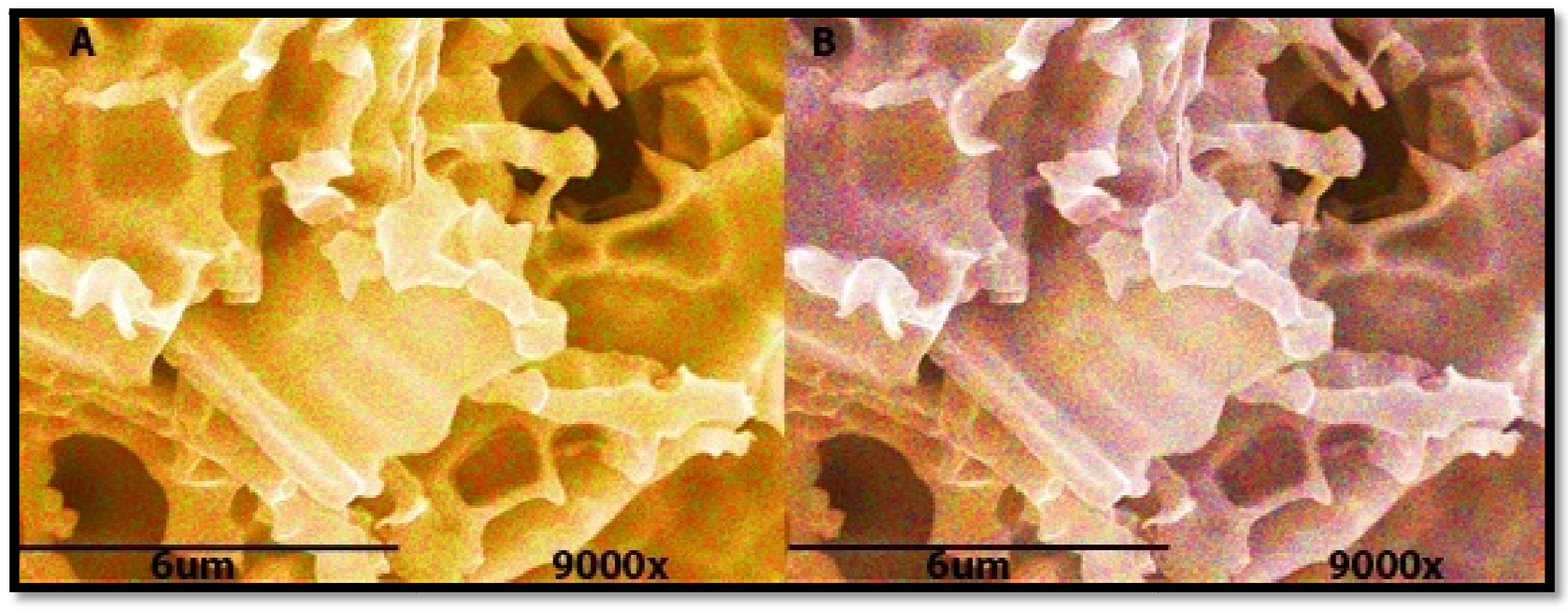

Figure 3.5: EDS compositional mapping of the surface of a sphere corroded in $\mathrm{HCl}$. In the left image (A), red pixels represent a location of predominantly chromium and green pixels highlight cobalt. In this case, chromium and cobalt are approximately equal, confirming the compositional results that $\mathrm{HCl}$ immersion led to a significant drop in cobalt content, implying preferential cobalt corrosion. Additionally, the right image (B) includes blue pixels, which represent oxygen. The relatively high surface oxygen concentrations led to further investigation into oxide formation during corrosion.

The EDS mapping of the corroded surface, particularly when compared to

mapping of uncorroded samples (Figure 3.6) assists in identifying the occurrence of

preferential corrosion of regions high in cobalt content,

as indicated by the ion concentration results.

Unexpectedly, the grain boundaries of the alloy did not

corrode at a higher rate than the grains themselves. On

the contrary, due to the high chromium content at the

grain boundaries seen in Figure 3.6, the individual

grains lost cobalt content from the grain center at a

higher rate than from the grain boundaries. The

microsegregation of higher chromium content along the

grain boundaries, seen in the form of blurred dendrite

edges in Figure 3.6, is likely due to the precipitation of

chromium carbides, specifically $\mathrm{M}_{23} \mathrm{C}_{6}$ carbides, as well

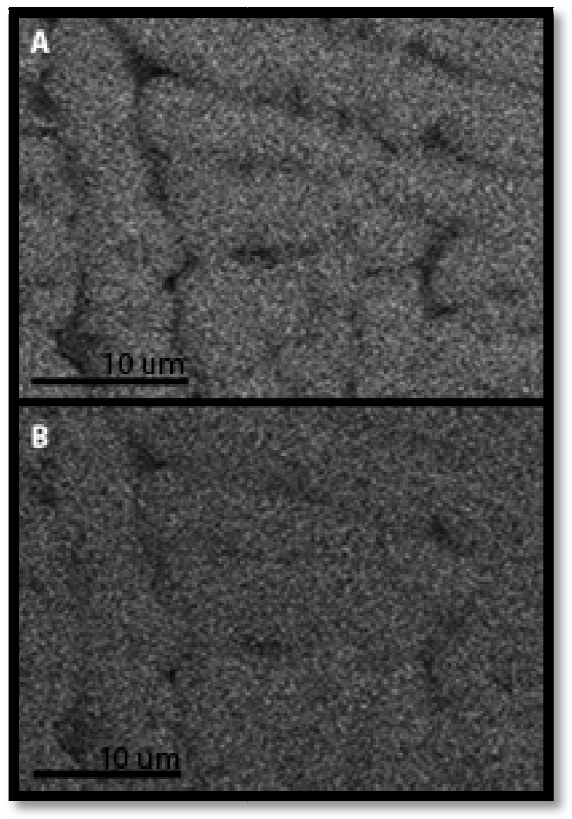

Figure 3.6: (A) EDS elemental mapping of exclusively cobalt on the surface of an uncorroded sample. Note the clarity of grain boundary definition. (B) EDS elemental mapping of chromium on the same image with the same contrast. The definition of the grain boundaries is much less clear, indicating a generally higher $\mathrm{Cr}$ concentration around the boundaries. 
as the formation of a high chromium $\sigma$ intermetallic phase (Brune, Hultquist, \& Leygraf, 1984; 92). Although the alloy is typically solution annealed prior to implantation, the final product cannot achieve $100 \%$ uniformity and the grain boundaries remain the regions with the highest chromium content (Figure 3.7). Although this discovery of increased corrosion from the grain interior over the grain boundary is significant in the development of corrosion science regarding $\mathrm{CoCr}$ alloys and is not discussed in the literature reviewed for this thesis, it is a finding that may be insignificant in true implant wear debris; as such debris is not composed of organized dendritic structures.

Ultimately, because of the lowered chromium percentage within the grain, the

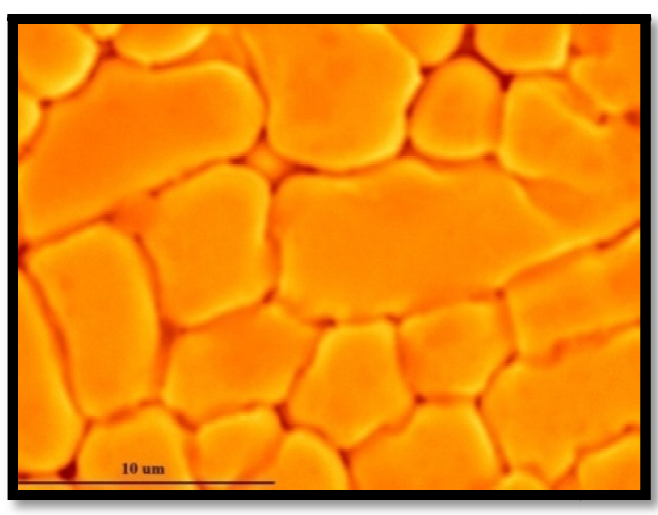

Figure 3.7: EDS rendering of a composite map, illustrating regions with the highest $\mathrm{Cr}$ content as those lightest in color. grain interior becomes the anodic site within the corrosion cell and loses cobalt mass quickly, leaving the heavily corroded sphere seen in Figure 3.4. Although this comprehension of the ion dissociation kinetics of $\mathrm{CoCr}$ alloy in $\mathrm{HCl}$ is not necessarily applicable to implant wear debris in vivo, it does provide an exaggerated perspective

on how ion concentrations might appear for studies applicable to the human body, as well as help to validate the accuracy of future measurements, should they yield similar trends.

\subsubsection{EXPERIMENT 3: LOW STATISTICAL POWER, BROAD RANGE OF CONDITIONS} With the knowledge that the AAS was theoretically capable of detecting Co and $\mathrm{Cr}$ ion levels at a resolution of $0.1 \mathrm{ppm}$, a third experiment was prepared. The third procedure deviated from the first in terms of statistical power. A modified $3^{3}$ factorial design was used with fewer replications than previous experiments. The intention of this 
low power design was to analyze a broad spectrum of loading conditions to identify the corrosion environment that would produce detectable ion concentrations, while still simulating in vivo conditions and requiring a minimal number of samples. The conditions used in this low statistical power study were as follows:

Containment: $33 \mathrm{~mL}$ high density polyethylene (HDPE) vials were used for the containment of each individual sample.

Sample sizes: 9 samples were prepared with $400 \mu \mathrm{m}$ diameter spherical $\mathrm{CoCr}$ alloy particles and 9 samples were prepared with $40 \mu \mathrm{m}$ diameter spheres. Control samples with no particles were prepared with the deionized water used to rinse vials and each of the three pH levels of Hank's Balanced Salt Solution (HBSS) used.

Loadings: The three mass loadings used were $20 \mathrm{mg}, 50 \mathrm{mg}$, or $100 \mathrm{mg}$ (equivalent to $0.6 \mathrm{~g} / \mathrm{L}, 1.5 \mathrm{~g} / \mathrm{L}$, or $3 \mathrm{~g} / \mathrm{L}$ particle densities in solution) of the designated diameter of $\mathrm{CoCr}$ alloy sphere.

Electrolyte: The three levels of $\mathrm{pH}$ used were 3, 4, and 7.

Test duration: 35 days

Agitation rate: $100 \mathrm{rpm}$

The critical differences between the third and first experiments were the duration of the test, the agitation rate, and the variety of factors and levels. All of these modifications were made with the intention of finding detectable ion concentrations and determining the optimal conditions in which that corrosion would take place. Due to the low power of the procedure, the results of the experiment (Table 3.3) were not 
statistically significant, although they did provide some basic insight into the ideal corrosion environment to pursue for future testing.

Table 3.3: Tabled results from the third experiment. Results coinciding with the hypothesis of higher dissociation rates for smaller diameter debris are highlighted in red. Absorptivity measurements were used because only comparisons between samples were made and concentration calculations were unnecessary and introduce error.

\begin{tabular}{|c|c|c|c|c|c|c|}
\hline & & & Chromiun & bsorptivity & Cobalt $A b$ & rptivity \\
\hline $\begin{array}{l}\text { Sample } \\
\text { Number }\end{array}$ & Electrolyte, $\mathrm{pH}$ & $\begin{array}{l}\text { Sample } \\
\text { loading } \\
\text { (g/L) }\end{array}$ & $\begin{array}{l}400 \mu \mathrm{m} \\
\text { Diameter }\end{array}$ & $\begin{array}{l}40 \mu \mathrm{m} \\
\text { Diameter }\end{array}$ & $\begin{array}{l}400 \mu \mathrm{m} \\
\text { Diameter }\end{array}$ & $\begin{array}{l}40 \mu \mathrm{m} \\
\text { Diameter }\end{array}$ \\
\hline 1 & Hanks, $\mathrm{pH} 7$ & 0.6 & 0.001 & 0.001 & 0.0017 & 0.0019 \\
\hline 2 & Hanks, pH 7 & 1.5 & 0.0009 & 0.001 & 0.0013 & 0.0017 \\
\hline 3 & Hanks, $\mathrm{pH} 7$ & 3 & 0.0009 & 0.0008 & 0.0024 & 0.0028 \\
\hline 4 & Hanks, pH 4 & 0.6 & 0.0019 & 0.0014 & 0.0021 & 0.0027 \\
\hline 5 & Hanks, pH 4 & 1.5 & 0.0019 & 0.0014 & 0.0021 & 0.0028 \\
\hline 6 & Hanks, pH 4 & 3 & 0.0021 & 0.0015 & 0.0057 & 0.0038 \\
\hline 7 & Hanks, $\mathrm{pH} 3$ & 0.6 & 0.0015 & 0.0019 & 0.0017 & 0.0043 \\
\hline 8 & Hanks, $\mathrm{pH} 3$ & 1.5 & 0.0015 & 0.0018 & 0.0033 & 0.0038 \\
\hline 9 & Hanks, $\mathrm{pH} 3$ & 3 & 0.0018 & 0.0019 & 0.004 & 0.0086 \\
\hline 10 & Control, DI Water & 0 & 0.0003 & & -0.0001 & \\
\hline 11 & Control, Hanks, pH 7 & 0 & 0.0007 & & 0.0009 & \\
\hline 12 & Control, Hanks, pH 4 & 0 & 0.0007 & & 0.0014 & \\
\hline 13 & Control, Hanks, pH 3 & 0 & 0.0012 & & 0.0014 & \\
\hline
\end{tabular}

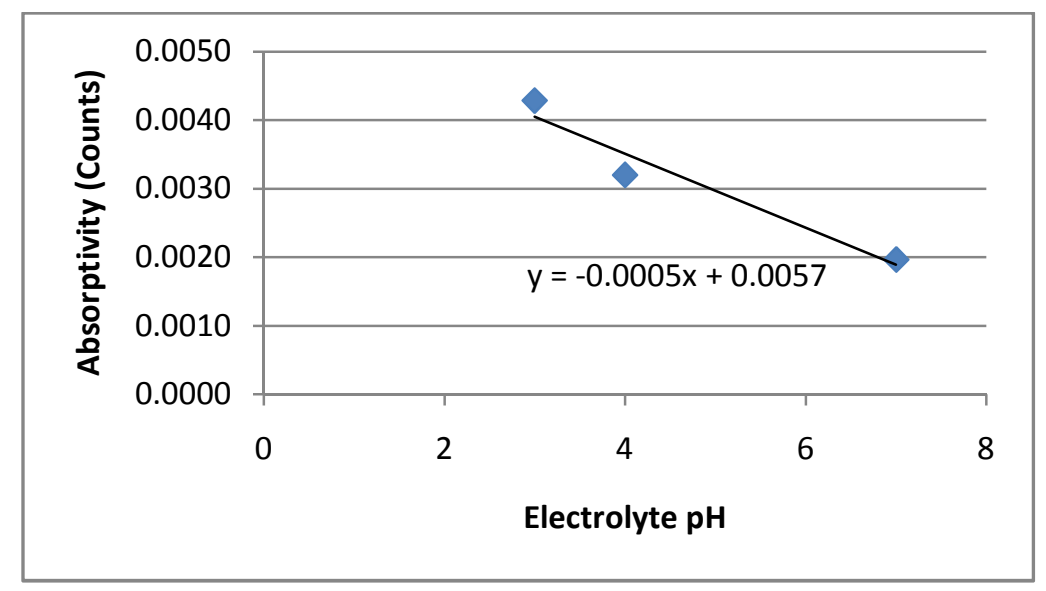

Figure 3.8: Cobalt molar absorptivity results as detected by the AAS. A trend towards elevated dissociation rates at lower $\mathrm{pH}$ was observed. 


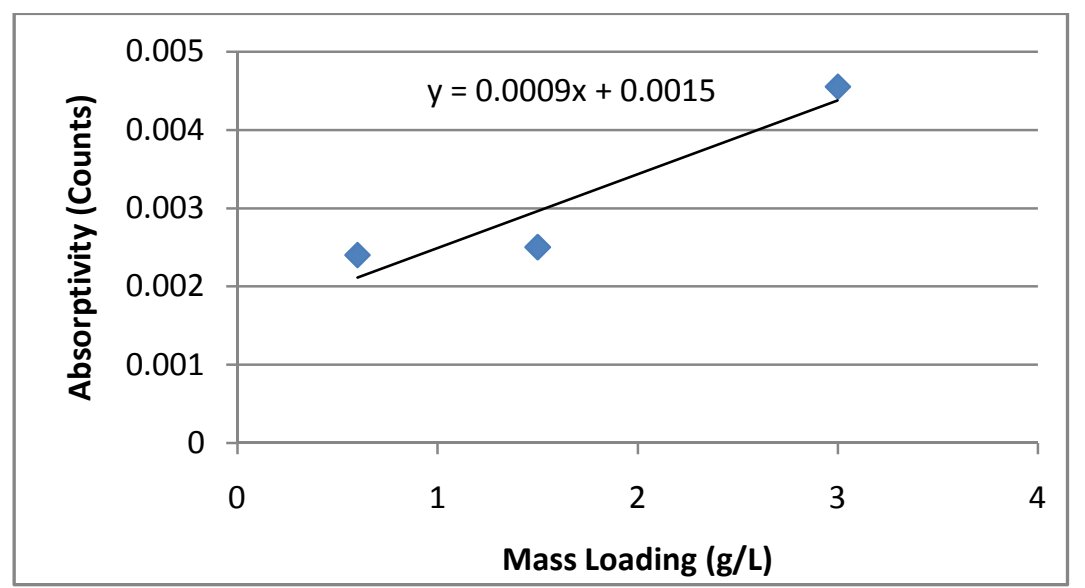

Figure 3.913: Cobalt molar absorptivity results as detected by the AAS. A trend towards elevated dissociation rates at higher mass loadings was observed.

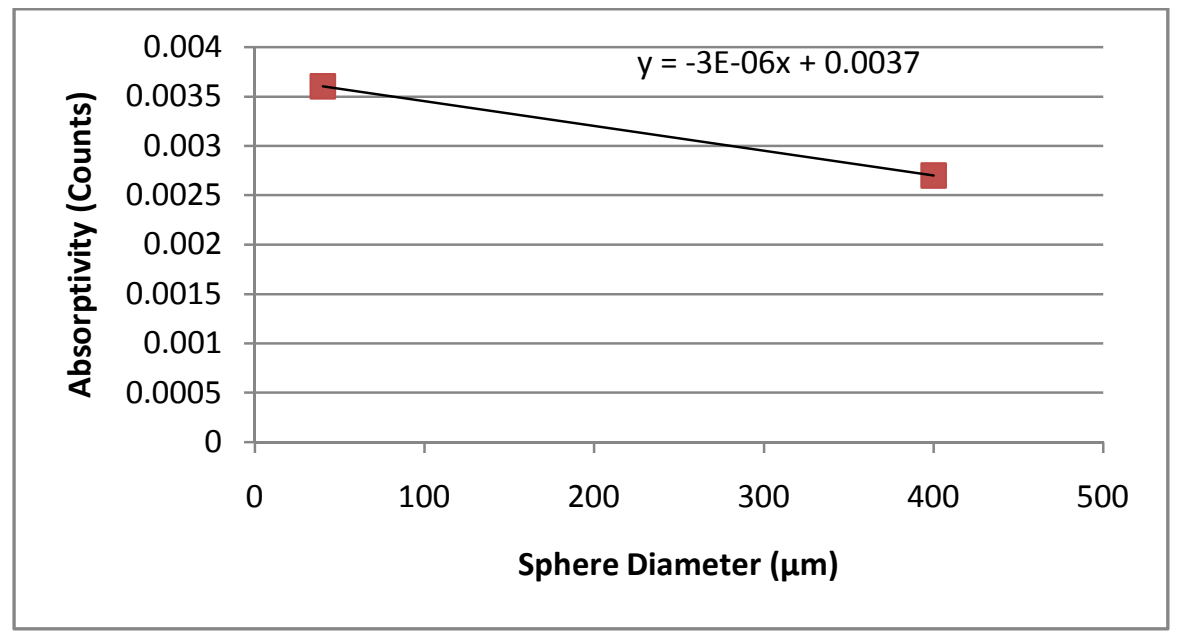

Figure 3.10: Cobalt molar absorptivity results as detected by the AAS. A trend towards elevated dissociation rates at smaller particle diameters was observed.

Not surprisingly, the data yielded by the third test indicated that in almost all cases, a $\mathrm{pH}$ of 3 would generate the highest ion concentration in the electrolyte (Figure 3.8). Additionally, higher loadings of particle weight in individual sample vials appeared to produce greater ion concentrations (Figure 3.9). A mass loading of $100 \mathrm{mg}$, or $3.0 \mathrm{~g} / \mathrm{L}$ generally had the highest ion concentration at the conclusion of the test period. Lastly, an encouraging trend of higher ion concentrations for smaller particle diameters was observed (Figure 3.10). 
Although the individual samples were not replicated enough to be considered significant, the observed trends were considered important enough to proceed in the direction indicated by the results. This direction was determined to be a study with a minimum of $2.0 \mathrm{~g} / \mathrm{L}$ of simulated wear debris immersed in solutions with lowered $\mathrm{pH}$ and faster agitation speeds coupled with longer test duration.

\subsubsection{EXPERIMENT 4: EXTENDED DURATION WITH HIGHER STATISTICAL POWER}

Based primarily on the results of the previous experiment, the fourth study performed was intended to have a significantly higher statistical power and to examine the influence of surface area on ion dissociation. The only variable of the experiment was particle diameter, with $400 \mu \mathrm{m}$ and $40 \mu \mathrm{m}$ being the only two levels.

Containment: $33 \mathrm{~mL}$ high density polyethylene (HDPE) vials were used for the containment of each individual sample.

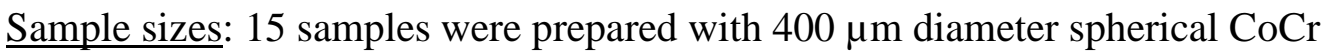
alloy particles and 15 samples were prepared with $40 \mu \mathrm{m}$ diameter spheres. Control samples with no particles were prepared with the deionized water used to rinse vials and the HBSS.

Loadings: The mass loading used was $100 \mathrm{mg}$ of the designated $\mathrm{CoCr}$ alloy sphere diameter. This equates to $3.0 \mathrm{~g} / \mathrm{L}$ of electrolyte.

Electrolyte: The HBSS used was modified with $\mathrm{HCl}$ to a $\mathrm{pH}$ of 3.4.

Test duration: 42 days

Agitation rate: $100 \mathrm{rpm}$

It is important to note that a strong argument can be made against the applicability of this experimental design to the in vivo conditions of the human body, as it is extremely 
rare for the body to ever reach a $\mathrm{pH}$ of 3.4; however the $\mathrm{pH}$ of very localized infection sites and hematomas do occasionally fall to this level in severe cases (Osborn, 1953). Regardless, the objective was to find consistent and conclusive evidence of ion dissociation and relate it to surface energy as a validation of the overall test methodology. With this objective, the reduction of $\mathrm{pH}$ to 3.4 is a logical step to accelerate the dissociation rate within reasonable parameters, particularly because theoretically, neither the dissociated ion species nor the oxide mechanics change at a slightly lower $\mathrm{pH}(\mathrm{Hsu}$, 2005).

The results of the fourth experiment (Figure 3.11) were significant in determining differences between the ion species analyzed as well as the debris diameters tested. As anticipated by the EDS results from the second experiment, cobalt ion concentrations in the electrolyte were significantly higher than chromium concentrations. The ion concentrations from the two levels of sphere diameter were also significantly different.

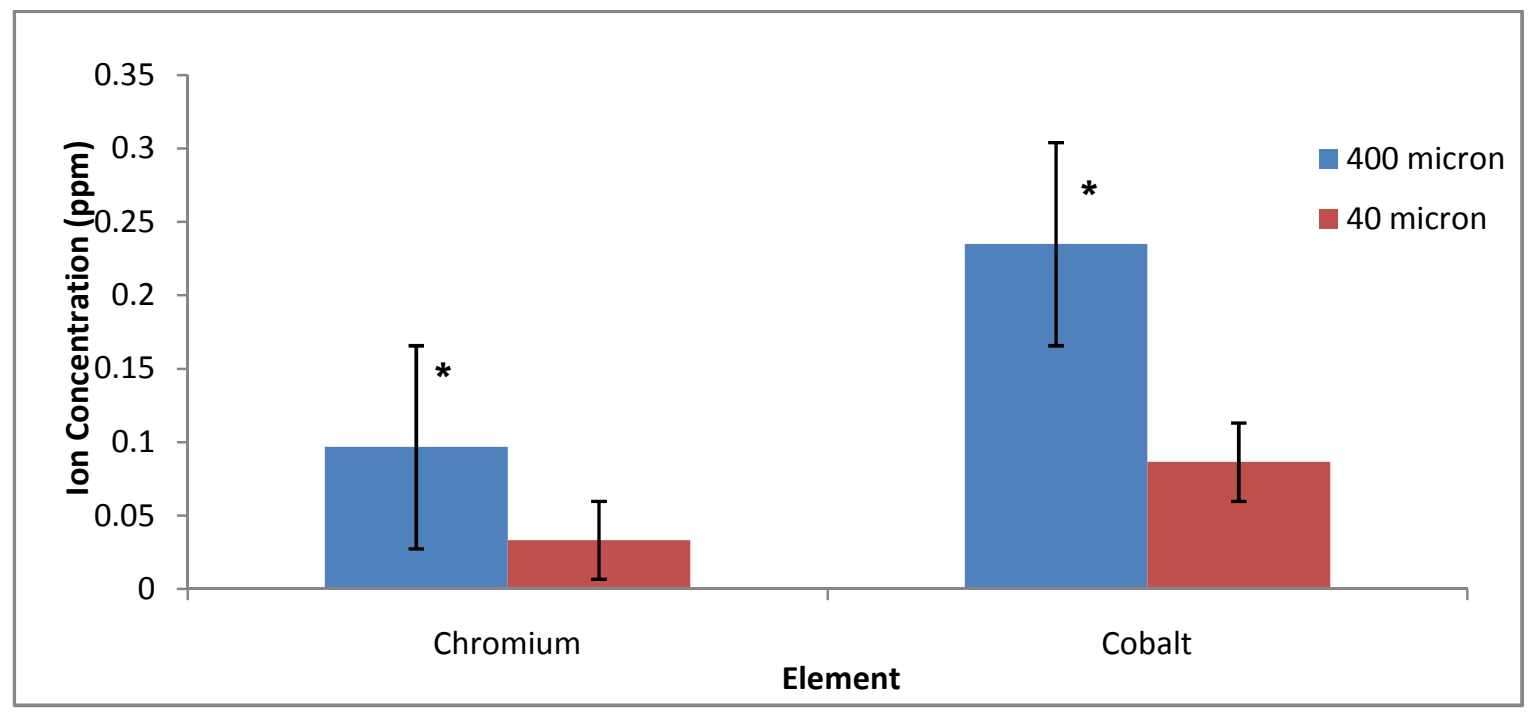

Figure 3.11: Graphical results from experiment 4. A statistically significant difference was detected between the diameters of the spheres tested as well as the comparative dissociation rates of cobalt and chromium for each diameter level. 
Unexpectedly, the $400 \mu \mathrm{m}$ diameter sphere dissociation rates were significantly higher than those of the $40 \mu \mathrm{m}$ diameter sphere dissociation rates. The mean chromium ion concentration for the $400 \mu \mathrm{m}$ samples after 42 days of testing was $0.0967 \mathrm{ppm}$ and the mean cobalt ion concentration was $0.235 \mathrm{ppm}$. For the $40 \mu \mathrm{m}$ samples, the mean chromium ion concentration was $0.0333 \mathrm{ppm}$ and the mean cobalt ion concentration was $0.0865 \mathrm{ppm}$. Although all samples were statistically different than control samples, the controversial results yielded in the fourth experiment required additional interpretation.

Two theories for the depressed dissociation rate in the $40 \mu \mathrm{m}$ diameter samples were developed: (1) the relatively high particle density within the individual sample vials led to an agglomeration phenomenon that effectively reduced the total exposed surface area of the $40 \mu \mathrm{m}$ diameter spheres to a level below that of the $400 \mu \mathrm{m}$ sphere samples; or (2) the low $\mathrm{pH}$ led to the rapid development of an oxide layer of constant thickness on the sphere surfaces, greatly reducing the ratio of unoxidized $\mathrm{CoCr}$ to oxidized $\mathrm{CoCr}$ on the smaller spheres and thus reducing their ion dissociation rate. Agglomeration was selected as the most likely cause for the $40 \mu \mathrm{m}$ dissociation rate being lower than the 400 $\mu \mathrm{m}$ rate.

Agglomeration of debris at similar particle densities is discussed by Midander et al. in their work on dissociation of stainless steel debris in biological media (Midander, 2006), although much of their work is not performed with the level of agitation found in this experiment. The phenomenon of agglomeration occurs most frequently in samples containing particles of very small diameters, including at the $40 \mu \mathrm{m}$ level (Posner, Anderson, \& Alexander, 1954). This is largely because debris on this scale is very closely spaced when uniformly dispersed in solution at concentrations as high as $3 \mathrm{~g} / \mathrm{L}$ and such 
tight space leads to electrochemical attraction between individual particles (Figure 3.12).

These attractions are magnified by the small masses of the individual particles and can

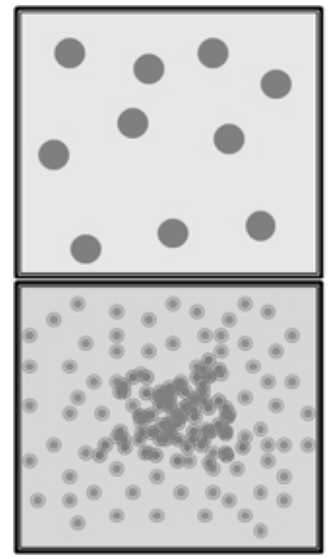

Figure 3.12: Representation of the effects of electrostatic forces on debris of decreasing diameter. potentially lead to the large-scale grouping of debris in such a way that overall surface area and thus dissociation rate can be drastically reduced. The dashed red line in Figure 3.13 illustrates the theoretical concept that agglomerated debris would effectively have less total exposed surface area than uniformly distributed debris, thus reducing the anticipated corrosion rate to a level consistent with larger diameter particles that remain evenly dispersed throughout the medium.

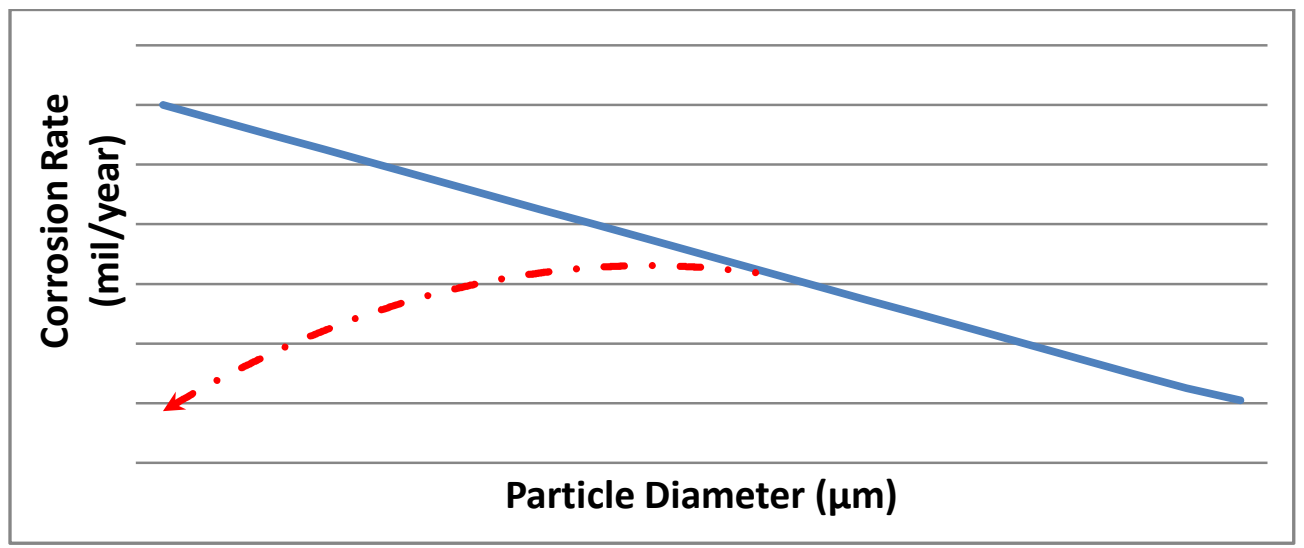

Figure 3.13: A representation of the effect of agglomeration (red line) on the expected particle corrosion rate (blue line). As debris reaches a critical size with a critical interparticle spacing, (which is related to the particle density in solution) the Van der Wals and other electrostatic forces between particles gain significance until they agglomerate. This agglomeration leads to a reduction in total exposed surface area and surface energy, which directly decreases the corrosion rate.

Further experimentation applied this theory of agglomeration by reducing the debris density in the electrolyte and increasing the agitation rate of the samples in order to maintain more spacing between particles.

\subsection{EXPERIMENT 5: HIGH STATISTICAL POWER WITH INCREASED LEVELS}


Following the unanticipated results of the previous test, the fifth and final experiment was targeted at improving the test methodology to produce reliable results in accordance with experimental objective number two and to evaluate the differences in dissociation rates at varying particle densities and varying $\mathrm{pH}$. Applying an understanding of agglomeration effects, the three levels for the particle density factor were $1.0 \mathrm{~g} / \mathrm{L}, 2.0 \mathrm{~g} / \mathrm{L}$, and $3.0 \mathrm{~g} / \mathrm{L}$. Four improvements were made to the methodology for the fifth experiment: (1) sterilized $22 \mathrm{~mL}$ glass vials were used for sample containment in order to reduce any possible interaction between the containment vial, (2) the test duration was lengthened to provide more relevant data, (3) the agitation rate was increased in an attempt to prevent agglomeration, and (4) the AAS ion detection system was replaced with a Thermo iCAP 6300 Inductively Coupled Plasma (ICP) Atomic Emission Spectrometer. The ICP detection system offers superior accuracy and detection limits as low as $10 \mathrm{ppb}$. The test conditions used in the fifth experiment were as follows:

Containment: $22 \mathrm{~mL}$ sterilized glass vials were used for the containment of each individual sample.

Sample sizes: 18 samples were prepared with $400 \mu \mathrm{m}$ diameter spherical $\mathrm{CoCr}$ alloy particles and 18 samples were prepared with $40 \mu \mathrm{m}$ diameter spheres. Control samples with no particles were prepared with the Hank's Balanced Salt Solution (HBSS) at each pH level.

Loadings: The levels of mass loading used were $22 \mathrm{mg}, 44 \mathrm{mg}$, and $66 \mathrm{mg}$ of the designated $\mathrm{CoCr}$ alloy sphere diameter. This equates to $1.0 \mathrm{~g} / \mathrm{L}, 2.0 \mathrm{~g} / \mathrm{L}$, and 3.0 $\mathrm{g} / \mathrm{L}$ in terms of particle density.

Electrolyte: The HBSS pH levels used were 3.4 and 7. 
Test duration: 50 days

Agitation rate: $140 \mathrm{rpm}$

\subsubsection{EXPERIMENT 5 RESULTS}

The ion concentrations detected in experiment five were statistically significant and generally representative of the original prediction that exposed surface area would play a role in the experiment corrosion kinetics. The enhancements to the methodology, particularly the use of the ICP and glass sample containers appeared to dramatically improve the consistency and precision of the test results. As test precision is a component of the research objectives, the use of this refined methodology for further testing is encouraged.

At $\mathrm{pH} 7$, the results of the cobalt ion concentration analysis positively correlated mass loading with ion concentration. Additionally, the electrolytes at each mass loading level carried a statistically greater ion concentration for the $40 \mu \mathrm{m}$ samples over the 400 $\mu \mathrm{m}$ samples (Figure 3.14). 


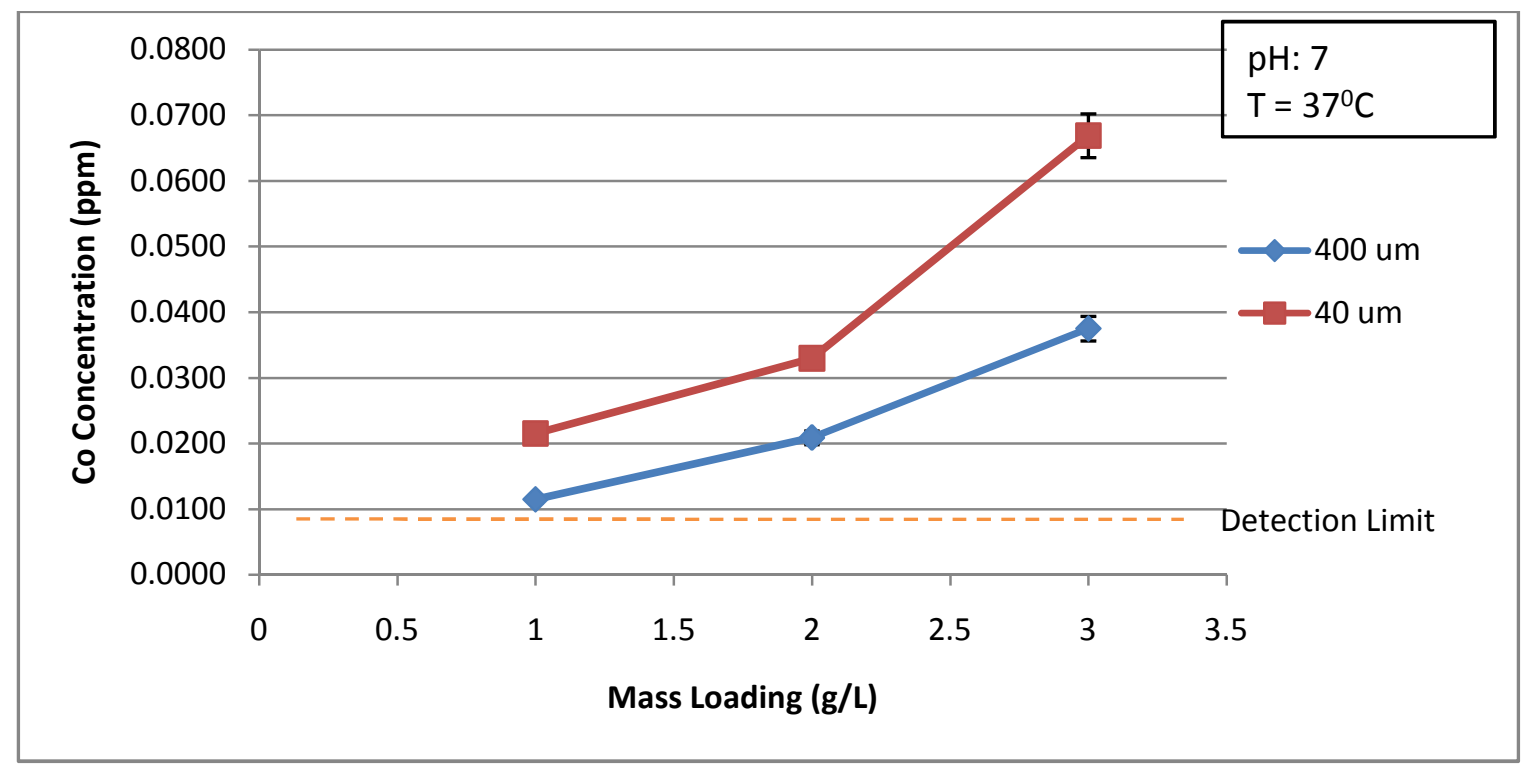

Figure 3.14: Detected cobalt concentrations at each mass loading level and for each sphere diameter. The significant trend of increasing concentration in relation to both increasing surface area to volume ratio and increasing mass loading can be discerned here.

Consistent with the EDS results from experiment two, the chromium ion concentrations in the $\mathrm{pH} 7$ samples were not significantly elevated over the controls, implying that longer test durations would likely be required to acquire sufficient distinguishable concentrations of chromium ions (Figure 3.15). Alternatively, in an attempt to improve the detection of chromium ions, the electrolytic solutions used could potentially be replaced with a phosphate buffered saline or other biologically similar fluid, as the calcium content of HBSS likely absorbs a great deal of plasma energy during ICP analysis. This high rate of energy absorption effectively diminishes the detection of less emissive ions, such as chromium. 


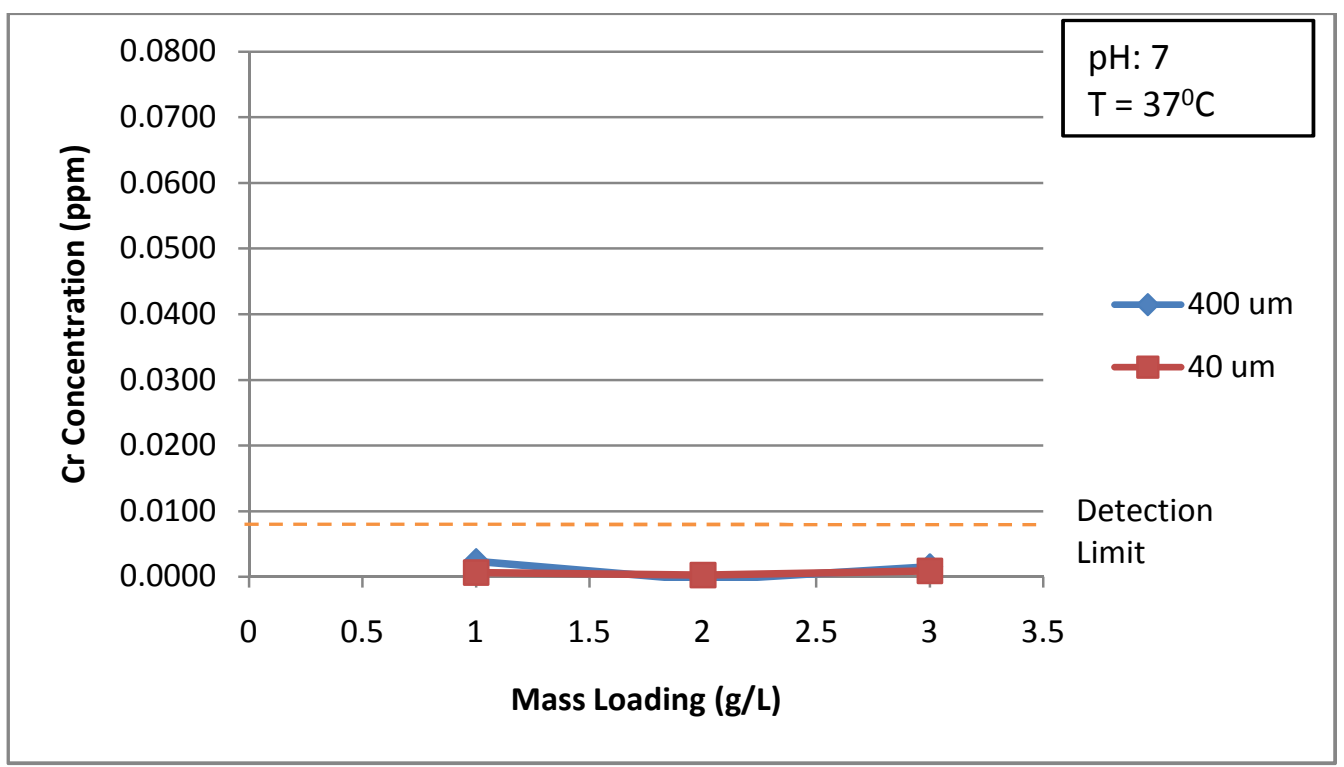

Figure 3.15: Extremely low concentrations of chromium were detected, leading to the conclusion that negligible chromium dissolution occurred during the test period, as all results were below the theoretical detection limit.

An ANOVA interactions plot was prepared for the $\mathrm{Co}$ and $\mathrm{Cr}$ ion concentrations in order to identify any data anomalies resulting from factor interaction. As seen in

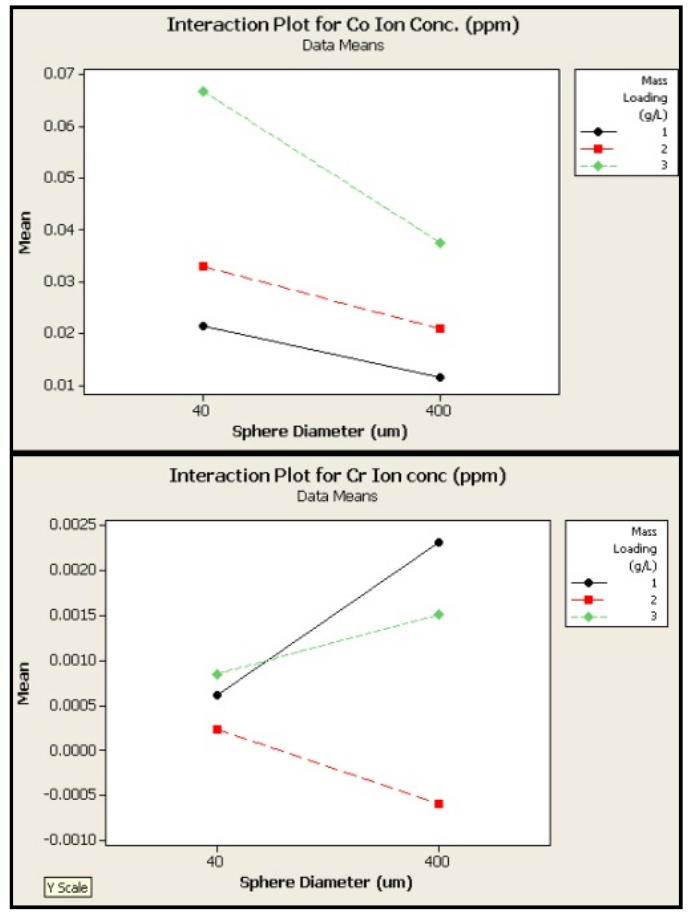

Figure 3.16: An interactions analysis of $\mathrm{Co}$ and $\mathrm{Cr}$ concentrations at each mass loading and sphere diameter level for $\mathrm{pH} 7$ samples. No significant interactions were detected. The potential interaction seen in the $\mathrm{Cr}$ graph was determined to be negligible
Figure 3.16, no interactions were detected for

the cobalt results and the potential interactions

seen for the chromium results were considered

negligible, considering the inconclusive

chromium detection levels. 
The results of the ICP analysis for the $\mathrm{pH} 3$ samples were less conclusive than those of the $\mathrm{pH} 7$ samples, presenting new interpretation challenges. The results for the ion analysis at the $\mathrm{pH} 3$ level were statistically higher than control samples for both cobalt and chromium ion concentrations. Additionally, chromium ions were less prevalent than cobalt ions, which was anticipated. The electrolytes from those $\mathrm{pH} 3$ samples containing $400 \mu \mathrm{m}$ diameter particles had statistically higher ion concentrations for both cobalt (Figure 3.17) and chromium (Figure 3.18) than did the electrolytes containing $40 \mu \mathrm{m}$ spheres, which represented the opposite trend seen in the $\mathrm{pH} 7$ analysis. The results of the $\mathrm{pH} 3$ data set also showed a less decisive correlation between mass loading and ion concentration, with both the chromium and cobalt results showing inconclusive trends between mass loadings at the $40 \mu \mathrm{m}$ level.

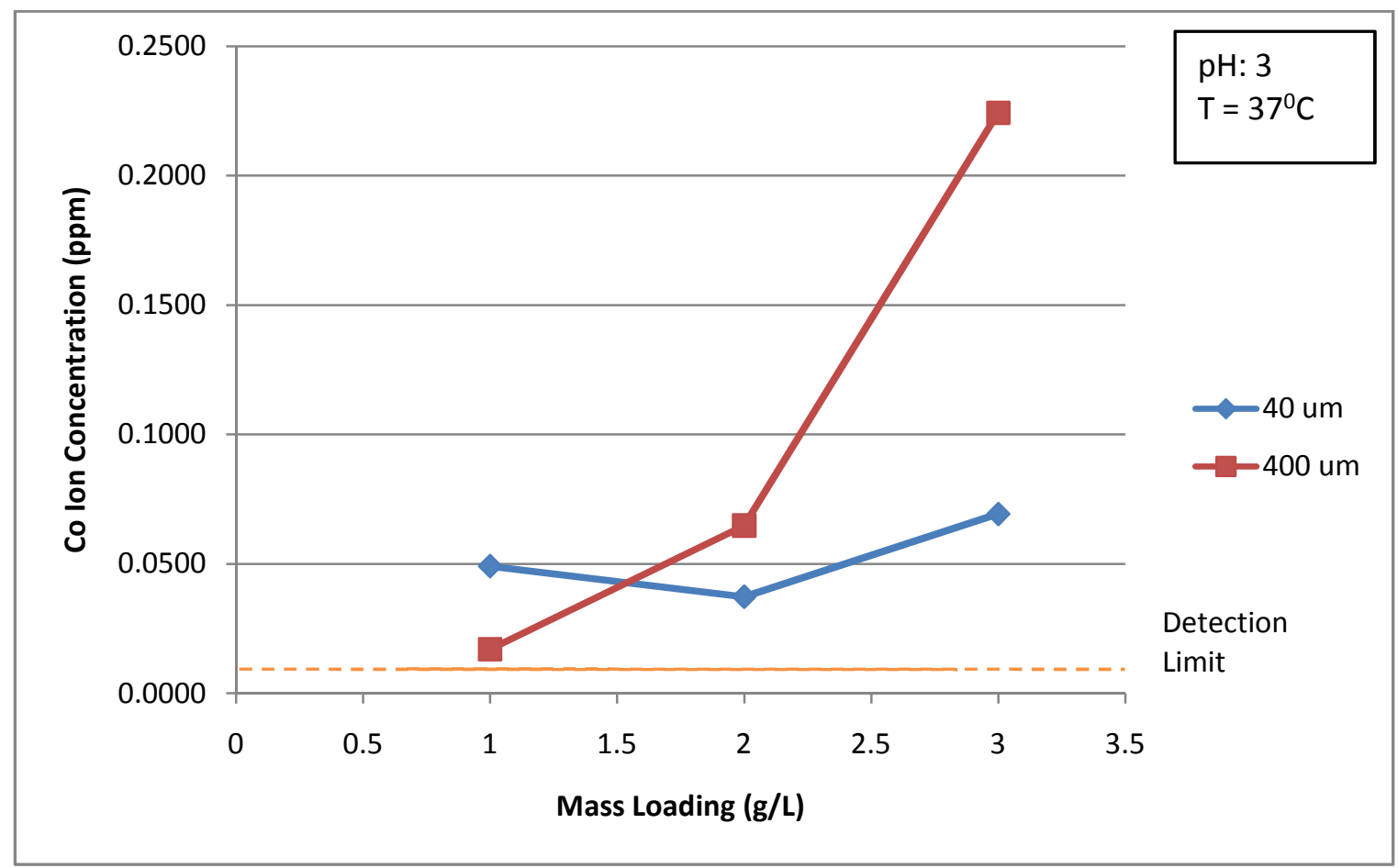

Figure 3.17: Co ion concentrations detected at the $\mathrm{pH} 3$ level. Potential agglomeration effects can be seen in the decreased ion concentrations at the $40 \mu \mathrm{m}$ level. 


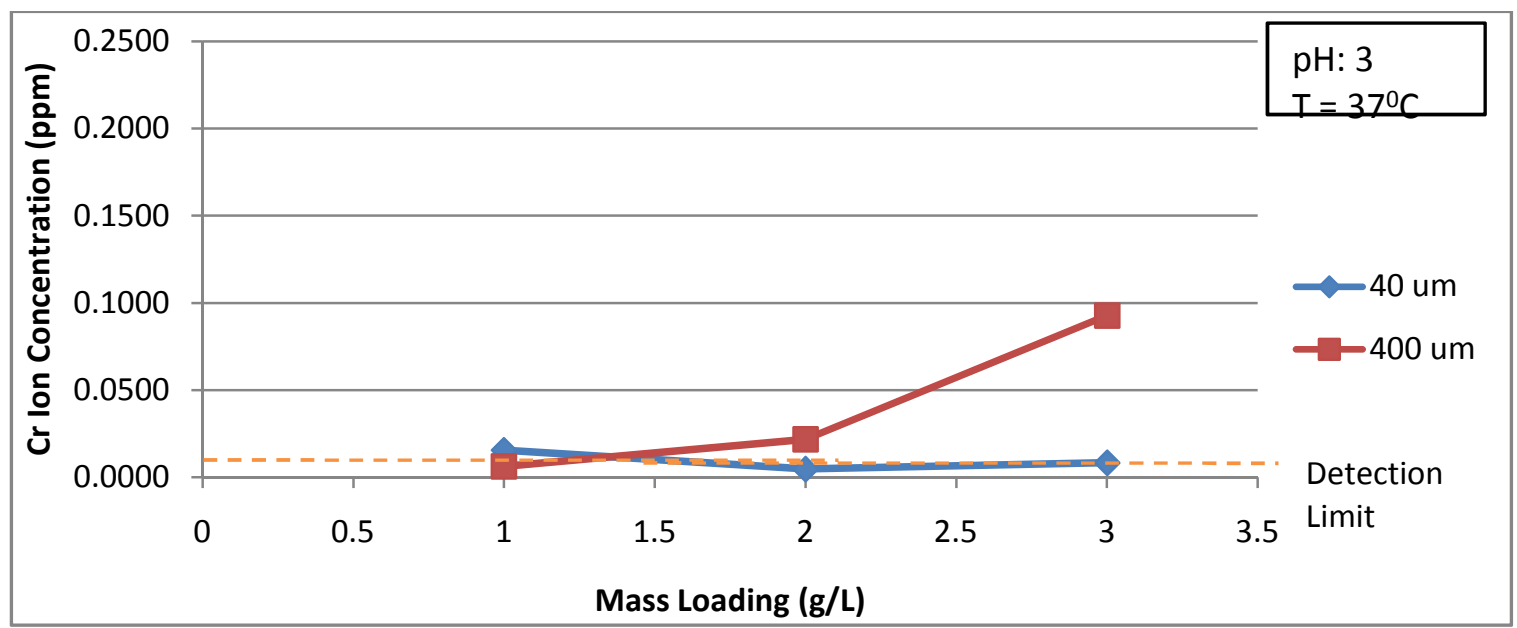

Figure 3.18: $\mathrm{Cr}$ ion concentrations detected at the $\mathrm{pH} 3$ level. Potential agglomeration effects can be seen in the decreased ion concentrations at the $40 \mu \mathrm{m}$ level.

A second set of interactions plots (Figure 3.19) was prepared to compare the data collected at $\mathrm{pH} 3$ and to identify interactions between the different $\mathrm{pHs}$. Strong evidence of an interaction exists between $\mathrm{pH}$ and sphere diameter as well as between mass loading and sphere diameter at $\mathrm{pH}$ 3. These interactions suggest that, contrary to previously discussed literature, the ion dissociation rates of the $\mathrm{pH} 3$ samples are in some way affected differently than the $\mathrm{pH} 7$ samples. 


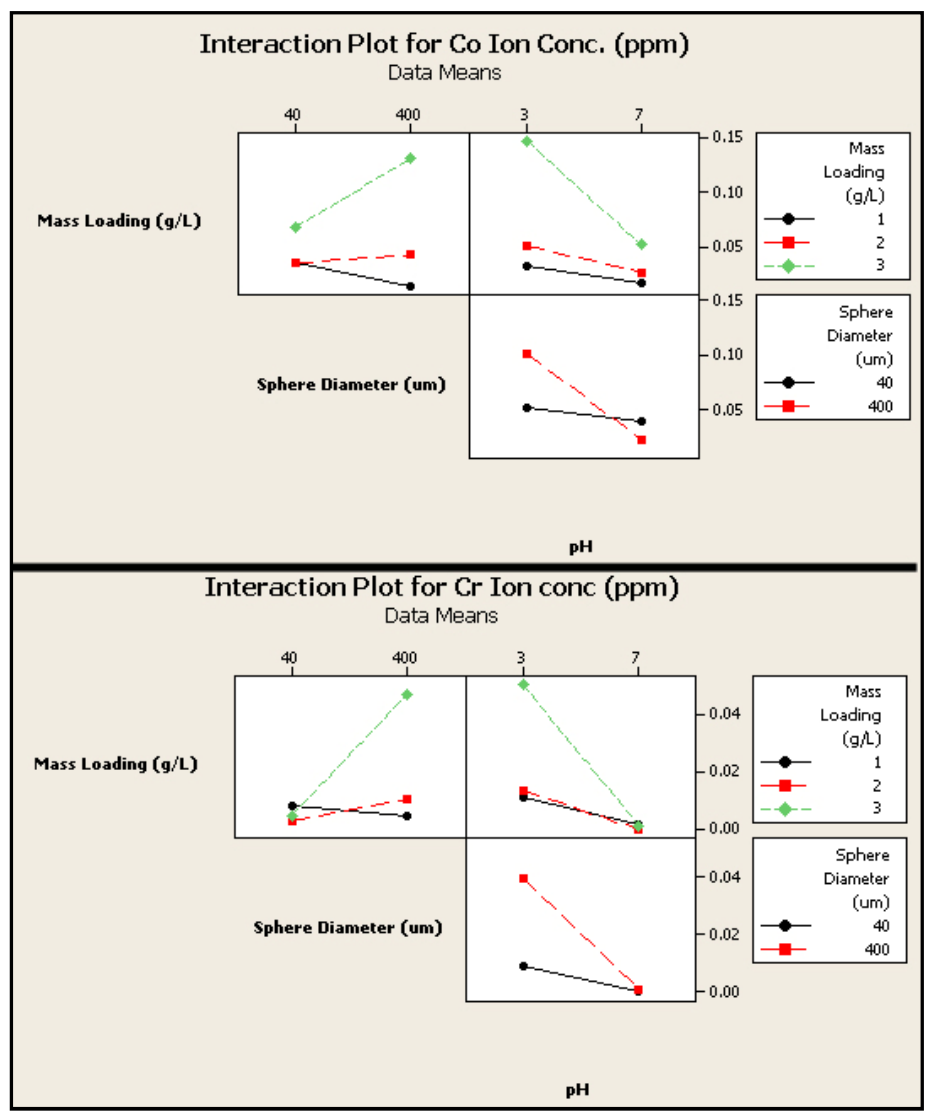

Figure 3.19: An interactions analysis of Co and $\mathrm{Cr}$ concentrations at each mass loading, pH, and sphere diameter level. Strong evidence of an interaction exists between $\mathrm{pH}$ and sphere diameter as well as between mass loading and sphere diameter at $\mathrm{pH} 3$.

The differing ion concentration trends at the $\mathrm{pH} 3$ level suggest several possibilities for the depressed ion concentrations for $40 \mu \mathrm{m}$ samples. Because similar results were obtained in a statistically significant manner during the fourth experiment, it can be concluded that the lowered $\mathrm{pH}$ artificially decreases the ion dissociation rate dependence on surface area for CoCr spheres in HBSS, contrary to the original estimation that only corrosion kinetics would vary in decreasing $\mathrm{pH}$ solutions. Isolating this source of counter-intuitive results leads to the conclusion that a $\mathrm{pH}$ depressed solution potentially leads to increased viscosity or an electrochemical attraction between debris. Either of these possibilities could lead to an increase in the effect of agglomeration phenomena discussed in section 3.4.4, which would correlate to a reduction in overall 
surface area to volume ratio, a depression of surface energy below that of the $400 \mu \mathrm{m}$ samples, and thus a decline in the ion dissociation rate for the $40 \mu \mathrm{m}$ diameter bearing samples. Another possibility for the decrease in $40 \mu \mathrm{m}$ sample dissociation rate is the rapid formation of a thicker oxide than that which occurred on the $40 \mu \mathrm{m}$ samples at $\mathrm{pH}$ 7. The rapid formation of such an oxide would potentially increase the ratio of the oxide thickness volume to the unoxidized volume of the sample in such a way that the mass transport regulated system of ion dissociation would be affected only for the smaller diameter samples, because the ratio difference for the larger samples would remain negligible. This theory was evaluated using a Focused Ion Beam (FIB) and EDS depth analysis, the results of which are discussed in section 3.6.

\subsubsection{EXPERIMENT 5 DISCUSSION}

\section{PREDICTING COCR DISSOLUTION RATES}

Little research has been published regarding surface area dependence for modeling CoCr corrosion rates. According to a 1995 study by Browne et al. in one of the few published works on the topic, the dissolution rate of orthopaedic wear debris is directly related to the total exposed surface area, which is in turn related to individual particle diameter. Browne et al. suggest that the relationship between surface area and corrosion rate is linear for very small particle diameters and can be calculated as $\left[\mathrm{Cr}^{+2}\right]=3.387 * S A+0.318$, where $\left[\mathrm{Cr}^{+2}\right]$ is the chromium ion concentration $(\mu \mathrm{g})$ after 24 hours and $S A$ is the total surface area of the particles in solution $\left(\times 10^{10}\right)$. Without evidence outside this experiment to suggest otherwise, this thesis will assume equivalent dissolution rates for cobalt and chromium ions. Using this equation and both 
geometric and mass data (Table 3.4) regarding the representative particles used, the predicted ion dissolution rate was calculated for the particles in this experiment.

\begin{tabular}{|c|c|c|}
\hline Physical Features & $40 \mu \mathrm{m}$ Spheres & $400 \mu \mathrm{m}$ Spheres \\
\hline Mass & $0.00028 \mathrm{mg}$ & $.278 \mathrm{mg}$ \\
\hline Surface Area & $5,030 \mu \mathrm{m}^{2}$ & $503000 \mu \mathrm{m}^{2}$ \\
\hline Volume & $33,510 \mu \mathrm{m}^{3}$ & $33,500,000 \mu \mathrm{m}^{3}$ \\
\hline
\end{tabular}

Because each mass loading was assumed to be $3 \mathrm{~g} / \mathrm{L}$, or $66 \mathrm{mg}$ in a $22 \mathrm{~mL}$

sample container, the number of particles in each sample, $N$, was calculated using this mass:

$N=66 \mathrm{mg} / \mathrm{m}$, Where $m$ is the mass of each individual sphere.

$$
\begin{aligned}
& N_{400 \mu m}=\frac{66 \mathrm{mg}}{.278 \mathrm{mg}}=237 \text { spheres } / \text { sample } \\
& N_{40 \mu \mathrm{m}}=\frac{66 \mathrm{mg}}{0.00028 \mathrm{mg}}=238,000 \text { spheres } / \text { sample }
\end{aligned}
$$

The total surface area for each sample was calculated based on the assumption that each individual CoCr sphere would be completely isolated from other spheres. While this assumption was clearly approximate in a test with a particle density of $3 \mathrm{~g} / \mathrm{L}$, it was assumed to be offset by the slightly nonuniform surface of the individual spheres (Figure 3.20), which had microscopic trenches and voids at the dendrite boundaries. These microscopic variations would increase the actual surface area over the calculated, while contact between the spheres would in turn reduce the total surface area to an approximately nominal value. Based on the number of spheres $(\mathrm{N})$ within a test sample, the total surface area was calculated for each sample diameter as follows: 
$119,000,000$

$1,190,000,000$

Based on the above calculations of total exposed surface area in each loaded

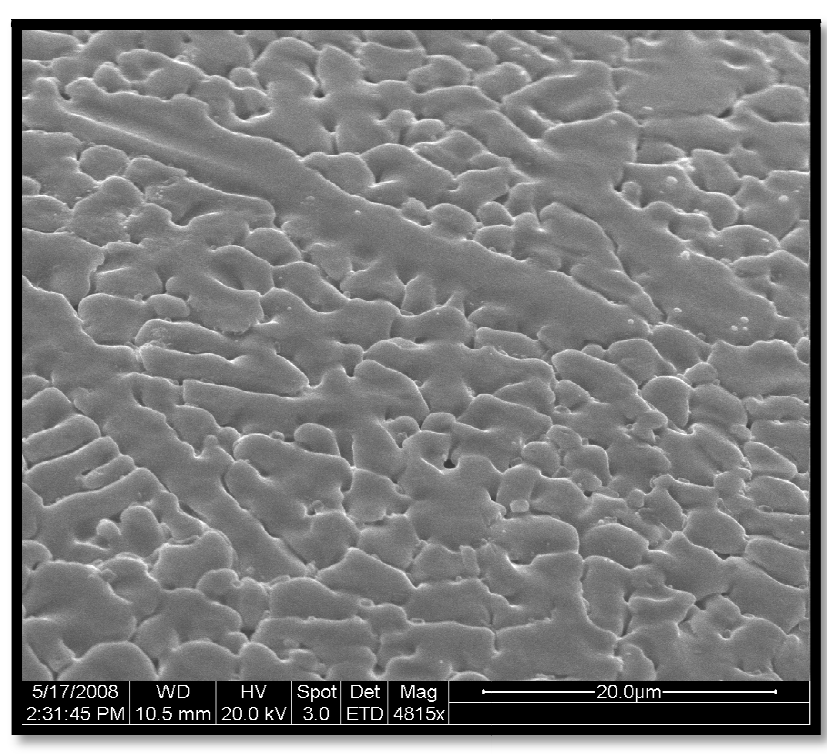

Figure 3.20: The dendritic structure of the spherical ASTM F75 CoCr alloy particles used to simulate wear debris . experimental sample, the anticipated corrosion rate was calculated using extrapolated information (Figure 3.21) from the prior works of Browne et al., Contu et al., and Metikos-Hukovi et al. Using the linear relationship discovered by Browne et al. for the chromium dissolution rate in inorganic buffered saline solution, (

$$
\text { , the daily ion weight }
$$

release rate could be estimated as $0.772 \mu \mathrm{g} /$ day and $0.359 \mu \mathrm{g} /$ day for the $40 \mu \mathrm{m}$ and 400 $\mu \mathrm{m}$ samples respectively. Over 42 days of the typical experiment discussed in this paper, this translates to $30.34 \mu \mathrm{g}$ of dissociated ions in the $40 \mu \mathrm{m}$ samples and $15.07 \mu \mathrm{g}$ of dissociated ions in the $400 \mu \mathrm{m}$ samples, which is equivalent to approximately $1.38 \mathrm{ppm}$ and $0.685 \mathrm{ppm}$ in a $22 \mathrm{~mL}$ sample respectively, assuming that the electrolyte is the density of water $\left(1 \mathrm{~g} / \mathrm{mL}^{3}\right)$. 


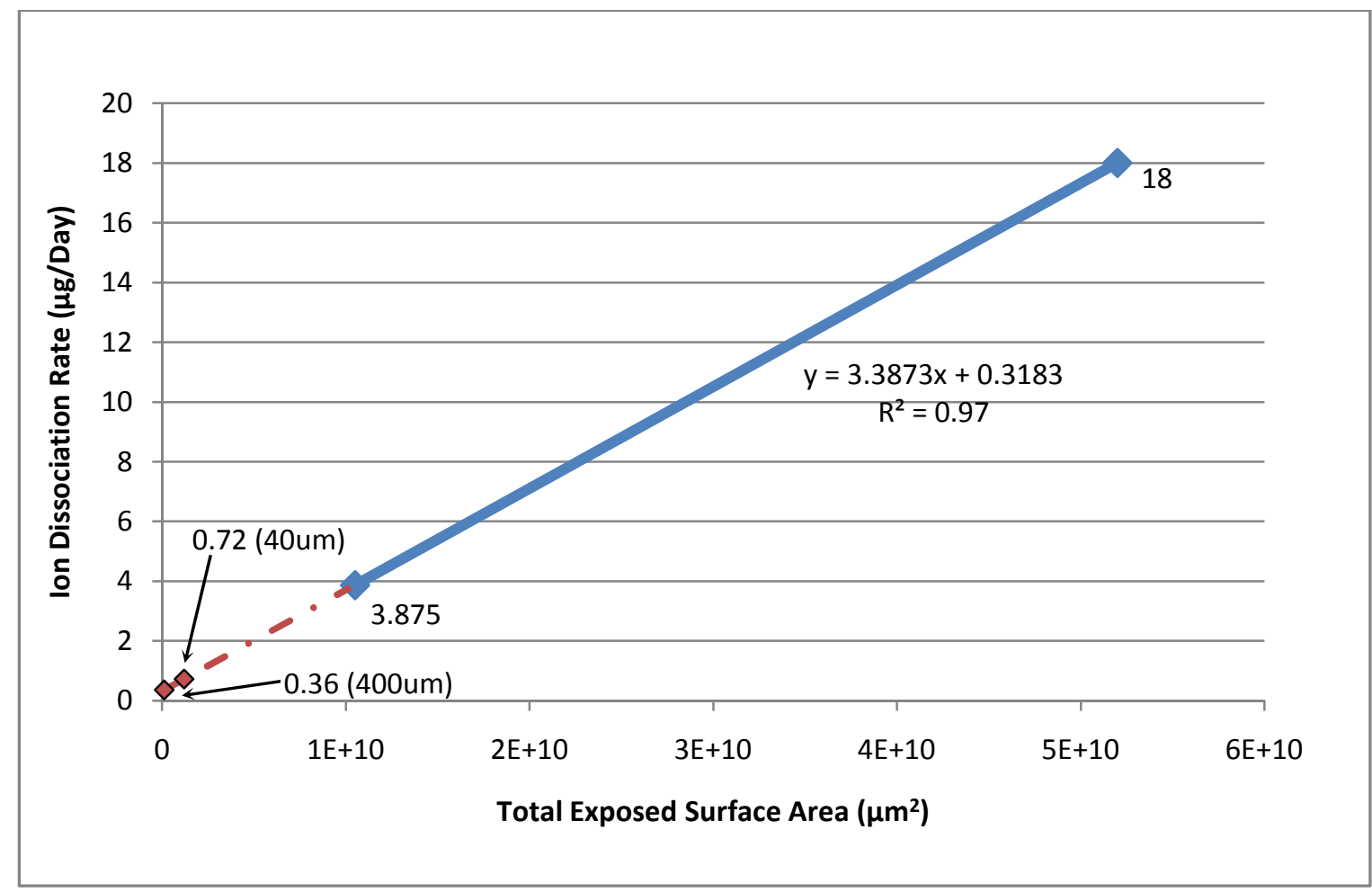

Figure 3.21: Estimated dissociation mass (ug) for micro-scale wear debris. Browne's linear fit curve (blue line) was extrapolated to $0.005 \times \mathrm{E} 10 \mu \mathrm{m} 2$ (red line) in order to include the large-diameter samples with low total exposed surface areas used in this experiment (Browne \& Gregson, 1995).

It should be noted that the work of Browne et al. applies only to small particle diameters $(2.6 \mu \mathrm{m}$ and $4.6 \mu \mathrm{m})$ and the linear extrapolation from their previous data will likely overestimate the dissociation rate for $400 \mu \mathrm{m}$ diameter spheres. This is for two reasons: 1) the extrapolation of data two orders of magnitude outside its original limits is not likely to yield accurate results, and 2) it is unlikely that dissociation rate depends simply on total exposed surface area.

Rather than depending on only the total exposed surface area, but on the total debris surface area to volume ratio, or surface energy, which changes drastically faster than a linear relationship would suggest. This is because as the diameter of an individual wear particle decreases, its surface area decreases at a slower rate than its volume, as the radius term in the volume equation is cubed. As can be seen in Figure 3.22, the surface 
area term in the surface area to volume relationship will theoretically become dramatically more pronounced at particle diameters below $50 \mu \mathrm{m}$.

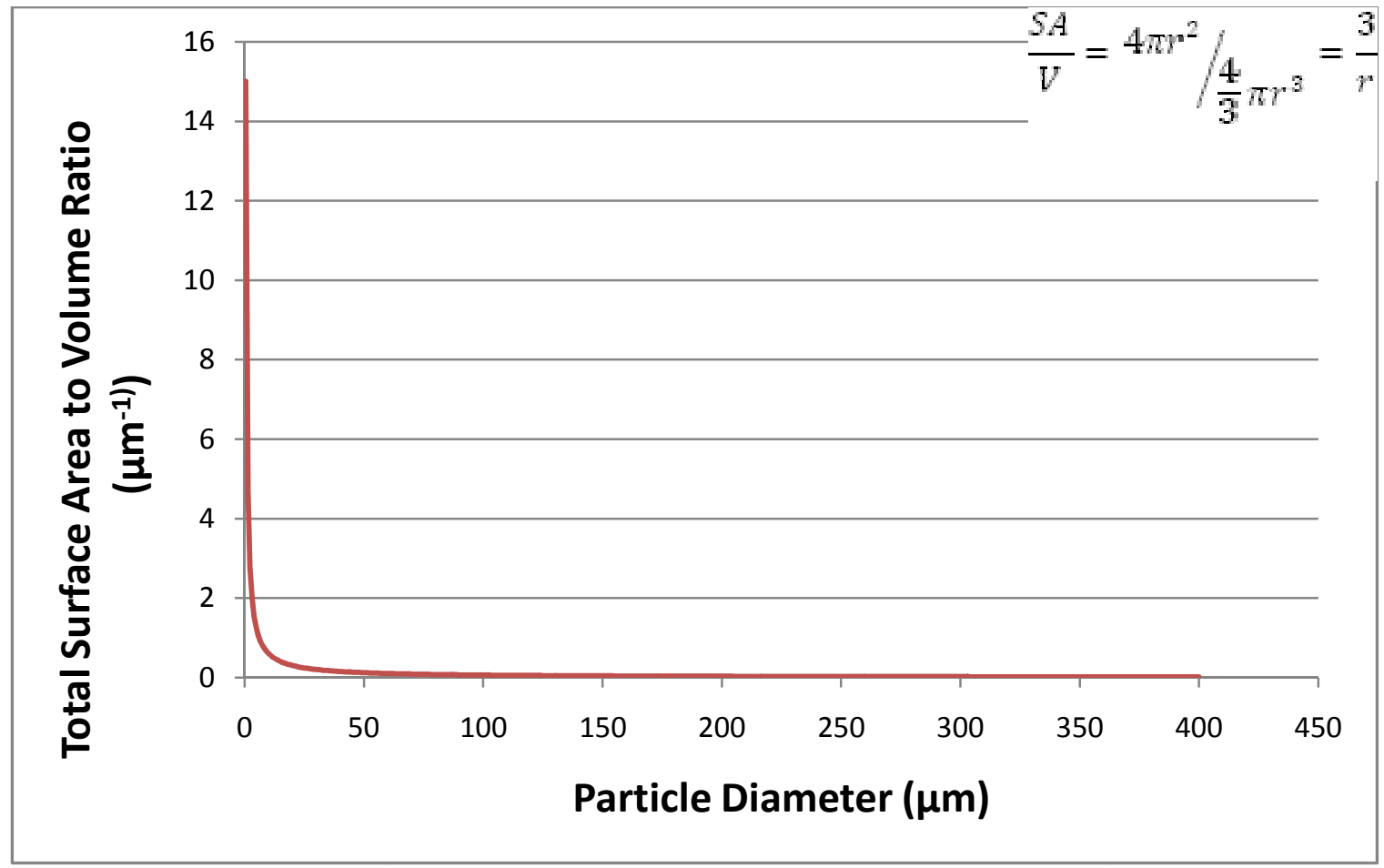

Figure3.22: For particulate debris samples, the theoretical surface area to volume ratio rises dramatically at particle diameters below $50 \mu \mathrm{m}$. This represents an increase in the relevance of the surface area term.

At such particle diameters as those used by Browne et al. and those present in true orthopaedic wear systems, the surface energy relationship is drastically higher than for the large particle diameters used in this test. This suggests a rapidly accelerating dissociation rate for particle diameters below $50 \mu \mathrm{m}$. Given this predicted increase in dissociation rate for the samples used by Browne et al., it is unlikely that the simple linear relationship predicted by Browne et al. will extend to the large diameter spheres used in this thesis. In spite of this, Browne et al.'s linear relationship between total exposed surface area and dissociation rate provides a basic ion dissociation rate for comparison. 


\section{COMPARISON OF DATA TO PREDICTED RESULTS}

Based on the 1995 model presented by Browne et al., the predicted dissociated ion mass for cobalt ions from a $40 \mu \mathrm{m}$ diameter sphere after 42 days of immersion in $\mathrm{pH}$ 7 HBSS electrolyte was $30.34 \mu \mathrm{g}$, or $1.38 \mathrm{ppm}$ in $22 \mathrm{~mL}$ of solution, assuming a $3 \mathrm{~g} / \mathrm{L}$ particle density. The actual detected Co ion concentration for identical circumstances was $0.0669 \mathrm{ppm}$. This result is considered to be statistically less than the predicted value, although it is not unexpected, as the linear extrapolation of Browne et al.'s results was anticipated to yield a high estimate for the reasons listed in the previous section. The $0.0375 \mathrm{ppm}$ detected in the $400 \mu \mathrm{m}$ sample of identical conditions (42 days, $\mathrm{pH} 7$ ) follows the anticipated trend of decreasing ion dissociation rate with increasing sphere diameter. While these values are not considered to be close to the predicted ion concentrations, they do reflect the same trend of a two fold increase in ion concentration between $40 \mu \mathrm{m}$ and $400 \mu \mathrm{m}$ diameter samples. In order to illustrate the differences in the predicted and actual dissociation rates, Figure 3.23 compares the predicted Co ion concentrations with the actual results previously discussed. Chromium results were not compared, due to their being below the ICP detection limit. 


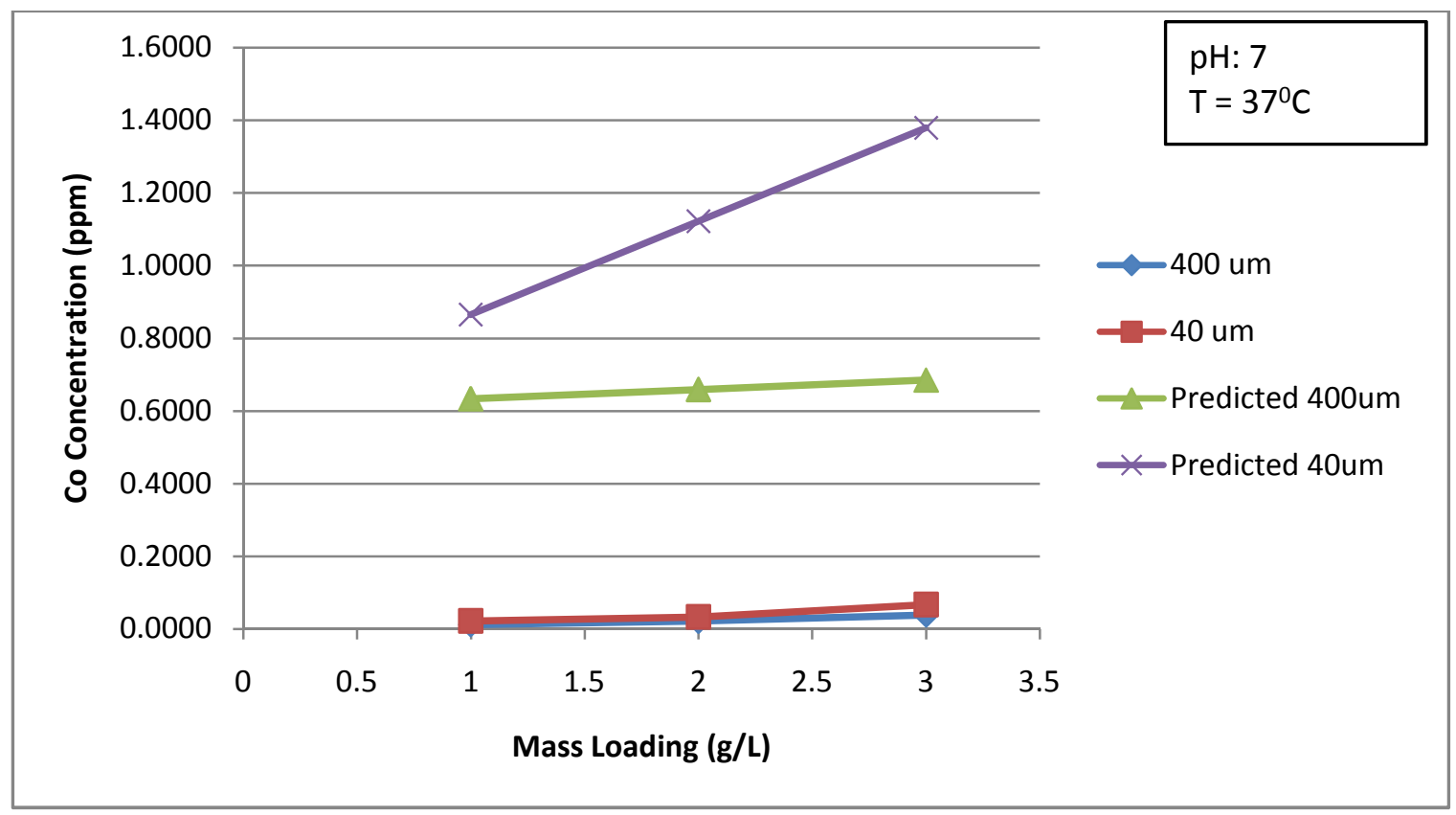

Figure3.23: The predicted ion concentrations for $400 \mu \mathrm{m}$ and $40 \mu \mathrm{m}$ particles compared to the actual results.

While the ion detection results for cobalt were significant, the prediction for specifically chromium dissolution was not considered to accurately represent the $\mathrm{Cr}$ dissolution in this experiment, largely due to undetectable $\mathrm{Cr}$ ion concentrations in the electrolytes. The lack of Cr dissolution was confirmed through EDS analysis, although further research is required before this result is applied to in vivo dissociation characteristics.

\section{EXTRAPOLATING RESUlTS TO TRUE WEAR DEBRIS}

The ion concentration results of the $3 \mathrm{~g} / \mathrm{L}$ mass loading level of experiment five were plotted and analyzed for dependence on surface area to volume ratio. While this study is targeting the relationship between ion dissociation rate and particle surface area to volume ratio, for ease of interpretation and applicability to future work, the plot was prepared with only the particle diameter on the $\mathrm{x}$-axis and the dissociation rate on the $\mathrm{y}$ axis. The particle diameter, $\mathrm{D}$, is directly related to its surface area to volume ratio 
through the relationship $\frac{S A}{V}=6 * D^{-1}$ (Figure 3.22). This relationship dictated the use of a power fit curve for the two tested particle diameters, rather than the simple linear fit used by Browne et al. Figure 3.24 shows this fit curve with only the data collected at $\mathrm{pH}$ 7 and at the $3 \mathrm{~g} / \mathrm{L}$ loading level.

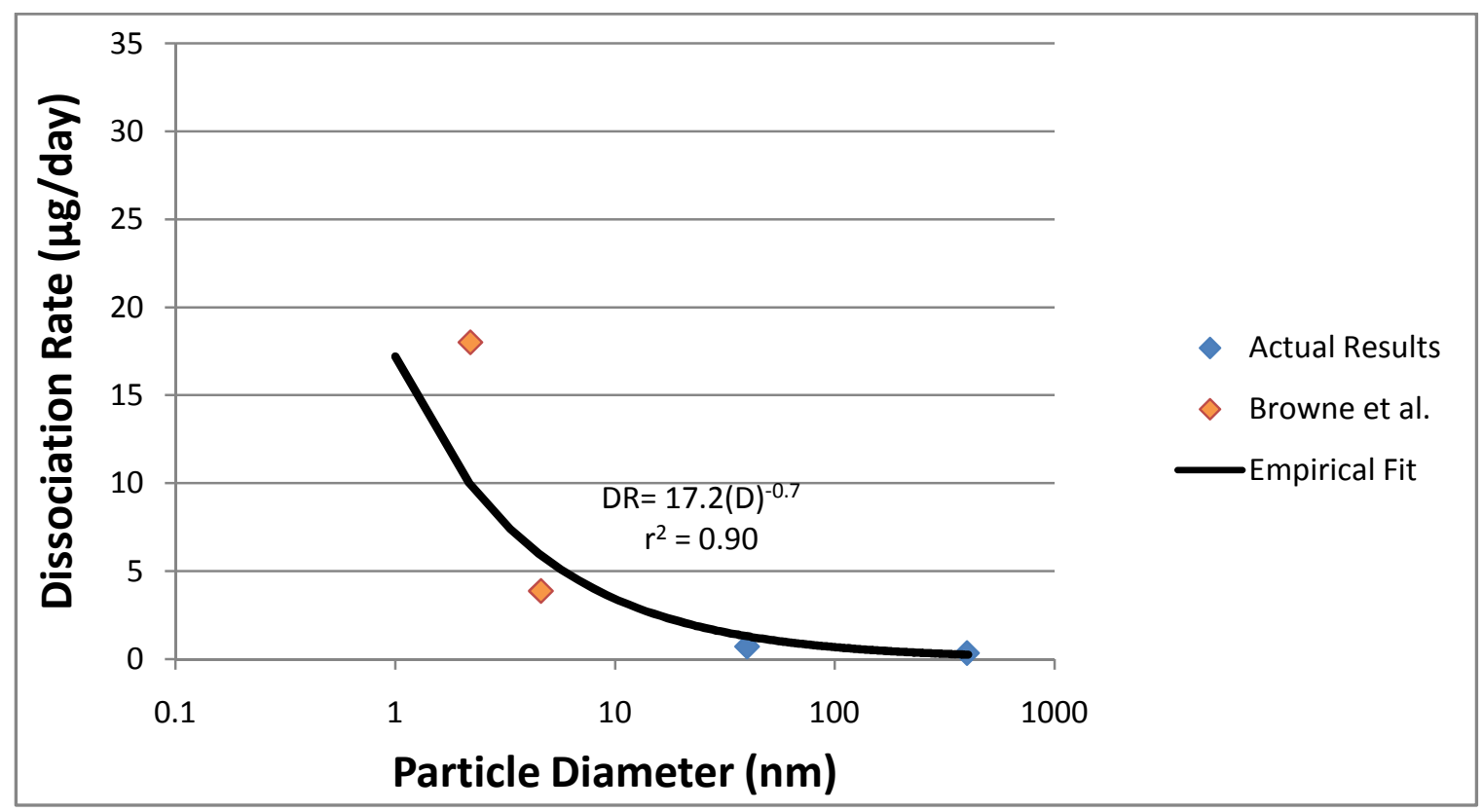

Figure3.24: Plot of results of experiment 5 and the fit curve used to extrapolate data to true wear debris, based on knowledge of the significance of the surface area term in the surface area to volume ratio at particle diameters below $40 \mu \mathrm{m}$.

The curve developed in this thesis is an improvement on the previous linear relationship used to predict the dissociation rate of varied debris diameters, because it accommodates both data sets and accounts for the dramatic differences in the surface area to volume ratio that take place at such small particle sizes. The particular power curve used in this empirical fit differs slightly from the $\frac{S A}{V}=6 * D^{-1}$ curve seen in the relationship between surface area and volume. This difference is likely due to the influence of rate limiting factors outside the thermodynamic factor in dissociation rate. These factors likely include oxide development and biological surface effects. 
The fit curve used to characterize both the data from Browne et al. as well as the experimental results from this experiment was then also extrapolated to include dissociation rates for $100 \mathrm{mg}$ samples of $400 \mathrm{~nm}$ diameter particles, which are on the order of true wear debris, as shown in Figure 3.25. While this extrapolation incorporates numerous assumptions, including steady state dissociation, ideal spherical shape, a constant particle surface area during dissolution, and negligible oxide effects, it is an introductory correlation between particle diameter and dissociation rate. The relationship between particle diameter, $\mathrm{D}$, which inherently incorporates the dependence on surface area to volume ratio, and ion dissociation rate, $\mathrm{DR}$, was found to be $D R=17.2 * D^{-0.7}$, where DR is measured in $\mu \mathrm{g} /$ day and $\mathrm{D}$ is given in nanometers. The extrapolated dissolution rate yielded for a $100 \mathrm{mg}$ sample containing $400 \mathrm{~nm}$ diameter spheres is 32.7 $\mu \mathrm{g} / \mathrm{day}$.

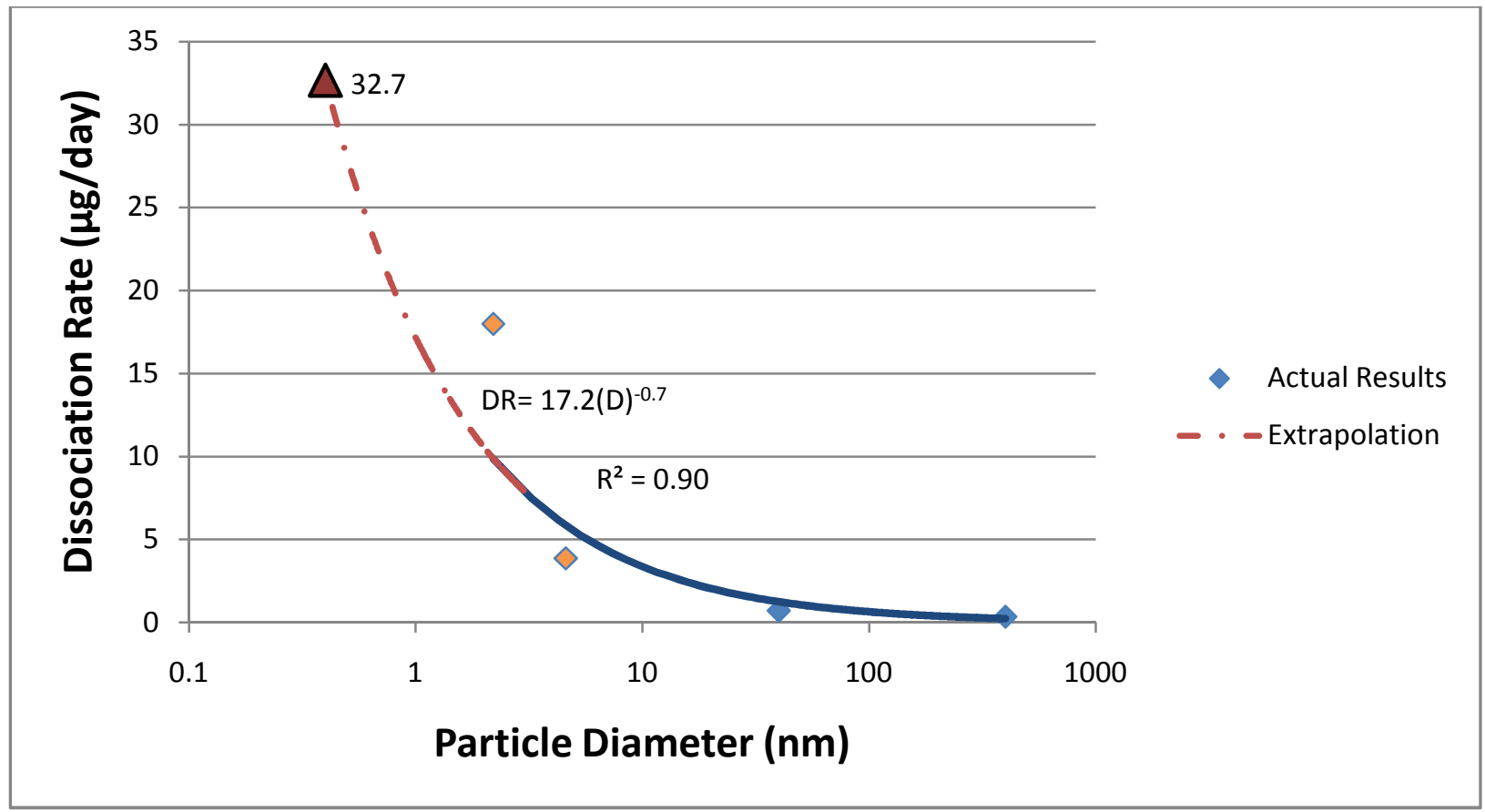

Figure3.25: The power fit curve used to incorporate the data from this experiment (blue points), work by Browne et al. (orange points), and an extrapolation (red line) to true wear debris dimensions (red points). 
In order to put this extrapolated rate in perspective, actual dissolution times for $100 \mathrm{mg}$ of particles at given diameters were estimated using the same data. At these calculated dissolution rates, $100 \mathrm{mg}$ of $400 \mu \mathrm{m}$ diameter spheres would require approximately 26,600 years to completely dissociate into ions, $100 \mathrm{mg}$ of $40 \mu \mathrm{m}$ diameter spheres would require 1,530 years to dissociate, and $0.4 \mu \mathrm{m}$ diameter spheres would completely dissociate into ions within 5.09 years. As stated, these values are provided for perspective only and are based on significant assumptions; however, they do emphasize the dramatic effect of decreasing particle size at the nano-scale.

\section{COMPARING MODEL TO CLINICAL RESULTS}

It is challenging to accurately compare this model to true clinical results, because clinical studies analyze serum and urine samples from patients without filtering wear debris from the samples. This suggests that clinical studies may yield both ion and particulate concentrations, not exclusively metal ion concentrations; however an attempt at comparing the empirical data discussed here with clinical results was made. According to a 2005 in vitro study of ion dissociation from implant materials, ion dissociation was not steady state and upwards of $80 \%$ of net dissociation took place during the first 24 hours of immersion (Figure 3.26). This finding was used as a basis for comparisons between the findings discussed here and clinical results. In evaluating clinical results, the general assumption was made

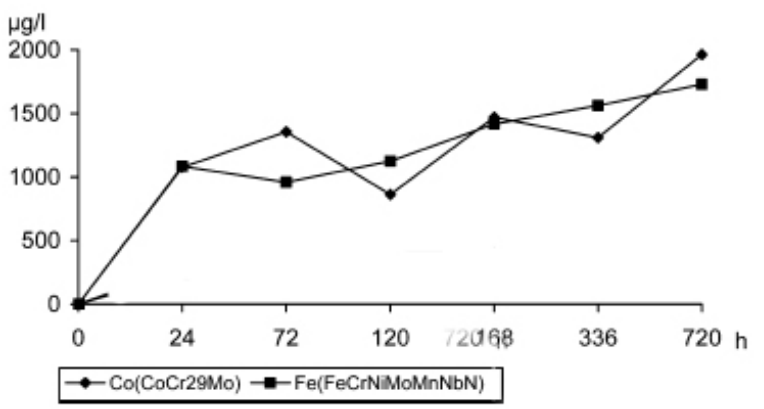

Figure3.26: In vitro serum concentrations of $\mathrm{Co}$ in a wear and dissociation study. Serum ion levels were shown to become relatively saturated after a 24 hour period (Mumme, et al., 2005).

that the entirety of accumulated ions detected in serum were dissociated within 24 hours, 
and the serum ion concentration remained steady state after this period. In addition, it was also assumed for concentration calculations that all patients had the reported average 5.6L of blood (Occupational Safety \& Health Administration, 2008).

Three clinical studies were compared to the results of this thesis (Figure 3.27). The studies all tested patient blood serum for concentrations of Co ions. A 2002 study found patients to have a mean blood serum Co concentration of $1.33 \mathrm{ppm}$ (Savarino, et al., 2002). This was compared to a longer study that found MoM arthroplasty patients to have 8.13 ppm Co ion concentrations (Lhotka, Szekeres, Steffan, Zhuber, \& Zweymiiller, 2003), and a European study that reported a mean serum Co ion concentration of 2.17 ppm (Witzleb, Ziegler, Krummenauer, Neumeister, \& Guenther, 2008). As can be discerned from Figure 3.39, the extrapolated $32.7 \mu \mathrm{g}$ of ions for a $100 \mathrm{mg}$ sample of 400 $\mathrm{nm}$ diameter wear debris would be equivalent to $0.00589 \mathrm{ppm}$ of Co ions in $5.6 \mathrm{~L}$ of blood. Although there are numerous assumptions supporting this conclusion, including steady state blood ion concentrations, $100 \%$ dissolution of wear debris in clinical samples, ions being exclusively in blood, and lack of other confounding factors, this result is remarkably close to the clinical data reported by other researchers in the field and warrants further investigation. 


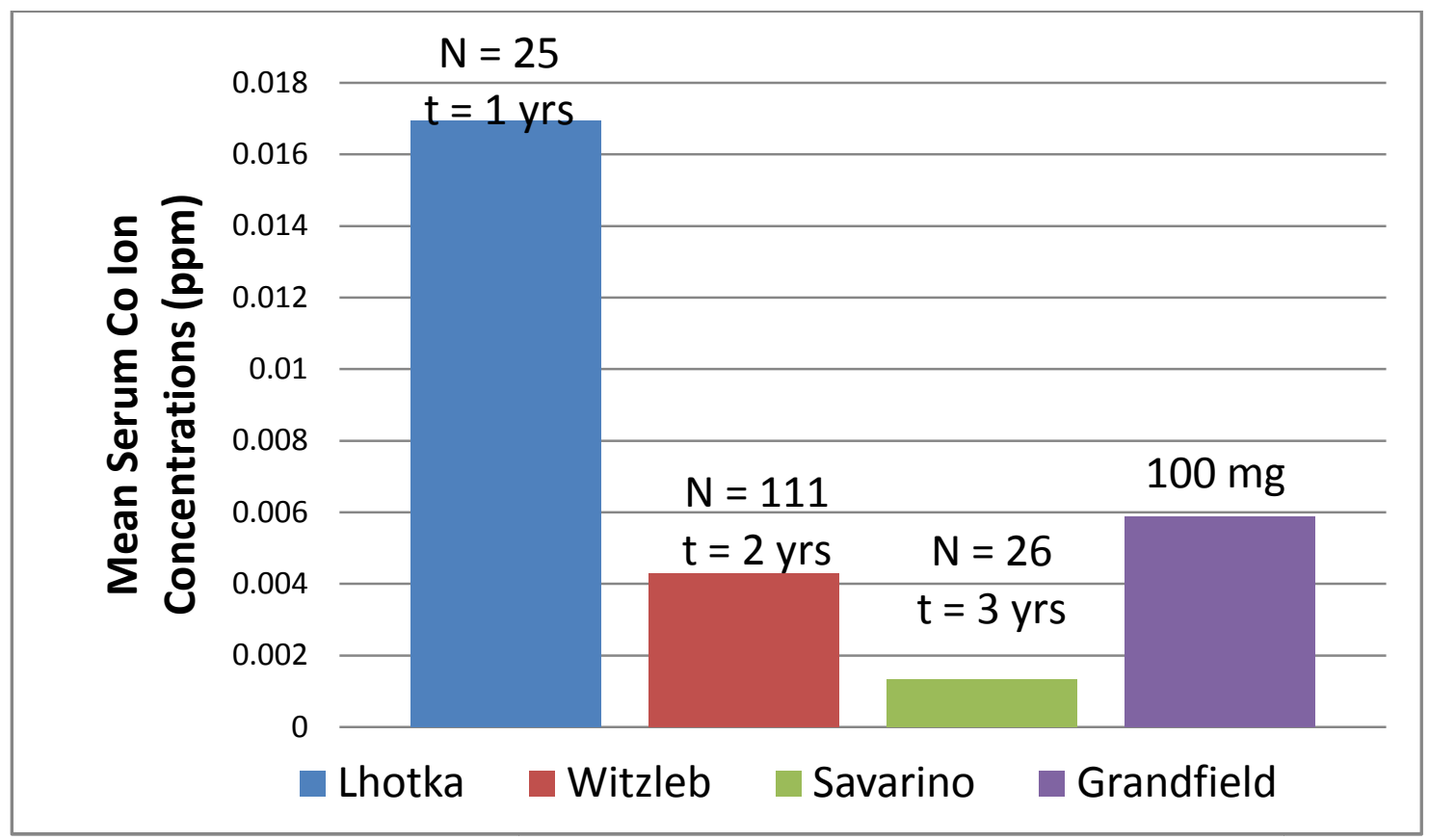

Figure 3.27: Clinical and empirical results for particulate dissociation rates.

\subsection{EVALUATING SURFACE MORPHOLOGIES}

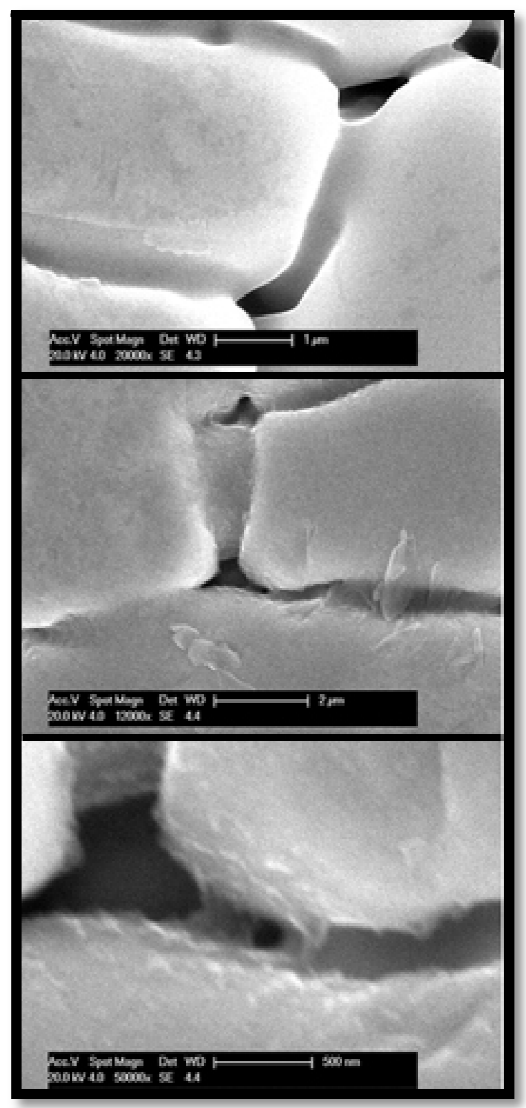

In order to further investigate the corrosion kinetics

of the $\mathrm{CoCr}$ alloy in simulated body conditions, the surface morphologies of both corroded and uncorroded samples were examined using SEM (Figure 3.28), EDS, and FIB. This array of techniques was used to yield the maximum information about sample surfaces as well as to provide supporting evidence to any conclusions drawn using ion concentration data.

Immediately after completion of the ICP ion concentration analysis of the fifth experiment samples, random spheres were collected for each diameter and $\mathrm{pH}$

Figure 3.28: Mild corrosion effects were visible at close magnifications when comparing 
level. The FEI SEM was used to take several images of each sample and comparative visual studies were performed. Although little evidence of corrosion could be seen at low magnifications, closer inspection revealed indications of corrosive attack (Figure 3.28). These indications included general exfoliation and increased surface roughness, suggesting material loss. While these indications were not particularly damaging in nature, they were vast in number and were fairly uniform over the surface of the alloy. This minor corrosion damage confirms the presence of ions in the electrolyte and validates the ICP readings to some degree, although no significant visual difference was identifiable between the samples at $\mathrm{pH} 3$ and $\mathrm{pH}$ 7, nor was a visible difference present between the two sample diameters.

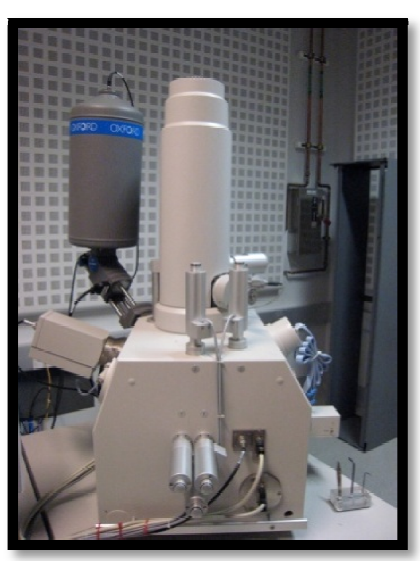

Figure 3.29: The SEM and EDS system used for particle analysis.
The EDS (Figure 3.29) was used to identify compositional variations between sample surfaces exposed to electrolyte. The spectra (Figure 3.30) and resulting compositional data from the various samples were compiled and evaluated for significant differences. Although there were not enough samples to determine statistically significant conclusions, an apparent loss of cobalt content was identified in corroded samples. All corroded specimens showed decreased

cobalt weight percent, which corresponded to

the increasing cobalt concentrations detected

in solution. EDS mapping was used to validate

the highly uniform surface distribution of

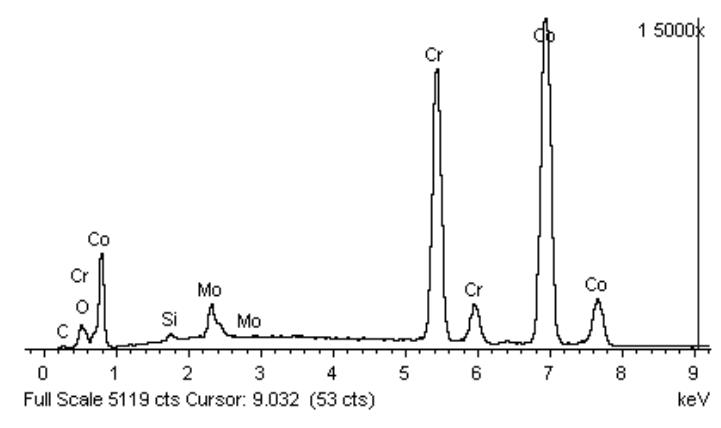

Figure 3.30: Typical EDS spectra for CoCr alloy. 
compositional elements (Figure 3.6). The minor variations in uniformity were represented by slight increases of chromium near grain boundaries, as discussed in section 3.4.2.

To investigate oxide layer formation on the sphere surface, a Focused Ion Beam (FIB) was used to remove material from the surface of various particles in such a way that a cross section of the interior material was visible for analysis. Because the FIB provides an incision using a current of only $200 \mathrm{pA}$ or less, the immediate surrounding material is negligibly affected by the material removal. Initial depth profiles are taken with higher currents and finer, more focused cuts use a less powerful current. The FIB was used to perform subsurface analysis for samples of $40 \mu \mathrm{m}$ diameter sphere specimens placed in $\mathrm{pH} 7$ and $\mathrm{pH} 3$ electrolytes. The depth analysis was intended to detect an oxide layer on the surface of the specimens. Oxide layers of significantly different depths would potentially create the differences in data trends between $\mathrm{pH}$ levels seen in section 3.4.4 (Figure 3.11).

After removing material with the FIB, the cross sectioned regions were imaged using scanning electron (Figure 3.31) and backscatter electron (Figure 3.38) detection in

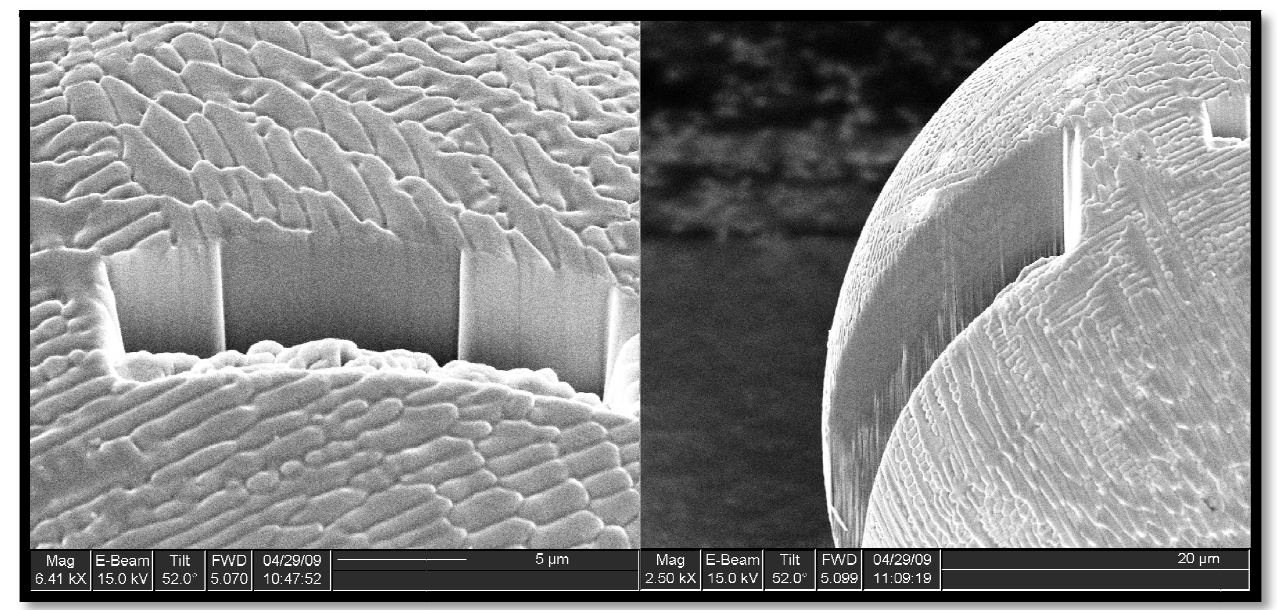

Figure 3.31: FIB cross sections of the CoCr spheres. Cross sections were performed with the intention of characterising a distinctive oxide layer depth. 
the SEM. Although the slight contrast gradient in the backscatter image of the large cross section in Figure 3.32 suggests potential compositional variation along the $\mathrm{z}$ axis, the results required further evidence to validate. EDS analysis was performed on several cross sections in order to acquire a better understanding of the oxide growth characteristics of the CoCr particles. The EDS analysis performed did not confirm the potential oxide layer seen in Figure 3.32 and failed to suggest any distinctive oxide development. While this theory cannot be completely ruled out through this analysis alone, it does present a strong case against an oxide layer causing distinct differences in ion dissociation kinetics at lowered pHs.

Ultimately, the data for cobalt dissociation gathered during this experiment does correlate with the original predictions and reinforces the surface energy theory regarding elevated dissolution rates for smaller wear debris. The data for chromium dissolution

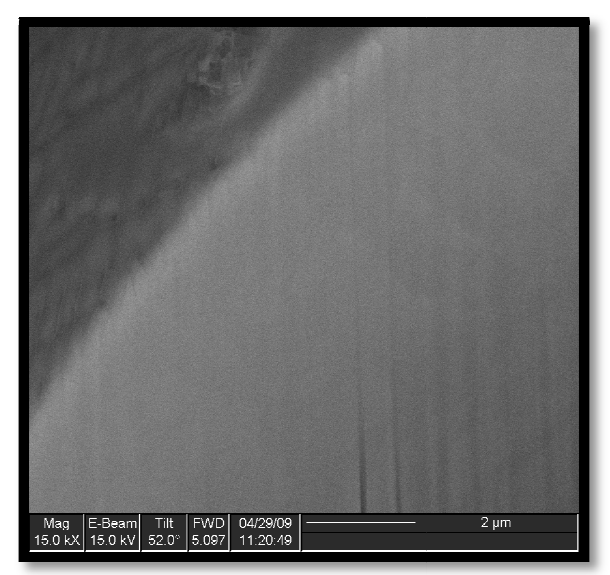

requires further study to validate the methodology used in this experiment. This result supports the case that the consequences of implant wear debris require further analysis prior to a long-term safety determination for patients.

Figure 3.32: Backscatter detection of the FIB cross section seen in figure 4.26. The lighter region near the sample surface potentially indicates a compositional difference and possible oxide layer. 


\section{CONCLUSION}

\subsection{OVERVIEW OF OBJECTIVES}

The intent of this research was to determine the dependence of ion dissociation rate and corrosion kinetics on the surface area to volume ratio of wear debris ejected from bulk MoM hip implants. The completion of this goal required the development and use of an experimental methodology that was validated through the corrosion of a variety of particle sizes, the results of which will be extrapolated to actual implant wear debris. The specific objectives of this research can be summarized in the following four goals:

1) Design, build, and test an apparatus for the quantification of an ion release rate from orthopaedic wear in simulated in vivo conditions.

2) Create an experimental methodology to accurately and repeatably evaluate the ion release rate of a specimen that represents $\mathrm{CoCr}$ wear debris.

3) Analyze data in order to establish an actual ion release rate from representative cobalt chromium alloy particles.

4) Relate the specimen ion release rate to particle surface area to volume ratio.

\subsection{OBJECTIVE 1: APPARATUS CONSTRUCTION}

After refinement, the apparatus constructed for the purpose of simulating in vivo conditions worked well to recreate conditions found in the body. It provided repeatable data that was representative of the targeted bodily conditions, including temperature $\left(37^{0} \mathrm{C}\right), \mathrm{pH}(7)$, and proximity to other wear particles (particle density on the order of $1 \mathrm{x}$ $10^{9}$ spheres/sample) (Hirakawa, Bauer, Stulberg, \& Wilde, 1996). The apparatus was capable of maintaining a uniform temperature of $37^{\circ} \mathrm{C} \pm 1^{0} \mathrm{C}$ throughout the incubated bath which, with the addition of a custom fabricated sample holding system, housed up to 
50 samples. Adding ethylene glycol to the bath water aided in corrosion prevention of the sample holder and a generally cleaner bath environment. In terms of improvements to the apparatus, the only helpful addition would be a larger bath volume in order to hold more samples for tests with higher statistical power.

\subsection{OBJeCtive 2: EXPERIMENTAL METHODOLOGY}

The experimental methodology that was ultimately used in the final experiments produced repeatable results with relatively little error. Error for this experimental methodology was evaluated through standard deviations of separate and independent readings. Standard deviations for Co samples with more than three readings were never greater than $0.0156 \mathrm{ppm}$, and averaged less than $15 \%$ of the total reported ion concentration for the Co samples. Also, the hypothetical prediction of the dissolution rate was shown to be accurate for the smaller debris within an order of magnitude, which reinforces the accuracy of the calculated ion dissociation rate for the simulated wear debris.

The initial sample containment methods involved copious amounts of cleaning and preparation, while yielding results that were potentially contaminated and out of statistical control. Due to the minute ion concentrations of interest to this experiment and the significant possibility of data corruption due to accidental error, the single use of sterile and disposable lab supplies for the storage and testing of samples proved to be the ideal method for maintaining a testing environment with minimal error potential. Unless required, as in the case of the Erlenmeyer flasks during vacuum filtration, the electrolyte samples never made physical contact with a container that was previously used. In the case of the Erlenmeyer flasks, extensive care was taken to guarantee the cleanliness prior 
to each sample extraction. The final data for Cobalt ions, which were the only statistically significant results, proved to agree with the original modeling of the dissociation rate and showed strong trends regarding the surface area dependence of the ion dissociation rate.

\subsection{OBJECTIVE 3: DATA ANALYSIS}

Data analysis was performed using multiple redundantly supportive techniques: both AAS and ICP analysis systems were used in the detection of sample ion concentrations, the SEM was utilized for visual corrosion confirmation, the EDS yielded compositional data, and the FIB allowed for compositional depth examination.

Particularly the use of the ICP allowed for considerable accuracy and repeatability in data collection. While the AAS continuously showed variability in the ion concentrations detected, the ICP was precise and accurate when detecting calibration solutions, consistently yielding results within $1 \%$ of the target value with a standard deviation of less than $0.00152 \mathrm{ppm}$. The ICP also showed consistent results when samples were tested multiple times on different days. The accuracy and repeatability of ion measurements were confirmed primarily through EDS investigation. The EDS provides compositional data for samples and was consistent in detecting results that correlated strongly with the ICP ion concentrations.

\subsection{Objective 4: Correlation Between Surface Area to Volume RATIO AND DISSOCIATION RATE}

As discussed in Chapter 3, the successful collection of statistically significant data that supported both the prediction detailed in section 3.5.2. and understanding of particle dissociation kinetics required numerous revisions in the experimental methodology as well as the detection methods. Ultimately, the revised methodology produced data that was measurable when compared to prior work and clinical trials. The 
methodology repeatability was defined by the statistical significance of the data in relation to controls, the calibration of the detection systems, and the reinforcing data retrieved from further analysis of the wear debris itself.

\subsubsection{COBALT DISSOLUTION}

The surface area to volume dependent dissociation rate for cobalt ions from 100 mg of $40 \mu \mathrm{m}$ diameter spheres of ASTM F75 CoCr in pH 7 HBSS was determined to be $0.0111 \mu \mathrm{g}$ ions/day. Confirming the original comparative hypothesis of surface area to volume dependent corrosion rates, the dissociation rate for cobalt ions from a $400 \mu \mathrm{m}$ diameter ASTM F75 CoCr sphere in pH 7 HBSS was $0.00625 \mu \mathrm{g}$ ions/day. These results were expected to be lower than the $0.772 \mu \mathrm{g}$ ions/day hypothesized for the $40 \mu \mathrm{m}$ sphere samples and the $0.359 \mu \mathrm{g}$ ions/day predicted for the $400 \mu \mathrm{m}$ spheres.

\subsubsection{CHROMIUM DISSOLUTION}

The dissociation rates for chromium ions from $100 \mathrm{mg}$ of $40 \mu \mathrm{m}$ and $400 \mu \mathrm{m}$ diameter spheres of ASTM F75 CoCr in pH 7 HBSS were determined to be $0.000222 \mu \mathrm{g}$ ions/day and $0.000251 \mu \mathrm{g}$ ions/day, respectively. Although these results do not coincide with the results for cobalt, the detected values of chromium at $\mathrm{pH} 7$ were on the order of $0.001 \mathrm{ppm}$ and were not considered statistically different from the control results, particularly because the theoretical detection limit for chromium in the ICP is $0.01 \mathrm{ppm}$. With this information, the dissociation rate of chromium at $\mathrm{pH} 7$ was determined to be too slow for this exploratory study, and chromium results were discarded.

\subsubsection{DISCUSSION OF PH MODIFICATIONS}

Also of note, the ion concentration results for $\mathrm{pH} 3$ solutions were significantly higher than the samples immersed in $\mathrm{pH} 7$ solutions, however the samples with $\mathrm{CoCr}$ densities greater than $1 \mathrm{~g} / \mathrm{L}$ of HBSS may have been altered by agglomeration problems, 
as discussed in section 3.4.4. These results, while statistically significant, do not relate well to the human body and were used exclusively for informational purposes.

\subsection{CONCLUDING STATEMENT}

The overall experimental results support the conclusion that, particularly for cobalt, which, in contrast to some literature, was found to be the primary dissociation species in $\mathrm{pH} 7 \mathrm{HBSS}$, the rate of ion dissociation appears to be dependent on total surface area to volume ratio, which increases with decreasing particle diameter; thus the rate of Co ion dissociation is related to particle diameter (Figure 4.1).

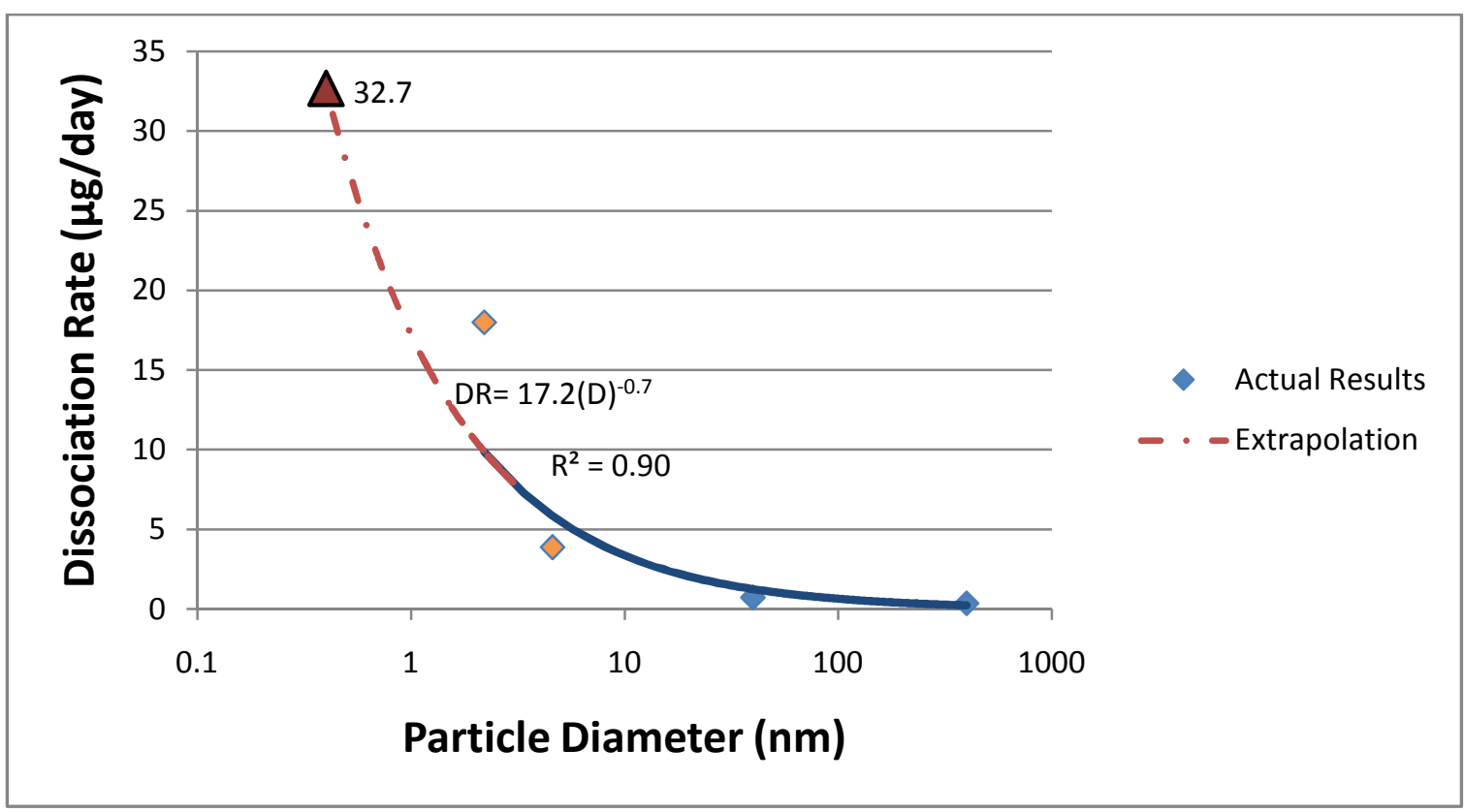

Figure 4.1: Extrapolation of exponential fit curve for empirical data to true wear debris diameters. The blue points indicate experimental data from this experiment, while the red data point is a suggested dissociation rate for true wear debris of $400 \mathrm{~nm}$ diameter.This extrapolation (red point) emphasizes the exponential relationship between total particle surface area to volume ratio and decreasing particle diameter.

These results are relevant to true wear debris, as true MoM wear debris is on the order of $0.4 \mu \mathrm{m}$ in diameter and would likely have exponentially greater surface energies and thus exponentially higher ion dissociation rates. The data yielded in this experiment was extrapolated using a power fit in order to yield an estimated dissolution rate of 32.7 
$\mu \mathrm{g}$ ions/day for $0.4 \mu \mathrm{m}$ diameter debris, significantly higher than that of the $40 \mu \mathrm{m}$ simulated wear debris tested in this study. These high dissociation rates have the potential for significant long term consequences in the human body. 


\section{FUTURE WORK}

There are numerous ways in which the ion dissociation kinetics of systemically distributed wear debris may potentially be further evaluated, although four are particularly pertinent to this study: (1) the advancement of the study to true wear debris (Figure 5.1), (2) the use of extended time duration, (3) periodic rate studies to identify dissociation rate changes that may occur throughout the immersion process over time, (4)

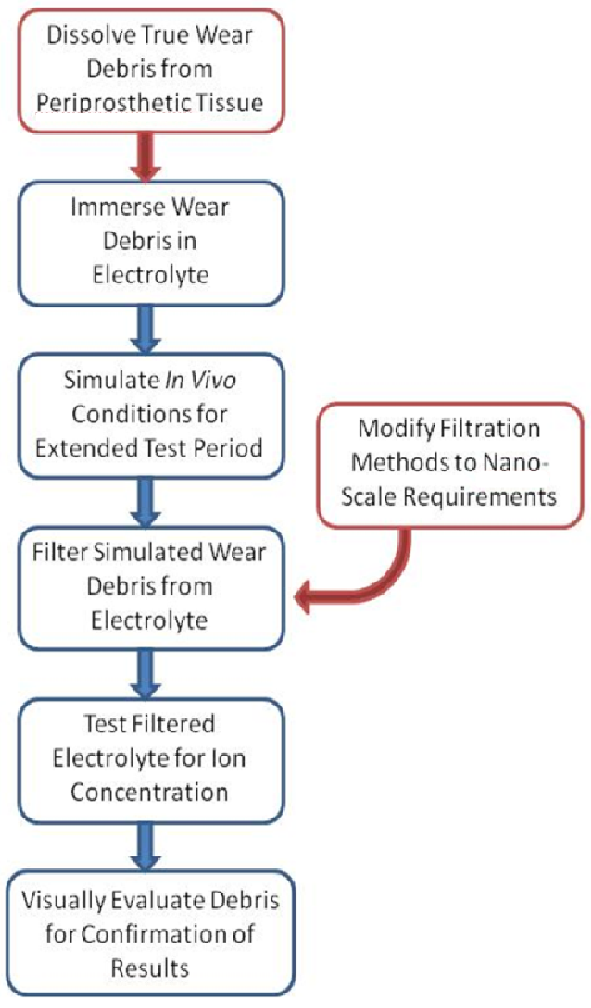

Figure 5.1: The simplified procedure (blue) used for this series of tests and the addition of necessary steps and improvements (red) for the application of this test methodology to true wear debris. and further studies of the specific ion species that are released during the course of particle dissolution.

The true intent of this research was the development and validation of a test methodology capable of accurately determining ion disassociation kinetics for nano-scale particles. With the successful collection of data that indicates findings consistent with modern surface energy phenomena, the test methodology is currently applicable to true implant wear debris with several minor modifications. The most difficult steps in analyzing true wear debris are the collection of the wear debris itself and filtering the debris from the electrolyte after immersion. The

suggested method for the collection of MoM implant wear debris is the utilization of recently explanted metallosis tissue, which is tissue proximal to the implant that has been saturated with metal wear debris (Figure 5.2). The metallosis tissue provides a valuable 
source of true metal wear debris in an easily accessible location and can potentially provide enough debris to complete a high-powered statistical study of the dissociation rates of small particle groupings.

The debris to be tested for ion dissociation rate must be separated from the tissue in which it is embedded, collected, and cleaned of any dissolving agents prior to immersion in the electrolyte. The methodology used in this paper can then be used with a modified filtration method. The filtration method used in this testing utilized $2 \mu \mathrm{m}$ pore filters, through which a vacuum system pulled the electrolyte after the test period. The simulated wear debris was large enough to be completely isolated by the filter, leaving pure electrolyte with only ions to be tested. However, due to the varied size and inconsistent diameter of true wear debris, a significantly smaller filter pore size would be

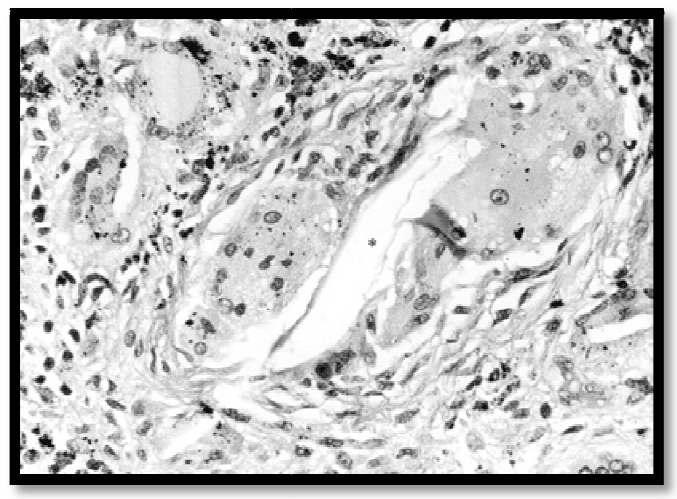

Figure 5.2: Photomicrograph of synovial tissue with large amount of particulate metal, giant cells, and reactive fibrosis. (Hematoxylin-eosin stain,) (original magnification x 400) (McGovern \& Moskal, 2002)

required to completely isolate the electrolyte from the debris to ensure accurate testing, which would thus require a significant increase in vacuum strength and system rigidity. As an alternative to greatly increasing the cost of the vacuum filtration system to meet the stringent demands of completely isolating true wear nano-scale debris, it is recommended that an ultracentrifuge be used to separate components by density. Due to the drastic difference in metal and electrolyte densities, it is likely that there would be no crossover of constituents, leading to a completely pure and ideal electrolyte for analysis.

Implementing the use of true wear debris in the methodology used for this testing would 
greatly increase the relevance of the data collected with regard to applicability to implants in the human body.

In addition to upgrading the experimental methodology to be capable of testing true isolated wear debris, an important advancement of this study would be a broader study analyzing and comparing a wider variety of particle diameters in a test with dramatically extended time duration and implementing periodic testing of ion concentrations throughout the experiment. Because it is unlikely that ion dissociation from the particle surface is constant, periodic analysis would provide a perspective on the time dependence of the ion dissociation rate and the dependence of dissociation rate on oxide formation. This periodic analysis would likely introduce new difficulties in accounting for electrolyte removed for destructive testing, although it would introduce a great deal of valuable understanding to the field.

Lastly, further research into quantitative characterization of the specific ion species released by the wear debris during the dissolution process could prove relevant in advancing an understanding of precisely how implant wear debris interacts in the body. Because neither the ICP nor the AAS yield ion specific data, it is unknown which actual ions leave the surface of the debris. This is significant because, while still toxic, $\mathrm{Cr}^{3+}$ is less damaging to the human body than $\mathrm{Cr}^{6+}$ (Safety and Health Topics: Hexavalent Chromium, 23). Additionally, ions that leave the unoxidized surface of CoCr alloy can change their valence state during travel through the oxide layer on the surface of the alloy. Because this change in valence state is mass transport dependent and can occur multiple times, information about the ions released from a pure, unoxidized $\mathrm{CoCr}$ surface is not truly applicable to the in vivo ion release characteristics. A quantitative evaluation 
of which species of ions ultimately leave the surface of the wear debris would be a difficult, albeit valuable, addition to modern in vivo debris dissolution science. 


\section{WORKS CiTED}

Baslé, M., Bertrand, G., Guyetant, S., Chappard, D., \& Lesourd, M. (1996). Migration of metal and polyethylene particles from articular prostheses may generate lymphadenopathy with histiocytosis. Journal of biomedical materials research , 30, 157 164.

Brown, C., Williams, S., Tipper, J. L., Fisher, J., \& Ingham, E. (2006). Characterisation of wear particles produced by metal on metal and ceramic on metal hip prostheses under standard and microseparation simulation. Journal of materials science. Materials in medicine, 18, 819-827.

Browne, M., \& Gregson, P. (1995). Metal ion release from wear particles produced by Ti-6Al4V and Co-Cr alloy surfaces articulating against bone. Materials Letters , 1-6.

Brune, D., Hultquist, G., \& Leygraf, C. (1984; 92). Corrosion resistance of a passivated and non-passivated cobalt-chromium alloy. Scand J Dent Res , 262-7.

Campbell, P., Amstutz, H., Kossovsky, N., \& Clarke, I. (1992). Mechanism and clinical significance of wear debris-induced osteolysis. Clin Orthop Relat Res. (276), 7-18.

Carr, B. C., \& Goswami, T. (2007). Knee implants - Review of models and biomechanics. Materials \& Design, 398-413 .

Catelas, I., Campbell, P., Bobyn, J., Medley, J., \& Huk, O. (2006). Wear particles from metal-on-metal total hip replacements: effects of implant design and implantation time. Proceedings of the Institution of Mechanical Engineers, Part H: Journal of Engineering in Medicine , 220 (2), 195-208.

Catelas, I., Medley, J. B., Campbell, P. A., Huk, O. L., \& Bobyn, J. D. (2004).

Comparison of In Vitro with In Vivo Characteristics of Wear Particles from Metal-Metal Hip Implants. Journal of biomedical materials research, 70B, 167-178.

Chan, F. W., Bobyn, D. J., Medley, J. B., Krygier, J. J., \& Tanzer, a. M. (1999). Wear and lubrication of metal-on-metal implants. Clinical orthopaedics and related research (369), 10-24.

Chidambaram, R., \& Cobb, A. (2007). Change in the Age Distribution of Patients Undergoing Primary Hip and Knee Replacements Over 13 Years. Journal of Bone and Joint Surgery - British Volume , Vol 91-B (152), 152.

Cuckler, J. (2005). The rationale for metal-on-metal total hip arthroplasty. Clin Orthop Relat Res (441), 132-136. 
DeFrances, C. J., \& Hall, M. J. (2007). 2005 National Hospital Discharge Survey. Atlanta, GA: Center for Disease Control and Prevention.

Direct Healthcare International. (2008). Hip Replacement Surgery. (Direct Healthcare International) Retrieved 3 15, 2009, from http://www.directhealthcare.com/hip_replacement.htm

Dowson, D. (2006). Tribological principles in metal-on-metal hip joint design. Journal of Engineering in Medicine, 161-171.

Dumbleton, J., \& Manley, M. (2005). Metal-on-Metal total hip replacement: what does the literature say? Joint Arthroplasty, 20, 822-823.

Dunn, H. K., Peters, C. L., \& Hofmann, A. A. (2000). Hip Replacement. (The University of Utah) Retrieved 4 4, 2009, from University Healthcare:

http://healthcare.utah.edu/orthopaedics/patients/education/hipreplacement.html

Eremenko, V. (1959). The Role of Surface Phenomena in Metallurgy. New York, New York: Consultants Bureau.

Gladstone, S. L. (1941). The Theory of Rate Processes. New York: McGraw-Hill.

Granchi, D., Cenni, E., Ciapetti, G., Savarino, L., Stea, S., Gamberini, S., et al. (1998).

Cell death induced by metal ions: Necrosis or apoptosis? J Mater Sci Mater Med, 31-37.

Gray, R. (1974). A History of the Haynes Stellite Company. Cabot Corporation.

Hallab, N., Skipor, A., \& Jacobs, J. J. (2003). Interfacial kinetics of titanium- and cobaltbased implant alloys in human serum. Journal of biomedical materials research, 311318.

Herberts, P., Kärrholm, J., \& Garellick, G. (2004). Annual Report. Sahlgrenska: Swedish National Hip Arthoplasty Register.

Hip Anatomy. (2008, 10 14). (Zimmer, Inc.) Retrieved 4 12, 2009, from Zimmer United States: http://www.zimmer.com/z/ctl/op/global/action/1/id/389/template/PC/navid/164

Hirakawa, Bauer, T. W., Stulberg, B. N., \& Wilde, A. H. (1996). Comparison and quantitation of wear debris of failed total hip and total knee arthroplasty. Journal of biomedical materials research, 31 (2), 257-263.

Hirakawa, Bauer, T., Stulberg, B., Wilde, S., \& Secic, M. (1996). Caracterization and Comparison of Wear Debris from Failed Total Hip Implants of Diferent Types. The Journal of Bone and Joint Surgery, 78-A (8), 1235-1243. 
Hsu, C.-C. Y.-A.-S. (2005). Electrochemical corrosion studies on Co-Cr-Mo implant alloy in biological solutions. Materials Chemistry and Physics , 531 - 538.

Isabelle, C., Medley, J., Campbell, P., Huk, O., \& Bobyn, D. (2004). Comparison of In Vitro with In Vivo Characteristics of Wear Particles from Metal-Metal Hip Implants. Journal of Biomedical Materials Research , 167-178.

Jakobsen, S. S., Danscher, G., Stoltenberg, M., Larsen, A., Bruun, J. M., Mygind, T., et al. (2007). Cobalt-Chromium-Molybdenum Alloy Causes Metal Accumulation and Metallothionein Up-Regulation in Rat Liver and Kidney. Basic \& Clinical Pharmacology \& Toxicology , 441-446.

Krokslätts, F. (2007, November). Arcam EBM material information. Retrieved November 30, 2008, from Arcam EBM System: www.arcam.com

Kurtz, S. M. (2004). The UHMWPE handbook. San Diego, Ca: Elsevier Academic Press.

Lee, H., Brennan, F. R., Jacobs, J. J., Urban, R. M., Ragasa, D. R., \& Glant, T. T. (1997). Human Monocyte/Macrophage Response to Cobalt-Chromium Corrosion Products and Titanium Particles in Patients with Total Joint Replacements. Journal of Orthopaedic Research , 40-49.

Lee, J. M., Salvati, E. A., Betts, F., DiCarlo, E. F., Doty, S. B., \& Bullough, a. P. (1992). Size of metallic and polyethylene debris particles in failed cemented total hip replacement. Journal of Bone and Joint Surgery - British Volume , 74-B (3), 380-384.

Lhotka, C., Szekeres, T., Steffan, I., Zhuber, K., \& Zweymiiller, K. (2003). Four-year study of cobalt and chromium blood levels in patients managed with two different metalon-metal total hip implants. Journal of Orthopaedic Research , 189-195.

Liaoa, Y., McKellopa, H., Lua, Z., Campbell, P., \& Benyaa, P. (2003). The effect of frictional heating and forced cooling on the serum lubricant and wear of UHMW polyethylene cups against cobalt-chromium and zirconia balls. Biomaterials 24,3047 3059.

Lin, H.-Y., \& Bumgardner, J. D. (2004). In vitro biocorrosion of Co-Cr-Mo implant alloy by macrophage cells. Journal of Orthopaedic Research , 22, 1231-1236.

Liu, T.-K., Liu, S.-H., Chang, C.-H., Yang, \& Rong-Sen, a. (1998). Concentration of Metal Elements in the Blood and Urine in the Patients with Cementless Total Knee Arthroplasty. Journal of Experimental Medicine , 253-262.

MacDonald, S., McCalden, R., Chess, D., Bourne, R., Rorabeck, C., Cleland, D., et al. (2003). Metal-on-metal versus polyethylene in hip arthroplasty: a randomized clinical trial. Clinical orthopaedics and related research, 406, 282-296. 
MacRitchie, F. (1990). Chemistry at Interfaces. San Diego, California: Academic Press, Inc.

McGovern, T. F., \& Moskal, J. T. (2002). Radiographic Evaluation of Periprosthetic Metallosis: Materials and Methods. J South Orthop Assoc. 2002 , 1-11.

Merritt, K., \& Brown, S. (1996). Distribution of Cobalt Chromium Wear and Corrosion Products and Biologic Reactions. Clinical Orthopaedics and Related Research, 223-243.

Metikos-Hukovic, M., \& Babic, R. (2007). Passivation and corrosion behaviours of cobalt and cobalt-chromium-molybdenum alloy. Corrosion Science , 3570-3579.

Michel, R., Noite, M., Reich, M., \& Loer, F. (1991). Systemic effects of implanted prostheses. Arch Orthop Trauma Surg. , 61-74.

Midander, J. P. (2006). Elaboration of a test method for the study of metal release from stainless steel particles in artificial biological media. Corrosion Science, 2855-2866.

Milose, I., Trebse, R., Kovac, S., Cor, A., \& Campbell, P. (2005). Dissociation of the metal inlay from the polyethylene liner in an uncemented threaded cup. Orthopaedic Trauma Surgery, 134-141.

Mumme, T., Muller-Rath, R., Jakobi, N., Weißkopf, M., Dott, W., Marx, R., et al. (2005). In vitro serum levels of metal ions released from orthopaedic implants. Eur J Orthop Surg Traumatology, 83-89.

Naudie, D., Roeder, C., Parvizi, J., Berry, D., Eggli, S., \& Busato, A. (2009). Metal-onmetal versus metal-on-polyethylene bearings in total hip arthroplasty: A matched casecontrol study. The Journal of Arthroplasty , 19 (7), 35-41.

Occupational Safety \& Health Administration. (2008, May 23). United States Department of Labor. Retrieved Jan 9, 2009, from Safety and Health Topics: Hexavalent Chromium: http://www.osha.gov/SLTC/hexavalentchromium/index.html

Osborn, J. J. (1953). Experimental Hypothermia: Respiratory and Blood ph Changes in Relation to Cardiac Function. Am J Physiol , 389 - 398.

Papageorgiou, I., Yin, Z., Ladon, D., Baird, D., Lewis, A. C., Sood, A., et al. (2007). Genotoxic effects of particles of surgical cobalt chrome alloy on human cells of different age in vitro. Mutation Research , 45-58.

Posner, A., Anderson, J., \& Alexander, A. (1954). Colloidal and Surface Phenomena. Industrial Engineering Chemistry, 898-916.

Quesnel, T., Gueritey, P., \& Gonon, a. G. (1995). Biomechanics of the hip: forces exerted during walking. Surgical and Radiologic Anatomy (17), 249-253. 
Rae, T. (1981). The toxicity of metals used in orthopaedic prostheses: An experimental study using cultured human synovial fibroblasts. J Bone Joint Surg Br, 435-440.

Registry, A. f. (2001). ToxFAQs: Chromium. Centers for Disease Control and Prevention.

Safety and Health Topics: Hexavalent Chromium. (23, May 2008). Retrieved Mar 2009, 16, from United States Department of Labor: Occupational Safety \& Health

Administration: http://www.osha.gov/SLTC/hexavalentchromium/index.html

Savarino, L., Granchi, D., Ciapetti, G., Cenni, E., Greco, M., Rotini, R., et al. (2002). Ion release in stable hip arthroplasties using metal-on-metal articulating surfaces: A comparison between short and medium-term results. Journal of Biomedical materials Research , 450-456.

Savarino, L., Granchi, D., Ciapetti, G., Cenni, E., Pantoli, A. N., Rotini, R., et al. (2002). Ion Release in Patients with Metal-on-Metal Hip Bearings in Total Joint Replacement. Journal of biomedical materials research , 63, 467-474.

Savarino, L., Stea, S., Granchi, D., Visentin, M., Ciapetti, G., Donati, M., et al. (2000). Sister chromatid exchanges and ion release in patients. Journal of biomedical materials research (50), 21-26.

Surin, V. (2009, 1 1). Metal-on-Metal Total Hips. Retrieved 4 14, 2009, from Facts About Total Joints: http://www.totaljoints.info/metal_on_metal_total_hips.htm

Virtanen, S., Kurz-Hochstrasser, S., \& Hodgson, A. (2001). Passivity and Corrosion Behavior of Co-28Cr-6Mo Implant Alloy. Institute of Materials Chemistry and Corrosion, 1.

Von Knoch, D. E. (2004). The effectiveness of polyethylene versus titanium particles in inducing osteolysis in vivo. Journal of Orthopaedic Research 22 , 237-243.

Willert, G., Buchhorn, H., Gobel, D., Koster, G., Schafner, S., Schenk, R., et al. (1996). Wear behavior and histopathology of classic cemented metal on metal hip endoprostheses. Clinical orthopaedics and related research (329), 160-186.

Williams, J. A. (2005). Engineering Tribology. Cambridge: Cambridge University Press.

Wimmer, A., Nassutt, R., Sprecher, C., Loos, J., Täger, G., \& Fischer, A. (2006). Investigation on stick phenomena in metal-on-metal hip joints after resting periods. Proc Inst Mech Eng [H] , 220, 219-227.

Witzleb, W.-C., Ziegler, J., Krummenauer, F., Neumeister, V., \& Guenther, K.-P. (2008). Exposure to chromium, cobalt and molybdenum from metal-on-metal total hip replacement and hip resurfacing arthroplasty. Acta Orthopaedica , 697-705. 
